\title{
LINEWIDTH AND RANGING CHARACTERIZATION OF A VERNIER-TUNED DISTRIBUTED BRAGG REFLECTOR (VT-DBR) ALL-SEMICONDUCTOR TUNABLE SWEPT LASER SYSTEM FOR LIDAR IN AUTONOMOUS APPLICATIONS
}

\author{
A Thesis \\ presented to \\ the Faculty of California Polytechnic State University, \\ San Luis Obispo
}

\author{
In Partial Fulfillment \\ of the Requirements for the Degree \\ Master of Science in Electrical Engineering
}

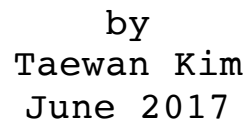


(c) 2017

Taewan Kim

ALL RIGHTS RESERVED 
COMMITTEE MEMBERSHIP

TITLE: Linewidth and Ranging Characterization of a VernierTuned Distributed Bragg Reflector (VT-DBR) All-Semiconductor Tunable Swept Laser System for Lidar in Autonomous Applications

AUTHOR: Taewan Kim

DATE SUBMITTED: June 2017

\begin{abstract}
COMMITTEE CHAIR: Dennis Derickson, Ph.D. Professor of Electrical Engineering
\end{abstract}

COMMITTEE MEMBER: Xiaomin Jin, Ph.D. Professor of Electrical Engineering

COMMITTEE MEMBER: Vladimir Prodanov, Ph.D. Associate Professor of Electrical Engineering 


\section{ABSTRACT}

Linewidth and Ranging Characterization of a Vernier-Tuned Distributed Bragg Reflector (VT-DBR) All-Semiconductor Tunable Swept Laser System for Lidar in Autonomous Applications

Taewan Kim

Linewidth and ranging experiments of a Insight packaged Vernier-Tuned Distributed Bragg Reflector (VT-DBR) laser across its wavelength output range of 1522.13 to $1566.18 \mathrm{~nm}$ is done in this work to characterize it for lidar applications. The purpose of this paper is to investigate the laser's potential to combine the advantages of lidar and FMCW radar for autonomous systems.

Linewidth measurements were done by using a MachZehnder interferometer to set up a delayed self-homodyne measurement. The laser was set to output at a fixed wavelength across a range of 1523 to $1566 \mathrm{~nm}$ in $1 \mathrm{~nm}$ increments, and linewidth was captured each of these increments. For each of the linewidths, coherence time and length along with laser currents were associated. The minimum linewidth found in this test was found to be 50 $\mathrm{MHz}$, leading to a maximum coherence time of $6.366 \mathrm{~ns}$ and a maximum coherence length of $129.92 \mathrm{~cm}$. There was a somewhat linear, albeit low correlation, area of low linewidths depending on the front mirror and back mirror currents across the wavelength range.

Initial ranging experiments were performed using interference fringes caused by variable stationary path length differences introduced into a homemade Mach-Zehnder interferometer around the coherence lengths found in the linewidth test. The experimental path length differences indicated by the interference fringes seem to be accurate at very small ruler measured path length differences, but starts to stray away from the ruler measured as the path length differences get larger. Data taken suggests that there is a mathematical relationship in the error between the ruler measured and experimental path length differences leading to the belief that this error can be compensated for.

Keywords: Vernier-Tuned Distributed Bragg Reflector, VTDBR, FMCW, linewidth, coherence, self-homodyne, MachZehnder Interferometer, interference fringes 


\section{ACKNOWLEDGMENTS}

This project was made possible due to the guidance of Professor Dennis Derickson. I am grateful for his willingness to answer all questions I had about the theory and technicalities regarding optics and for making himself available while I was working on the experiments. Another thank you to Chris Wood and Jason Ensher of Insight Photonics Solutions for providing the laser hardware and their willingness to answer questions along with their relay of helpful info through various phone calls and email exchanges.

Finally, I would like to thank Professor Xiaomin Jin and Professor Vladimir Prodanov for participating on my thesis committee. 
TABLE OF CONTENTS

Page

LIST OF TABLES • • • • • • • • • • • • • • • • • • • • • • • •

LIST OF FIGURES • • • • • • • • • • • • • • • • • • • • • • ix

\section{CHAPTER}

1. Introduction. • • • • • • • • • • • • • • • • • • • • 1

1.1 Example of Autonomy Technology: Driverless Cars. 1

1.2 Frequency Modulated Continuous-Wave (FMCW) • • • • 5

1.3 Swept Laser in Optical Coherence Tomography (OCT) 11

1.4 Purpose of Study • • • • • • • • • • • • • • • 13

1.5 Thesis Summary. • • • • • • • • • • • • • • 16

2. The Insight VT-DBR Laser and Linewidth. • • • • • • • 17

2.1 Laser Components and the VT-DBR Laser. • • • • • 17

2.2 Capabilities of Insight Laser. • • • • • • • • . 21

2.3 Linewidth and Coherence. . . . . . . . • • . 23

3. Laser Linewidth Characterization. • • • • • • • • • • 27

3.1 Laser Linewidth Test Setup and Theory. • • • • • 27

3.2 Laser Linewidth Test. • • • • • • • • • • • 32 
3.3 Laser Linewidth Test Results and Analysis. • • .

4. Ranging with Interference Fringes. . . . . . . . . 53

4.1 Interference Fringe Test Setup and Theory. . . . 53

4.2 Interference Fringe Test. . . . . . . . . . 64

4.3 Interference Fringe Test Calibration Method . . 69

4.4 Interference Fringe Test Results and Analysis. . 72

5. Conclusions. . . . . . . . . . . . . . 78

5.1 Insight Laser Linewidth. . . . . . . . . . 79

5.2 Ranging with Insight Laser. . . . . . . . 83

5.3 Future Work. . . . . . . . . . . . 86

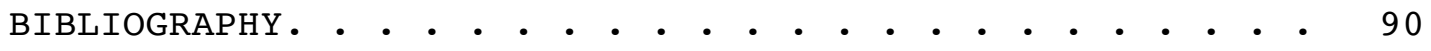

APPENDICES

A. Captured Laser Linewidths. • . . . . . . . 92

B. Laser Lineshapes. . . . . . . . . . . 106

C. Time Domain Interference Fringes. . . . . . . . 111

D. Frequency Domain Interference Fringes. . . . . 114 


\section{LIST OF TABLES}

Table

Page

1. Goals of FMCW lidar sensor and comparison of FMCW method vs conventional time of flight method. . . . . .

2. Comparison of VT-DBR Laser for OCT VS FMCW Lidar. • . 15

3. Peaks in linewidth found at various wavelength outputs of laser. . . . . . . . . . . . . . . . . . .

4. Troughs in linewidth found at various wavelength outputs of laser. . . . . . . . . . . . . . . .

5. Peaks in linewidth found at various wavelength outputs of laser with their corresponding laser currents. . . .

6. Troughs in linewidth found at various wavelength outputs of laser with their corresponding laser currents.

7. Comparison of ruler measured $\Delta L$ and experimental $\Delta L$ (calculated fron interference fringes frequency) for various path lengths. . . . . . . . . . . . . .

8. Goals of the FMCW Lidar sensor within various parameters. • • • • • • • • • • • • • •

9. The increase in error as the ruler measured path length $\Delta L$ difference increases. . . . . . . . . . . . . 84 


\section{LIST OF FIGURES}

Figure

Page

1-1 - A high-level overview of the hardware used on the Google Autonomous Car [4] • • . . • . . . • . • . . . 3

1-2 - The concept of FMCW with a linear sweep of frequencies transmitted [11] • • • • • • • • • • • • • • 66

1-3 - The concept of FMCW with the Doppler effect showing a linear increase and decrease sweep of frequencies transmitted [11] • • • • • • • • • • • • • • • • • • • • 99

1-4 - Ambulance caused Doppler effect with an approached and departed observer [12] • • • • • • • • • • • • • • • 10

1-5 - Biomedical imaging techniques and the unique niche of OCT [13] • • • • • • • • • • • • • • • • • • • • • • 12

1-6 - A typical SSOCT test setup with a sampling output going to the photodetector and two sample arms, one of which is a reference with a fixed mirror [13] • • • • • • 12

2-1 - A conventional laser configuration of a gain medium, pump, and optical resonator consisting of a fully reflective mirror and partially reflective mirror [13] • .

2-2 - The 5 control currents of the VT-DBR to control wavelength tuning [18] • • • • • • • • • • • • • • • 20

2-3 - Front Panel of the Insight Akinetic Laser source [20] • • • • • • • • • • • • • • • • • • • • • • • • • • 21 
2-4 - 5105 Acquisition program with time domain waveform data and waveforms of laser outputs including "Data Valid" and "Start Sweep". . . . . . . . . . . . . . 22

2-5 - Interference fringe test setup for a ruler measured path length difference of $1 \mathrm{~cm}$. . . . . . . . . . 22

2-6 - Typical Lorentzian-shaped central peak laser

spectrum with relaxation oscillations and sidemodes [21]. 24

2-7 - Concept of coherence time with an example of (a) coherent light, and (b) short coherence [21]. • . . . 25

3-1 - Insight Laser Control Program fixed wavelength output interface $[20] \cdot$. . . . . . . . . . . . 28

3-2 - Fixed $1540 \mathrm{~nm}$ output of laser shown in optical spectrum analyzer. . . . . . . . . . . . . . 29

3-3 - Mach-Zehnder, Micheson, and Fabry interferometers seen in delayed self-homodyne measurement setups [21] • • 31

3-4 - (a) Laser lineshape mixing with (b) delayed self for (c) output electrical spectrum shape [21]. • . . . . . . . 32

3-5 - Insight laser delayed self-homodyne experimental test setup: laser is output into interferometer for mixing with delayed self into photodetector to observe output spectrum in electrical spectrum analyzer. . . . . . . . 33

3-6 - Example of experimental data taken for fixed wavelength output of $1523 \mathrm{~nm} \cdot$. . . . . . . . . . . 35

3-7 - Theoretical Lorentzian spectral shape at measured points of $-3 \mathrm{~dB},-10 \mathrm{~dB},-20 \mathrm{~dB}$, and $-30 \mathrm{~dB}[21]$. . . . 36 
3-8 - Example of experimental data recorded of width at various $d B$ down from peak for fixed wavelength output of $1523 \mathrm{~nm}$. . . . . . . . . . . . . . . . . 36

3-9 - Laser linewidth graphed against fixed wavelength laser output. . . . . . . . . . . . . . . . . 39

3-10 - Peak linewidths across the 1523 to $1566 \mathrm{~nm}$ wavelength range under test. . . . . . . . . . . . 40

3-11 - Trough linewidths across the 1523 to $1566 \mathrm{~nm}$ wavelength range under test. . . . . . . . . . . . . 41

3-12 - (a) Front mirror (fm), (b) back mirror (bm), (c)

phase, and (d) semiconductor optical amplifier (soa)

currents variability across the wavelength output range. • 43

3-13 - Front mirror (fm) and back mirror (bm) currents at linewidth peaks across the tested wavelength range. . . . 46

3-14 - Trend of the front mirror (fm) and back mirror (bm) currents at linewidth troughs across the tested wavelength range. . . . . . . . . . . . . . . . . . . . 47

3-15 - Trough front mirror (trough fm) and peak front mirror (peak fm) currents plotted on same graph to highlight deviation from proposed trend. . . . . . . . 48

3-16 - Trough back mirror (trough bm) and peak back mirror (peak bm) currents plotted on same graph to highlight deviation from proposed trend. . . . . . . . . . . . 49 
3-17 - Frequencies at $3 \mathrm{~dB}(\Delta v), 10 \mathrm{~dB}\left(\Delta v_{10 \mathrm{~dB}}\right), 20 \mathrm{~dB}$

$\left(\Delta v_{20 d B}\right)$, and $30 \mathrm{~dB}\left(\Delta v_{30 d B}\right)$ down from peak for wavelengths

across the range of 1523 to $1566 \mathrm{~nm}$. . . . . . . . . 51

3-18 - Lorentzian spectral shape and a Gaussian spectral

shape overlaid [23]. . . . . . . . . . . . . . 52

4-1 - Insight Laser Control Program swept wavelength

output interface $[20]$. . . . . . . . . . . 54

4-2 - Swept $1522.13 \mathrm{~nm}$ to $1566.18 \mathrm{~nm}$ output of laser shown

in optical spectrum analyzer. . . . . . . . . . . 55

4-3 - The delta wavelength of the sweep $\Delta \lambda$ is the

difference in the sweep of the laser between two peaks of

the interference fringes. . . . . . . . . . . 57

4-4 - Example of time domain interference fringes at (a) a

small $\Delta L$ (b) a medium $\Delta L$ (c) a large $\Delta L$. . . . . . . . . 59

4-5 - Phase and length relationship across two different

lengths. . . . . . . . . . . . . . . . . . 60

4-6 - Homemade Mach-Zehnder interferometer allowing for

interchangeable lengths. . . . . . . . . . . . 63

4-7 - Homemade two paddle polarization controller. . . . 64

4-8 - Time domain interference fringe test setup. . . . 65

4-9 - Interference fringes as seen on 5105 Acquisition

program across a time scale of around 3 laser sweeps for a

ruler measured path length difference of $<1 \mathrm{~cm}$. . . . . 66 
4-10 - Example of experimental data taken for ruler measured path length difference of $<1 \mathrm{~cm}$ in time domain interference fringe test. . . . . . . . . . . . . . .

4-11 - Frequency domain interference fringe test setup. .

4-12 - Example of experimental data taken for ruler measured path length difference of $<1 \mathrm{~cm}$ in frequency domain interference fringe test. . . . . . . . . . 69

4-13 - Time domain output of calibration interferometer with known $100 \mathrm{pm} \Delta \lambda$. . . . . . . . . . . . . . . . . . 71

4-14 - Frequency domain output of calibration interferometer with known $100 \mathrm{pm} \Delta \lambda$. . . . . . . . . . 71

4-15 - Comparison of ruler measured $\Delta L$ and experimental $\Delta L$ for various path length measurements. . . . . . . . . . 74

4-16 - Barely discernible interference fringes at a ruler measured path length difference of $130.5 \mathrm{~cm}$. . . . • . 76

4-17 - Barely discernible frequency of interference fringes at a ruler measured path length difference of $130.5 \mathrm{~cm}$. . . . . . . . . . . . . . . .

4-18 - Indiscernible interference fringes at a ruler measured path length difference of $187.5 \mathrm{~cm}$. . . . . . 77 4-19 - Indiscernible frequency of interference fringes at a ruler measured path length difference of $187.5 \mathrm{~cm}$. . . 77 
5-1 - Low linewidth front mirror (trough $\mathrm{fm}$ ) and high

linewidth front mirror (peak fm) currents plotted on same

graph to highlight deviation of high linewidth currents

from proposed trend of low linewidth currents. . . . . . 81

5-2 - Low linewidth back mirror (trough bm) and high

linewidth back mirror (peak bm) currents plotted on same

graph to highlight deviation of high linewidth currents

from proposed trend of low linewidth currents. . . . . . 82

5-3 - Difference between the ruler measured path length differences and experimental path length differences as the ruler measured path length difference increases from

Measurement Number 1 to 9. . . . . . . . . . . . 85 


\section{Chapter 1. Introduction}

With the onset of a more technologically focused future, the ability of everyday items to be "smart" is in demand more so than ever before. What is exactly a "smart" item? Something that can learn, something that can do tasks for a person, but all in all, something that further satisfies the human desire to do less in the pursuit of a "comfortable" future in which human involvement is minimized but productivity of that said person is the same or improved. In this pursuit of "smart" items or "comfortability" there is the drive for development of fully autonomous vehicles.

From a very high level, an autonomous vehicle can be defined as a vehicle that can drive or fly itself. Further delving into this concept, the vehicle would have to be able to safely transport itself from Point A to Point B with efficiency, comfortability, safety, and everything in between while completing tasks that it may be given. From drones to planes, rovers to cars, an automated future is in demand.

\subsection{Example of Autonomy Technology: Driverless Cars}

In the world today, especially in tech hubs such as Silicon Valley, driverless cars in particular are of huge interest. When presented with this challenge that can 
rebound with large profits, top companies such as Google, Uber, and Tesla initiated research and development for driverless tech in the hopes of being the first company to create a common consumer purposed fully autonomous car. These companies all have different methods of approach for their goals. For example, Google has the autonomous car development as part of their Research and Development sector of the company called Waymo [1], Uber has bought out many faculty and facilities of Carnegie Mellon University to give them a head start [2], and Tesla is now producing cars that have full autonomous hardware in order to gather data from everyday drivers as they continue to develop the autonomous software needed for full autonomy in parallel [3] •

Although all of these companies are in constant software development to develop the artificial intelligence needed for full autonomy, they seem to have convened on the hardware necessary for their software to be loaded unto. In other words, the hardware system necessary to receive data from the surroundings of said autonomous car and needed for actuation of received data has a commonality between all of these cars hoping to be self-driving. To provide a better understanding of this system, the hardware in this system will be listed and the use of each will be described. 
Figure 1-1 highlights the hardware that is installed on Google's self-driving cars along with the costs involved for each piece.

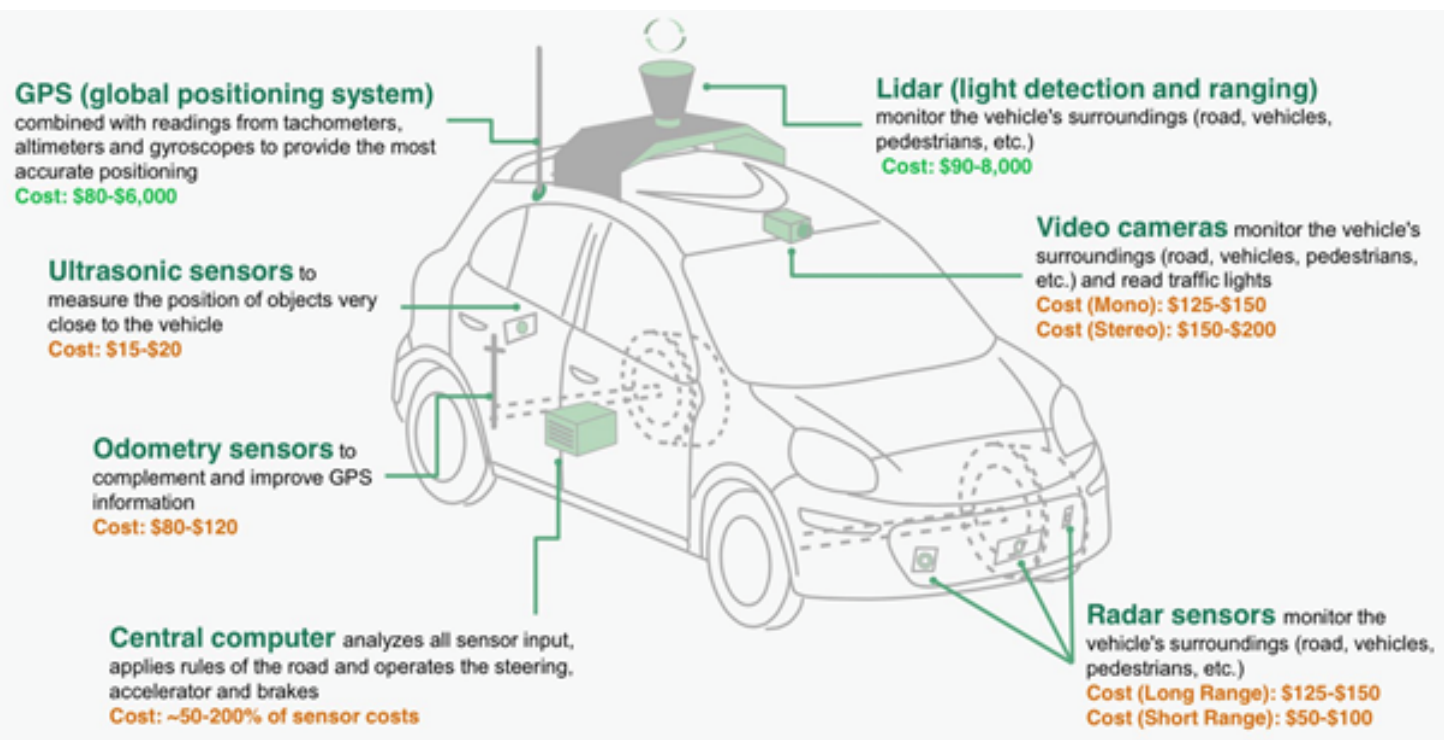

Figure 1-1 - A high-level overview of the hardware used on the Google Autonomous Car [4].

Observing what has been revealed by Google, the different types of sensors in terms of hardware needed for a selfdriving car are GPS, Ultrasonic Sensor, Odometry Sensor, Lidar, and Radar Sensor.

GPS, Short for Global Positioning System is used for location identification. The problem with GPS becomes resolution. GPS with the current technology available today for the consumer and from a business analytics point of view the most affordable GPS tech in terms of return on investment is meter length resolution. Only very expensive versions allow centimeter accuracy, though the push towards 
innovation for less expensive highly accurate GPS is in the works [5] .

Ultrasonic sensors are used for distance measurement and is composed of a transmitter and receiver and transmits a high frequency sound pulse and measures the amount of time it takes for that pulse to be received back after being reflected from another object to be able to estimate the distance between the ultrasonic sensor and said object [6] •

Odometry Sensors are used to measure change in position over time. An example of how this might work is having rotary encoders on a wheel which will indicate how far the wheels have rotated, and knowing the wheel circumference, distance traveled can be found [7].

Lidar uses a surveying method used for object detection by illuminating said object with a laser light. An array of detectors or a timed camera can read in light reflections from the short pulses of light emitted, to create a 3D point "cloud" for vector information and volume identification [8] •

Radar sensors, which are commonly frequency-modulated continuous-wave (FMCW) radar, is composed of a transmitter and receiver and uses $\mathrm{RF}$ technology to determine speed, proximity, range, and object size. Both lidar and radar are 
used commonly used in autonomous systems to cross validate what they're seeing and to predict motion [9].

With the many technologies available for the purpose of an autonomous vehicle briefly outlined, it would be of great interest to be able to explore technology that is able to combine the benefits of a two into a new sensor. Given that radar and lidar are commonly used to cross validate, if there was a method of utilizing a laser and its benefits of angular accuracy along with the benefits of the FMCW method of radar, it could be extremely beneficial to the arsenal of hardware that may be needed for autonomous systems in the future.

\subsection{Frequency Modulated Continuous-Wave (FMCW)}

Ordinary pulsed radar detects an object by emitting a short pulse and calculating the time of flight of the reflected pulse off the object. This practice of using short pulses requires the radar to have a high instantaneous transmit power which results in a larger and more expensive physical apparatus for the radar [10]. Frequency Modulated Continuous-Wave (FMCW) radars, on the other hand, can achieve similar results as ordinary pulsed radars but with a lesser instantaneous transmit power and thus physical apparatus by using continuously emitting continuous pulses whose frequencies vary over time [10]. 
Further, the continuous wave sweep nature of FMCW allows it to detect velocity as well, while ordinary pulsed radars cannot continuously monitor and as a result, cannot accurately measure velocity of a detected object. Called a linear FM sweep, the range to the object of interest is found by detecting the frequency difference between the transmitted and received signals. As such, the range between the radar and the object is proportional to the difference in frequencies (called the beat frequency) [11].
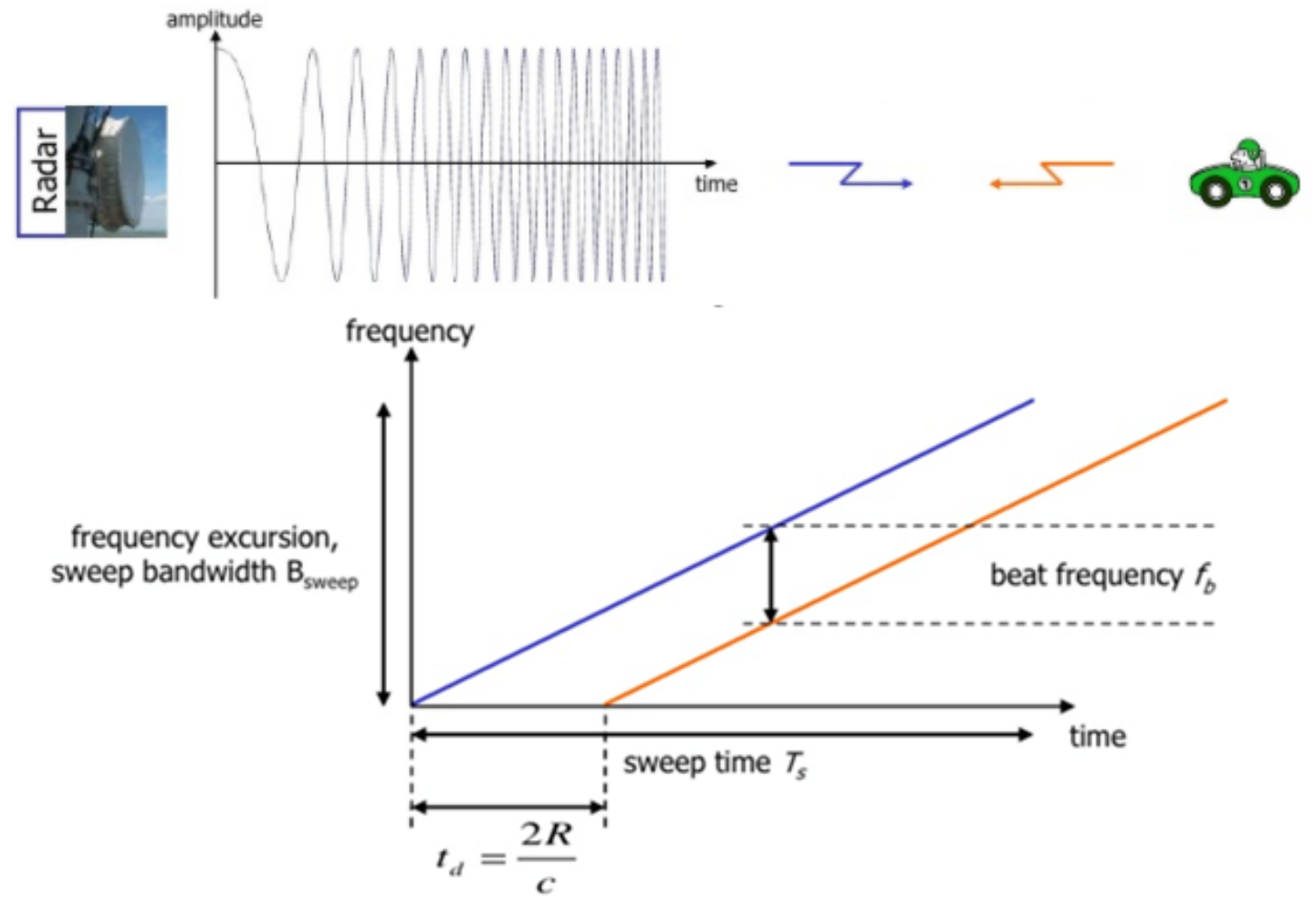

Figure 1-2 - The concept of FMCW with a linear sweep of frequencies transmitted [11].

Figure 1-2 provides a visual representation of FMCW. As illustrated, the transmitter of the radar sends out 
pulses of linearly increasing frequencies over time and this repeats at a rate commonly called the sweep rate. These pulses are reflected off the object of interest and the receiver receives a frequency which is indicative of the round-trip delay as shown which is proportional to the distance of the object from the radar.

Further explaining, how this would work is that with each repetition of the frequency sweeps (governed by the sweep rate), there is a range of frequencies (governed by the sweep bandwidth) that is sent out. Following those frequencies to the object of detection, they will reflect off the object, and return to the receiver of the radar. As these frequencies return to the receiver, the difference in frequencies transmitted vs. received is called the beat frequency and can be correlated with a difference in time called the delay time. With this frequency and time difference data received and utilizing the speed of light, the distance to the object can be calculated. In equation form, the relationship between all these factors that determine the distance between the radar and the object is shown as follows [11]:

$$
\begin{gathered}
\frac{t_{d}}{T_{s}}=\frac{f_{b}}{B_{\text {sweep }}} \\
R=\frac{c T_{s} f_{b}}{2 * B_{\text {sweep }}}
\end{gathered}
$$




$$
\begin{aligned}
& \text { where: } \\
& c=\text { speed of light } \\
& t_{d}=\text { delay time }(s) \\
& f_{b}=\text { beat frequency }(\mathrm{Hz}) \\
& R=\text { distance between antenna and the reflecting object }(m) \\
& T_{s}=\text { sweep time }(s) \\
& B_{\text {sweep }}=\text { sweep bandwidth }(\mathrm{Hz})
\end{aligned}
$$
object. 

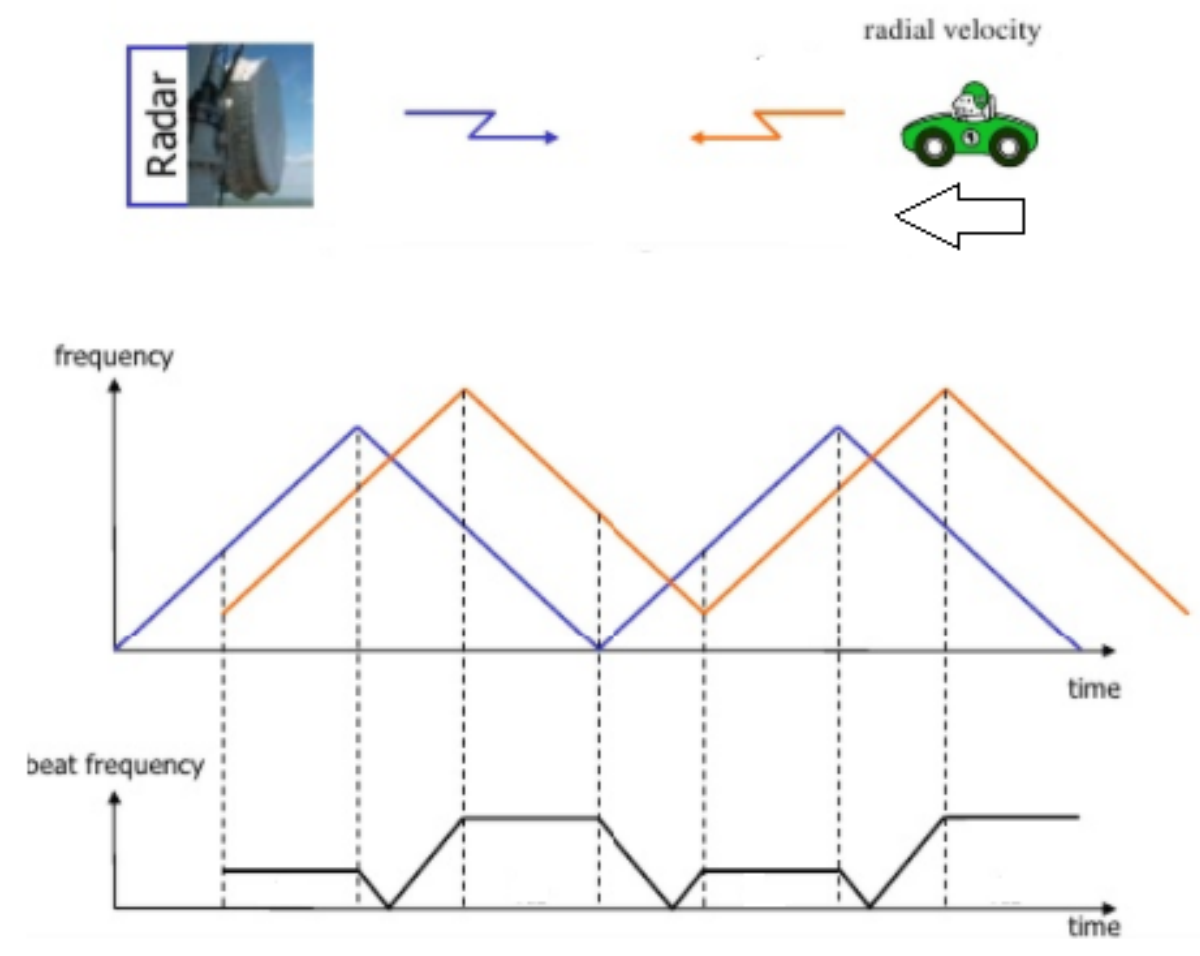

Figure 1-3 - The concept of FMCW with the Doppler effect showing a linear increase and decrease sweep of frequencies transmitted [11].

Figure 1-3 provides a visual representation of FMCW with the Doppler effect. As illustrated, the transmitter of the radar outputs a linear increase and decrease of frequencies, and as they reflect off the object of interest approaching the radar, the beat frequency is higher or lower depending on the increasing or decreasing sweep of frequencies received at the receiver.

The Doppler effect can be explained further at a high level using a simple example. If one is an observer to an ambulance that is emitting its siren sound at a certain constant frequency, and that ambulance is approaching the 
observer, the frequency of the sound to the approached observer is higher than the true emitted frequency of the moving ambulance. However, on the flip side, to an observer that the ambulance is departing from, the frequency of the sound is lower than the true emitted frequency. A visual representation of this can be found in Figure 1-4.

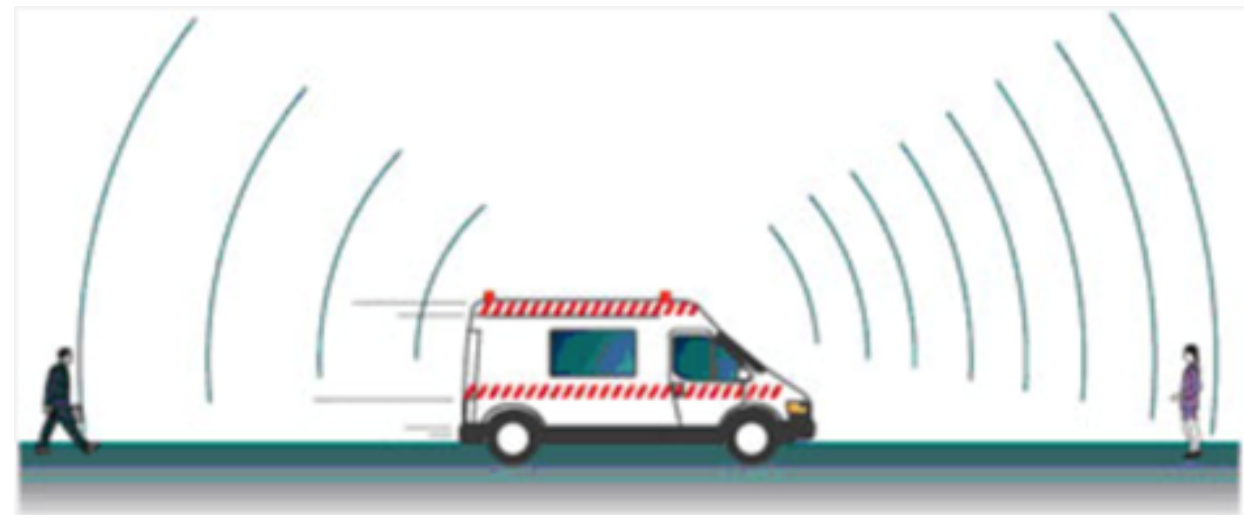

Figure 1-4 - Ambulance caused Doppler effect with an approached and departed observer [12].

Along with the simple concept of ranging both still and moving objects with the FMCW method, comes another benefit, which is security from jamming. With autonomous systems reliant on their sensors as much as humans are reliant on their senses to move around, it is extremely important that the sensors are resistant to those who may want to trick them into sensing something false, bringing them offline, or worse, causing an accident. Because in FMCW, the frequency is constantly changing, it stands as a formidable system to trick. In order for one to jam the radar, they would first have to know the operating range of 
frequencies (sweep bandwidth) and operate within that range so that a receiver can acknowledge the false frequency as within its bandwidth. Furthermore, the jammer would also have to know the step frequency (slope of the linear increase) of the signal transmitted by the radar it is trying to jam and would have to successfully emulate such a signal. Compared to another sensor such as lidar, where there is simply a short pulse of light that is sent out and received, FMCW is much more difficult to emulate, and as a result, jam.

\subsection{Swept Laser in Optical Coherence Tomography (OCT)}

OCT is an imaging technique used for translucent and partially opaque materials first demonstrated in the early 1990s [13]. It is commonly used for biomedical imaging in cardiology, optometry, dermatology, and early cancer detection where it can offer sub-micrometer isotropic resolution images [13]. OCT can be seen as a optical ultrasound where highly coherent light instead of sound is used. OCT can be performed in or ex vivo, as shown in Figure 1-5 allowing a dynamic imaging modality that is minimally invasive and can offer high resolution imaging [13] 


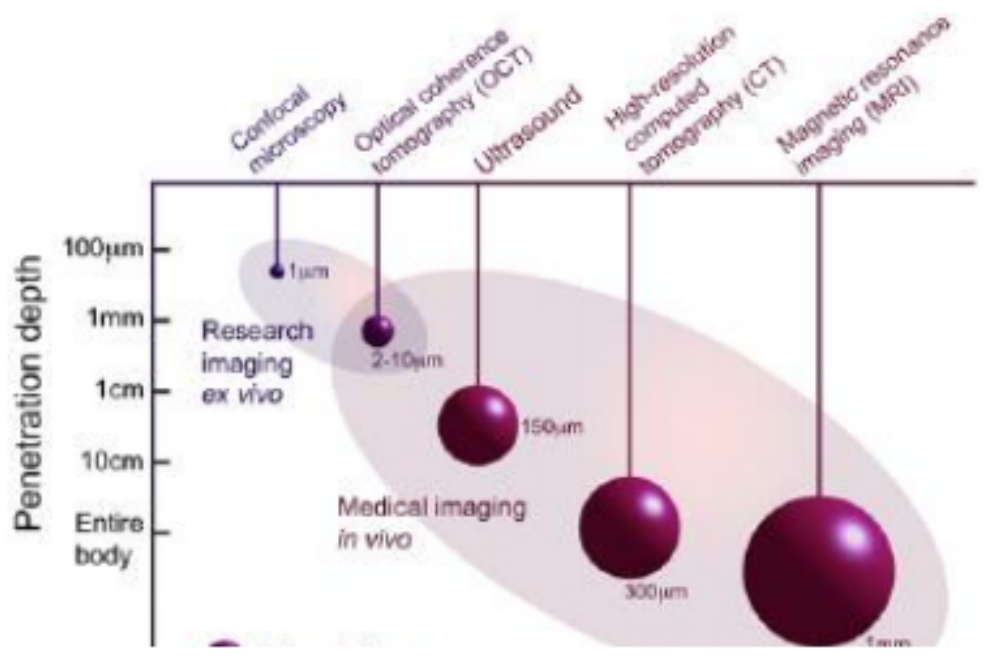

Figure 1-5 - Biomedical imaging techniques and the unique niche of OCT [13].

Recent developments of OCT have brought upon several different methods with unique advantages, and among them, there in source-swept OCT (SSOCT). In SSOCT, a broadband swept light source is required [13]. Figure 1-6 illustrates a high-level block diagram of an SSOCT system.

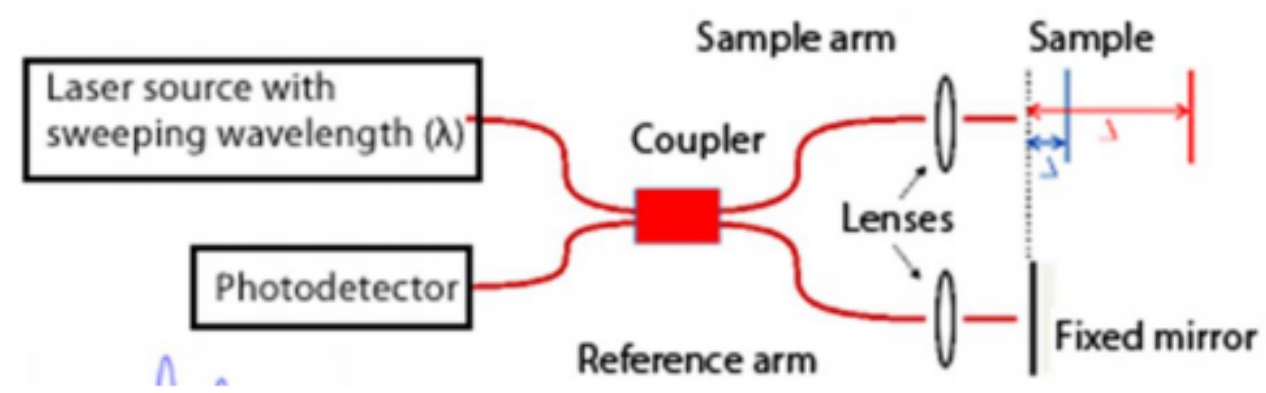

Figure 1-6 - A typical SSOCT test setup with a sampling output going to the photodetector and two sample arms, one of which is a reference with a fixed mirror [13]. 
Figure 1-6 is implemented as a fiber-coupled Michelson interferometer and the sample arm is used for imaging something such as an artery, tooth surface, or skin [13]. This method, also know as Fourier Domain OCT (FDOCT) has been shown to have a sensitivity advantage over traditional time domain ОСт [13]. Swept frequency lasers are an ideal source for this approach and have continually demonstrated success .

\subsection{Purpose of Study}

This study uses an Insight Photonics packaged VernierTuned Distributed Bragg Reflector (VT-DBR) which is an allsemiconductor, high-speed, tunable laser. Tunable meaning that the wavelength can be controlled over a certain range [13]. As such, just like how FMCW radar sweeps across a range of frequencies, this can be done with the tunable laser to sweep across its range of wavelengths. The VT-DBR so far has demonstrated success in achieving highresolution images of the human body in OCT applications [13] •

The purpose of this study is to investigate into the feasibility of using this laser for ranging purposes similar to how the FMCW method works for radar in order to combine the benefits of both a lidar and radar into one FMCW lidar sensor. The overall goals of such a sensor and 
the potential benefits of FMCW lidar over conventional time of flight lidar is summarized in Table 1 .

Table 1 - Goals of FMCW lidar sensor and comparison of FMCW method vs conventional time of flight method.

\begin{tabular}{|c|c|c|c|c|}
\hline Parameter & $\begin{array}{l}\text { Goal } \\
\text { Value }\end{array}$ & Comments & $\begin{array}{c}\text { FMCW } \\
\text { Specific }\end{array}$ & $\begin{array}{l}\text { Time of } \\
\text { Flight } \\
\text { Specific }\end{array}$ \\
\hline Range/Sensitivity & $100 \mathrm{~m}$ & $\begin{array}{l}\text { Should be } \\
\text { able to sense } \\
\text { something } \\
\text { like a stop } \\
\text { sign at } 100 \mathrm{~m} \\
\text { with adequate } \\
\text { signal to } \\
\text { noise ratio. }\end{array}$ & $\begin{array}{l}\text { Heterodyne } \\
\text { detection } \\
\text { is used. }\end{array}$ & $\begin{array}{l}\text { Requires } \\
\text { Geiger- } \\
\text { Mode } \\
\text { detectors. }\end{array}$ \\
\hline $\begin{array}{l}\text { Distance } \\
\text { Resolution }\end{array}$ & $10 \mathrm{~cm}$ & $\mathrm{~N} / \mathrm{A}$ & $\begin{array}{l}\text { Requires } \\
\text { sweep } \\
\text { bandwidth } \\
B_{\text {sweep of } 5} \\
\text { GHz }\end{array}$ & $\begin{array}{l}t=\frac{d}{v} \\
=\frac{10 \mathrm{~cm}}{3 \times 10^{8} \mathrm{~m} / \mathrm{s}} \\
\approx 333 \mathrm{ps}\end{array}$ \\
\hline $\begin{array}{l}\text { Time to take a } \\
\text { single data } \\
\text { point. }\end{array}$ & $2 \mu s$ & $\begin{array}{l}\text { Corresponds } \\
\text { to taking } 500 \\
\mathrm{kSamples/ \textrm {s }}\end{array}$ & $\mathrm{N} / \mathrm{A}$ & $\mathrm{N} / \mathrm{A}$ \\
\hline $\begin{array}{l}\text { Security of } \\
\text { system against } \\
\text { other lidars. }\end{array}$ & TBD & $\begin{array}{l}\text { Must be able } \\
\text { to work in an } \\
\text { environment } \\
\text { with many } \\
\text { simultaneous } \\
\text { LIDARs in } \\
\text { operation, } \\
\text { with little } \\
\text { interference. }\end{array}$ & Robust & $\begin{array}{l}\text { Vulnerable } \\
\text { (easier to } \\
\text { jam with } \\
\text { pulses or } \\
\text { CW) }\end{array}$ \\
\hline $\begin{array}{l}\text { Security against } \\
\text { intentional } \\
\text { jamming. }\end{array}$ & TBD & $\begin{array}{l}\text { Must still } \\
\text { work even } \\
\text { with } \\
\text { intentional } \\
\text { laser jamming } \\
\text { signal. }\end{array}$ & Robust & $\begin{array}{l}\text { Vulnerable } \\
\text { (easier to } \\
\text { jam with } \\
\text { pulses or } \\
\text { CW) }\end{array}$ \\
\hline Cost & TBD & $\begin{array}{l}\text { Minimal part } \\
\text { of autonomous } \\
\text { system cost }\end{array}$ & TBD & TBD \\
\hline Eye Safety & $\begin{array}{l}\text { IEC } \\
\text { Class } 1\end{array}$ & $\begin{array}{l}\text { Class } 1 \\
\text { lasers are } \\
\text { very low risk } \\
\text { and "safe } \\
\text { under } \\
\text { reasonably } \\
\text { foreseeable } \\
\text { use" [14]. }\end{array}$ & $\mathrm{N} / \mathrm{A}$ & $\mathrm{N} / \mathrm{A}$ \\
\hline
\end{tabular}


The extent to which this FMCW lidar application of the VT-DBR differs from a typical OCT application is summarized in Table 2 .

Table 2 - Comparison of VT-DBR Laser for OCT vs FMCW Lidar.

\begin{tabular}{|l|c|c|c|}
\hline \multicolumn{1}{|c|}{ Parameter } & OCT & FMCW Lidar & Comments \\
\hline Tunability Range & $5000 \mathrm{GHz}$ & $\frac{5 \mathrm{GHz}}{2 \mu \mathrm{s}}$ \\
\hline Sweep Rate & $\frac{5000 \mathrm{GHz}}{10 \mu \mathrm{s}}$ & $100 \mathrm{~m}$ \\
\hline Distance Resolution & $10 \mu \mathrm{m}$ & $\begin{array}{l}\text { (Subject of this } \\
\text { thesis) }\end{array}$ \\
\hline Range & $1-2 \mathrm{~cm}$ & Eosy \\
\hline
\end{tabular}

An example of factors that come into play to test the potential success of an FMCW laser and reaching the goal of a range/sensitivity of $100 \mathrm{~m}$ as shown in Table 1 and Table 2 is the range of wavelengths chosen to be swept over. Depending on how the laser is tuned, some wavelengths may yield to lower spectral width than others leading to longer or more accurate ranging measurements. Also from there, extracting the theoretical maximum range of the laser would be useful to determine what applications the current configuration of the Insight VT-DBR laser would be most fitted for.

Another example of factors that come into play is how accurate the laser ranging is when an object is in its theoretical range. Is the resolution error large? Table 1 indicates a desired distance resolution of $10 \mathrm{~cm}$. If the 
resolution error is too large, is there an error trend so that this resolution problem can possibly be compensated for? These are some of the questions that can be asked from the ranging accuracy point of view.

\subsection{Thesis Summary}

Chapter 2 provides more info on the VT-DBR laser and its tuning mechanism along with a basic theoretical background on linewidth needed to aid in understanding the experiments performed in Chapter 3 and Chapter 4 .

Chapter 3 outlines an experiment done to capture the laser linewidths across the laser's sweep range and analysis of what those linewidths indicate along with how one may achieve minimum linewidth.

Chapter 4 outlines an experiment done to test the feasibility of ranging by the laser within optical fibers. Analysis of how one can calculate range from the data retrieved and how accurate the ranging is, is discussed.

Chapter 5 shares conclusions with regards to the investigation done and future work that is necessary to further investigate into the feasibility of an FMCW laser on future autonomous systems. 


\section{Chapter 2. The Insight VT-DBR Laser and Linewidth}

In order to discuss aspects of the device under test, the Insight VT-DBR akinetic laser, it would be helpful to understand what the VT-DBR akinetic laser is before describing the specific capabilities of the Insight packaged laser. After discussing the capabilities of the Insight laser, the characteristics of interest of the laser in the application of ranging is discussed to be able to give a basic theoretical basis for the experiments found in the following Chapters, 3 and 4 .

\subsection{Laser Components and the VT-DBR Laser}

Three main components are necessary for a conventional laser to produce an optical output and they include a gain medium, an optical resonator, and a pump. First, the gain medium is a medium which amplifies the power of light in order to compensate for resonator losses. Different types of material with the necessary properties such as a gas, liquid, or solid can be used, but in the VT-DBR laser specifically, a semiconductor is used. Second, the optical resonator is placed around the gain medium to act as a source of a feedback for the system and is composed of two parallel mirrors, one which is fully reflective and another which is partially reflective to allow light to escape the laser cavity. Last, the pump is the source of energy for 
the laser and injects electrical or optical energy into the medium to stimulate the process of light emission [13]. Figure 2-1 illustrates the configuration of a gain medium, optical resonator, and pump of a laser.

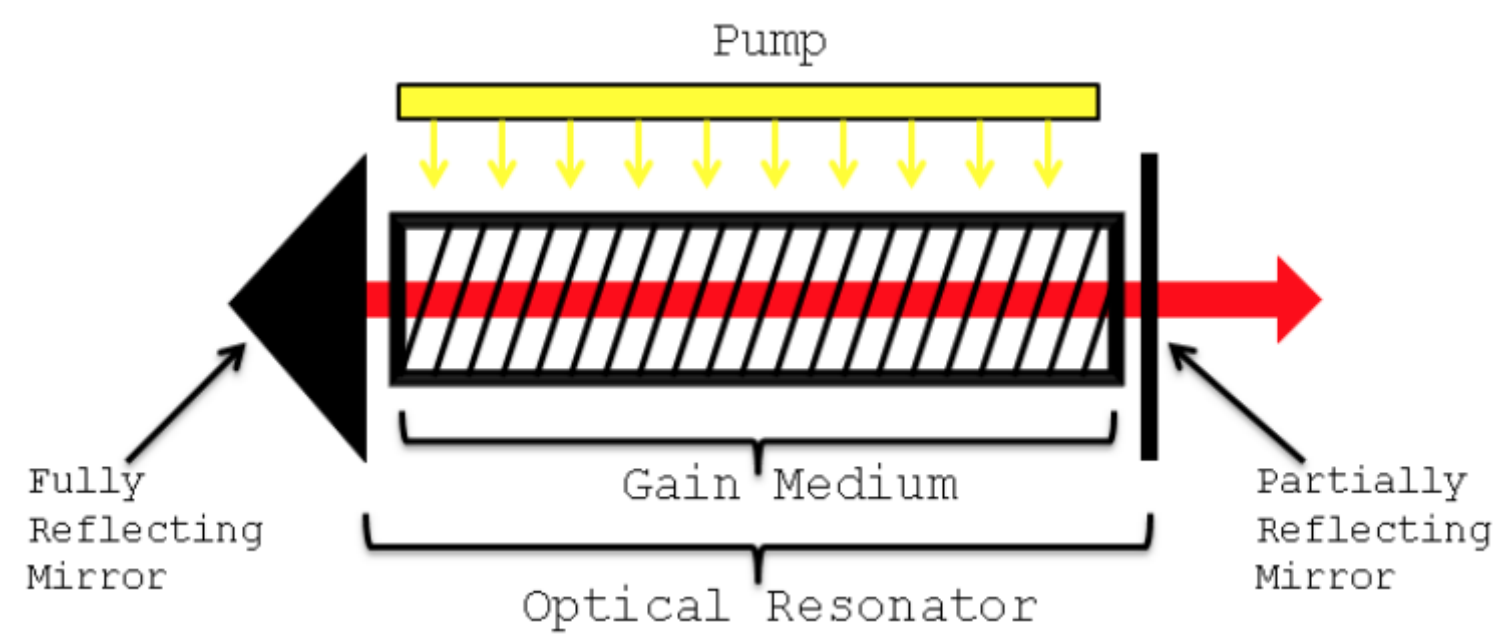

Figure 2-1 - A conventional laser configuration of a gain medium, pump, and optical resonator consisting of a fully reflective mirror and partially reflective mirror [13].

The Vernier-Tuned Distributed Bragg Reflector (VT-DBR) akinetic laser source is a high-speed, all-semiconductor, tunable laser (controllable over a certain wavelength range) [15]. Because this laser wavelength is controllable, it is ideal for a sweep of frequencies, inherent in the FMCW method commonly found today in radar.

Moving on from the conventional laser, and discussing the specific laser used in this study, The VT-DBR laser's foundation works by using two distributed Bragg-reflector (DBR) mirrors on each end of its optical cavity to select the oscillatory wavelength [16]. The mirrors reject the 
transmission of a harmonically related set of wavelengths, depending on the length of separation of the two mirrors, and the rejected light resonates within the laser cavity in the electrically pumped gain medium where a single mode commonly reflected by the mirrors is amplified [15].

The distance between the DBR mirrors of the VT-DBR laser is altered by application of different electric currents into the semiconductor materials the mirrors are composed of, changing their refractive index [17]. Reflective angle of incidence is changed from a change in refractive index of a material, which ultimately changes the distance seen by light between the two mirrors. In turn, this affects the light to be rejected by the mirrors, changing the wavelength output of the laser.

The VT-DBR has 5 control currents to perform wavelength tuning which include the back mirror (bm), gain amplifier, phase, front mirror (fm), and semiconductor optical amplifier currents (soa) which can be seen in Figure 2-2. 


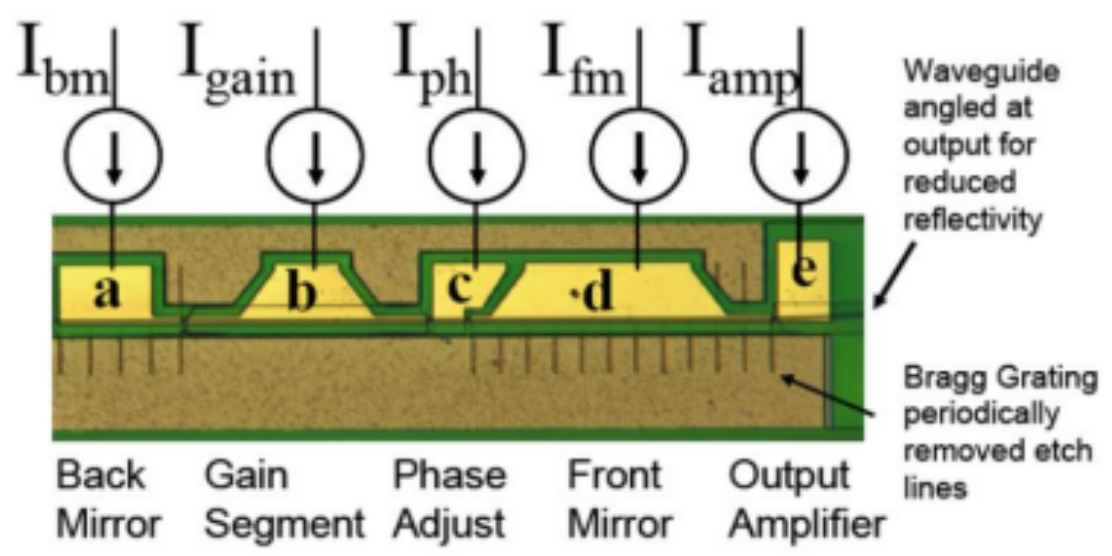

Figure 2-2 - The 5 control currents of the VT-DBR to control wavelength tuning [18]

The front mirror (fm), back mirror (bm), and phase sections which can also be seen in Figure 2-2, are responsible for wavelength selection [19]. These currents can change the refractive index of the DBR mirrors. The semiconductor optical amplifier (soa) and gain sections allow control of the power output of the laser. "Vernier" in VT-DBR refers to the Vernier effect used in tuning the laser from the comb reflection spectra found in the DBR structures [15]. What makes this laser superior to other tunable lasers is the "akinetic" in "VT-DBR akinetic laser source" title which means that there are no moving parts. The advantage of this is that because there are no moving parts the wavelengths can be controlled more quickly. 


\subsection{Capabilities of Insight Laser}

The Insight Akinetic Laser source, which is the laser used throughout the experiments in Chapters 3 and Chapter 4, provides the user interface and packaging of the VT-DBR laser discussed in the previous section. Digital control is utilized to change the control current pumps against time in order to provide an accurate control of optical wavelengths. A default sample clock of $400 \mathrm{MHz}$ is used and trigger signals are generated by the laser to indicate when measurements are valid to be taken. Figure 2-3 gives a visual representation of the front panel of the Insight laser source.

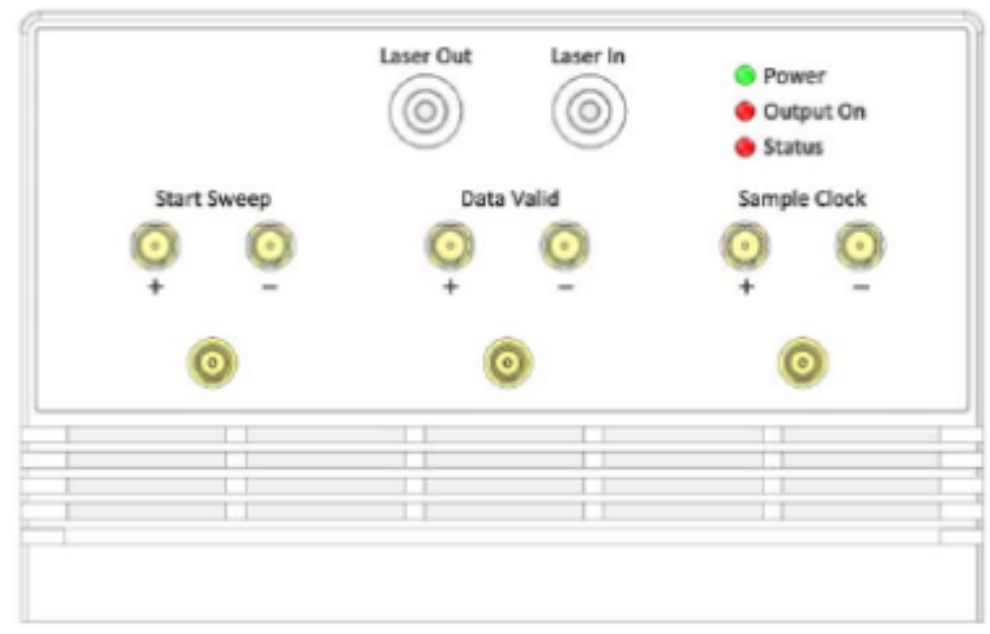

\section{Figure 2-3 - Front Panel of the Insight Akinetic Laser source $[20]$.}

As can be seen in Figure 2-3, "Laser out" is the laser output of the laser while "Laser in" in is the input to a internal acquisition device. "Start Sweep" is a digital signal output that allows one to know the duration of a 
laser sweep and "Data Valid" output is also a digital

signal output that notifies the user when points in a laser sweep are valid or not. The outputs of the laser can be seen in a LABVIEW oscilloscope via use of 5105 PCI

Digitizer ADC and a 5105 Acquisition program as seen in Figure 2-4

Waveform Graph 2

"Start Sweep"

Waveform Graph 3

"Data Valid"

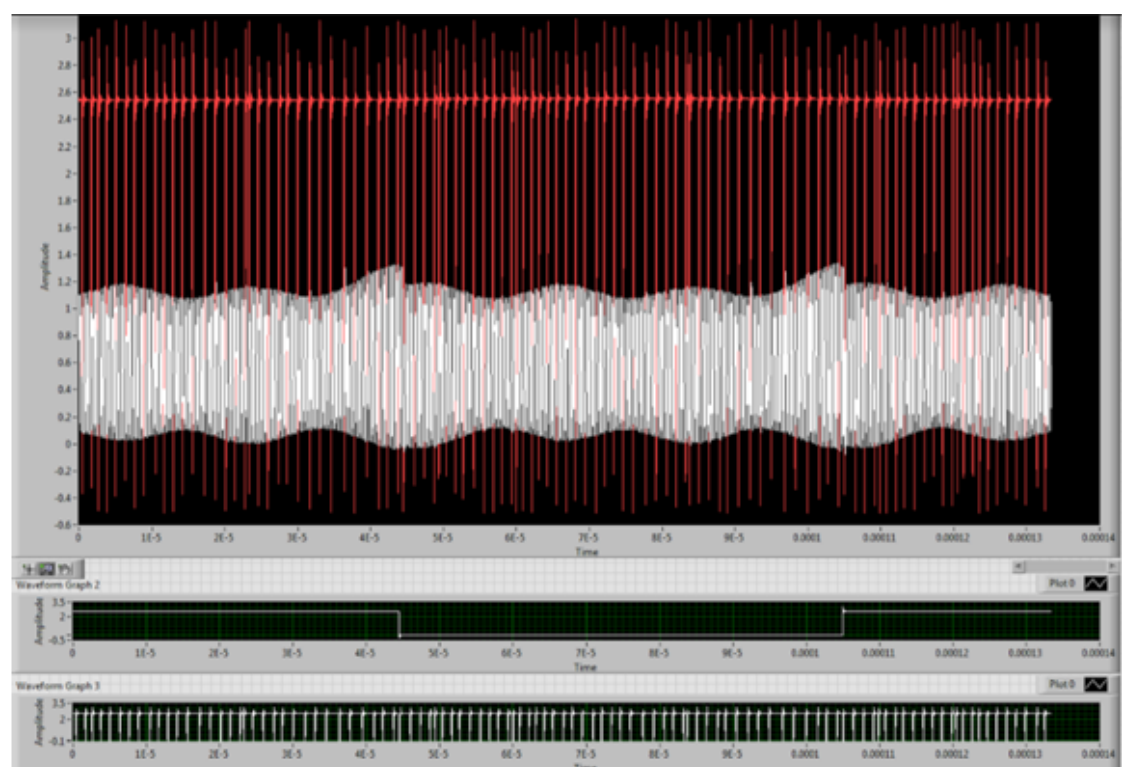

Figure 2-4 - 5105 Acquisition program with time domain waveform data and waveforms of laser outputs including "Data Valid" and "Start Sweep".

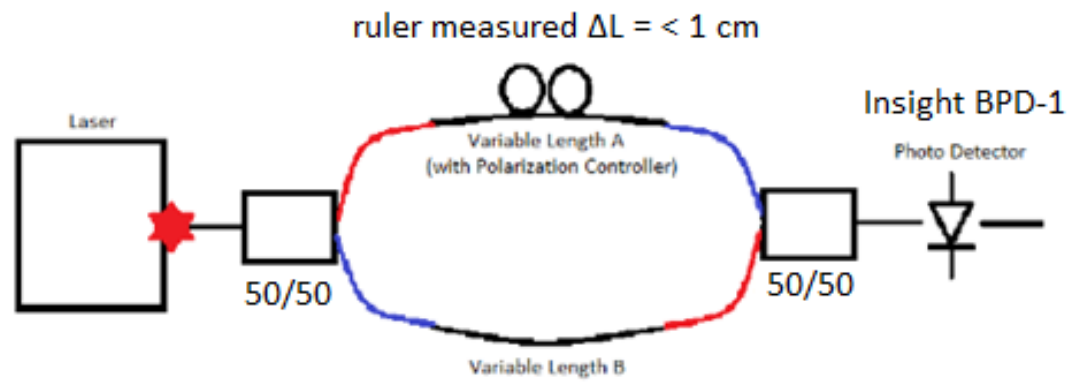

5105 Acquisition

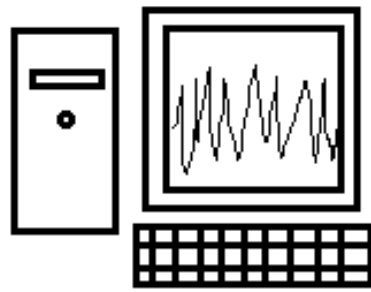

Figure 2-5 - Interference fringe test setup for a ruler measured path length difference of $1 \mathrm{~cm}$. 
The white waveform seen in Figure 2-4 is an example of a time domain waveform that is under analysis which is the output of the interference fringe test setup shown in Figure 2-5. The red waveform overlapped with the white waveform is the digital data valid signal that is high to indicate valid laser output and low to indicate invalid laser output. Waveform Graph 2 shows the waveform of the "Start Sweep" output of the Insight laser and as can be seen is indicating the beginning and end of sweeps at its rising and falling edges. Waveform Graph 3 is the data valid output of the laser in a separate graph from the time domain waveform under analysis.

The specific Insight laser model used throughout this thesis is Model SLE-101 and has a sweep range of 1522.13 to $1566.18 \mathrm{~nm}$.

\subsection{Linewidth and Coherence}

The linewidth $\Delta v$ of a laser is the width of its optical spectrum $3 \mathrm{~dB}$ down from the peak or at half power. A laser spectrum usually has Lorentzian-shaped central peak, small sidebands located closer to the central peak caused by relaxation oscillations, and small sidemodes (cavity frequencies) located farther from the central peak [21]. A typical optical spectrum is shown in Figure 2-6 below. 


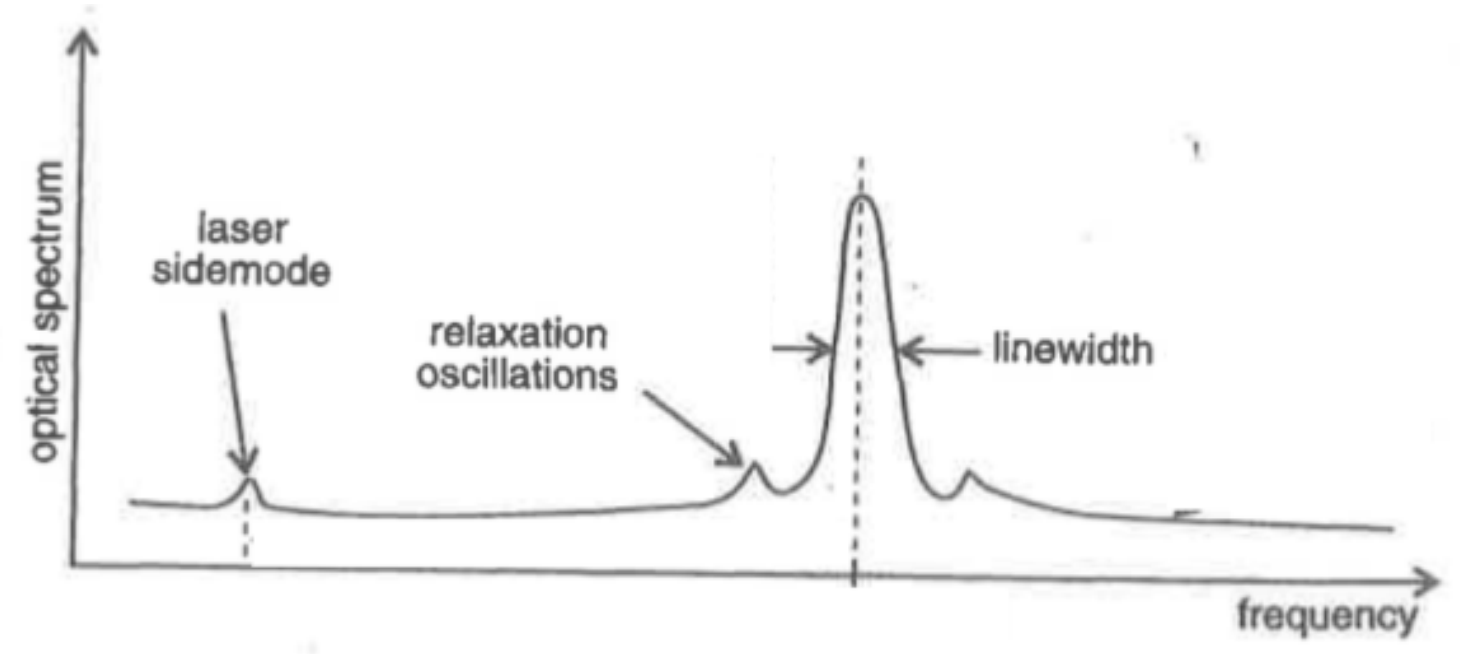

Figure 2-6 - Typical Lorentzian-shaped central peak laser spectrum with relaxation oscillations and sidemodes [21].

In the case of linewidth for our specific application, the narrower the better. For example a range/sensitivity of $100 \mathrm{~m}$ as was outlined in Table 1 of Section 1.3 in Chapter 1 within optical fiber requires a narrow linewidth of 649.6 $\mathrm{kHz}$. Larger range/sensitivity, translates to a shorter required linewidth. This is due to the fact that linewidth is related to temporal coherence, which is characterized by coherence time or coherence length. Coherence time is a measure of spectral purity of a laser wavelength or frequency over time and a narrower linewidth is related to a longer coherence time and coherence length. The mathematical relationship between linewidth and coherence time $\tau_{c}$ is shown as follows:

$$
\tau_{c}=\frac{1}{\pi \Delta v}
$$


The coherence length is the coherence time multiplied by the velocity of light within optical fiber which is:

$$
v_{g}=\frac{c}{n_{g}}
$$

Where the group velocity index $n_{g}$ for optical fiber is 1.47 , leading to the coherence length equation of:

$$
L_{c}=\tau_{c} v_{g}
$$

Coherence time/length can be reduced by random events like spontaneous emission in the laser cavity, which can alter phase or frequency of the laser output. This concept is illustrated in Figure 2-7.

(a)

(b)

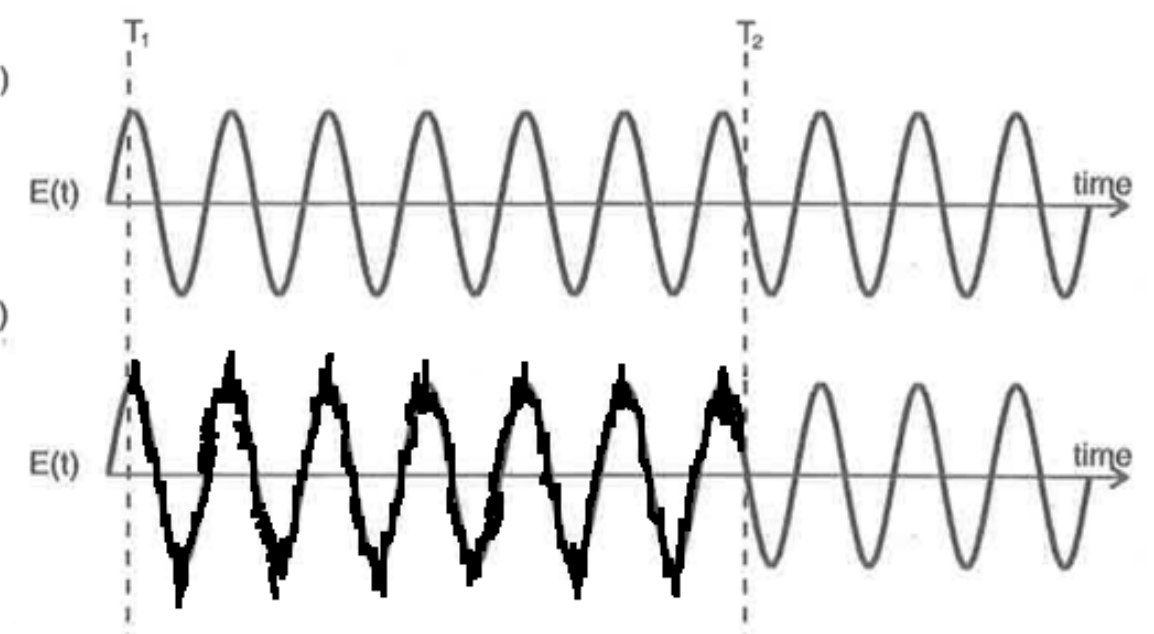

Figure 2-7 - Concept of coherence time with an example of (a) coherent light, and (b) short coherence [21]. As can be observed in Figure 2-7, the coherence is longer in (a) and shorter in (b). Looking between the time intervals of $T_{1}$ and $T_{2}$, the coherence is longer in (a) because phase is predictable across the interval, while in 
(b) there are random phase or frequency jumps which causes the phase to be unpredictable across the interval.

In two path interferometers, the degree to which an optical wave interferes with a delayed version of itself depends on the coherence time of the wave with respect to the optical delay [21]. As such, coherence length and coherence time plays a large role in the ranging with interference fringes experiment done in Chapter 4 and due to that large role played, linewidth is the subject of investigation in Chapter 3 . 


\section{Chapter 3. Laser Linewidth Characterization}

With the importance of linewidth emphasized in Section 2.4 of Chapter 2, and the goals outlined in Table 1 in Section 1.3 of Chapter 1 , an experiment was done to be able to efficiently characterize the Insight Laser's linewidth. For FMCW, the laser must be swept at range of frequencies. As such, it made sense to step the laser across a range of frequencies that could be possible candidates for a sweep range to be selected that would utilize wavelengths with minimal linewidth compared to say wavelengths in another range with larger linewidths.

\subsection{Laser Linewidth Test Setup and Theory}

With the Insight Laser Control Program the laser can be set in fixed wavelength mode. The interface is shown in Figure 3-1. 


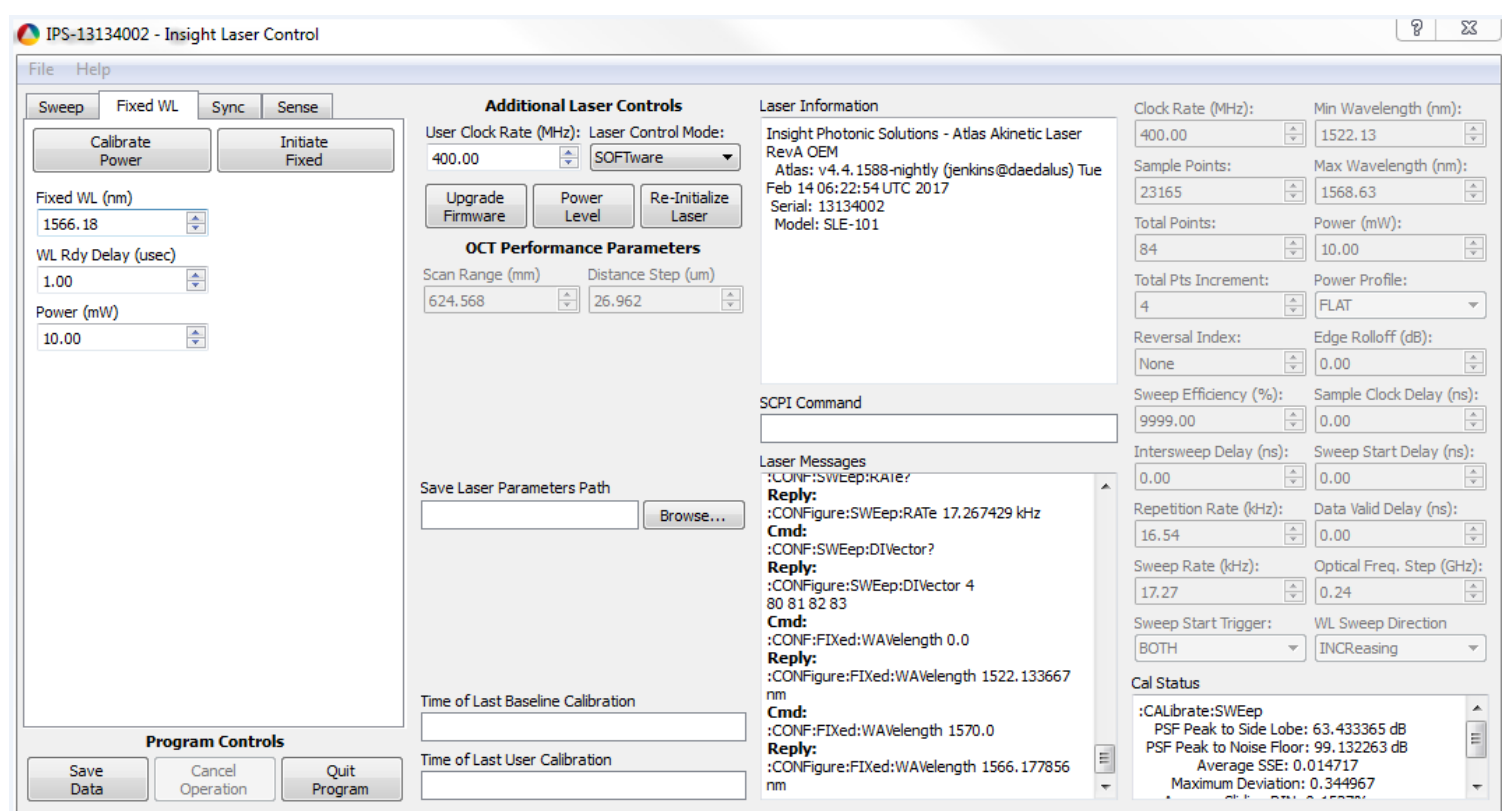

Figure 3-1 - Insight Laser Control Program fixed wavelength output interface [20].

The fixed wavelength interface allows the user to output the laser at a fixed wavelength with modifiable power. The output of the laser in this mode can range from wavelengths of $1522.13 \mathrm{~nm}$ to $1566.18 \mathrm{~nm}$. The output of the laser at a wavelength of $1540 \mathrm{~nm}$ and at a power of $5 \mathrm{dBm}$ is shown in a Optical Spectrum Analyzer (Agilent 86140B) to visually highlight the characteristics of the laser in the frequency spectrum in Figure 3-2. 


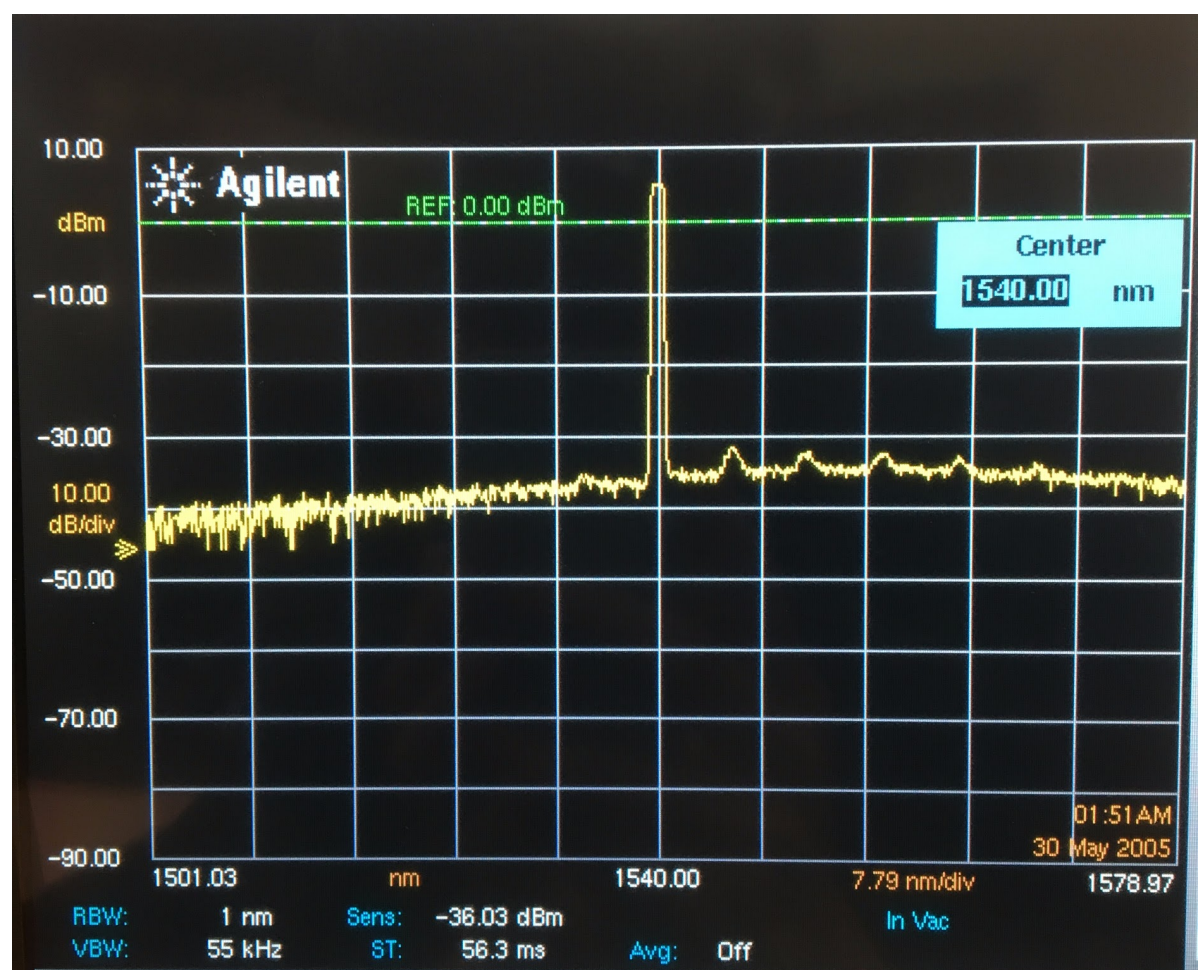

Figure 3-2 - Fixed $1540 \mathrm{~nm}$ output of laser shown in optical spectrum analyzer.

Because there is a large number of different wavelengths that can be output, in order to get a characteristic of the entire range of the laser in the most efficient way possible, it was determined that $1 \mathrm{~nm}$ step would be sufficient to get an understanding of linewidth variability across the range. More specifically, the laser linewidth would be observed across the range of 1523 to $1566 \mathrm{~nm}$ in $1 \mathrm{~nm}$ increments.

With the laser under test settings determined and basic methodology for the how the laser is to be tested figured out, the next step was to determine the method of measuring the linewidth of the laser and the delayed self- 
homodyne method was chosen. The delayed self-homodyne method is a simple way to measure the linewidth of a laser and offers a very high resolution compared to other methods of measuring linewidth due to the simple usage of an optical interferometer with low-loss fiber optic delays [21]. In this method, there is a photodetector that must receive a combined optical field, consisting of one field which is delayed and another that is minimally delayed, which is why an interferometer is necessary. There are many different types of interferometers that can be used for the purpose of delayed self-homodyne including Mach-Zehnder, Micheson, and Fabry-Perot interferometers [21]. Visual representations of the different types of interferometers can be seen in Figure 3-3 [21], however a Mach-Zehnder interferometer was chosen to move forward with due to simplicity and availability. 


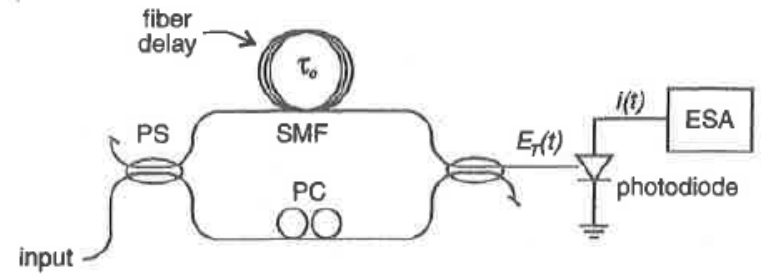

Mach-Zehnder interferometer
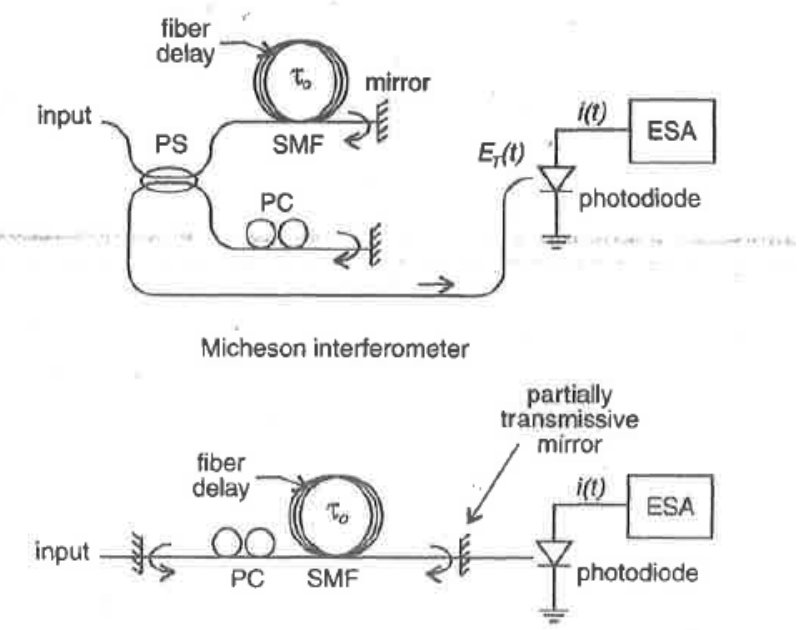

Low-finess Fabry-Perot interferometer

Figure 3-3 - Mach-Zehnder, Micheson, and Fabry interferometers seen in delayed self-homodyne measurement setups [21].

The translation from optical to electrical spectrum can be seen in Figure 3-4 [21]. The spectrum shape of semiconductor lasers can be approximated by a Lorentzianshaped profile and for such shapes such as Lorentzian and Gaussian, lineshapes are preserved from the optical to the electrical through the autocorrelation operation [21] • 


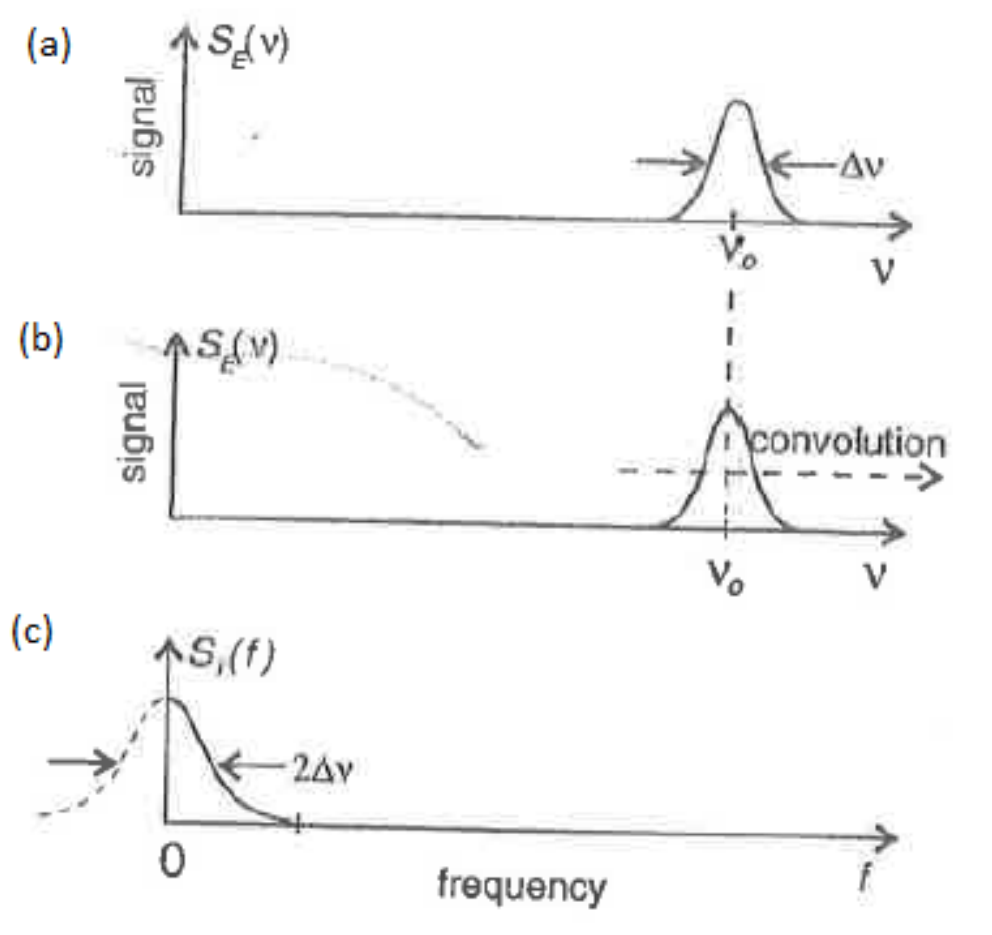

Figure 3-4 - (a) Laser lineshape mixing with (b) delayed self for (c) output electrical spectrum shape [21].

As can be seen in Figure 3-4, the linewidth appears in the electrical spectrum with half of the full spectral shape shown (due to the mixed spectrum centered about $0 \mathrm{~Hz}$ ) of which the width of the half spectrum at $3 \mathrm{~dB}$ down is the linewidth.

\subsection{Laser Linewidth Test}

With a basic theoretical background and a method of capturing linewidth determined, the experimental setup to capture the linewidths of fixed wavelengths across the wavelength range able to be output by the Insight laser was begun. As hinted, the experimental setup for the delayed self-homodyne is simple. The Insight laser was connected to 
an off the shelf $3.5 \mu \mathrm{s}$ delay Mach-Zehnder Interferometer with a knob for polarization (Hewlett Packard 11980A) for the summation of the laser output with itself. This was then connected to the input of a photodetector to convert from the optical to the electrical spectrum (optical power into electrical current). The output of the photodetector was then input into an off the shelf electrical spectrum analyzer (Agilent Technologies N9000A) for display of the electrical spectrum to the user. A visual representation of the experimental setup for linewidth characterization of the Insight laser can be seen in Figure 3-5.

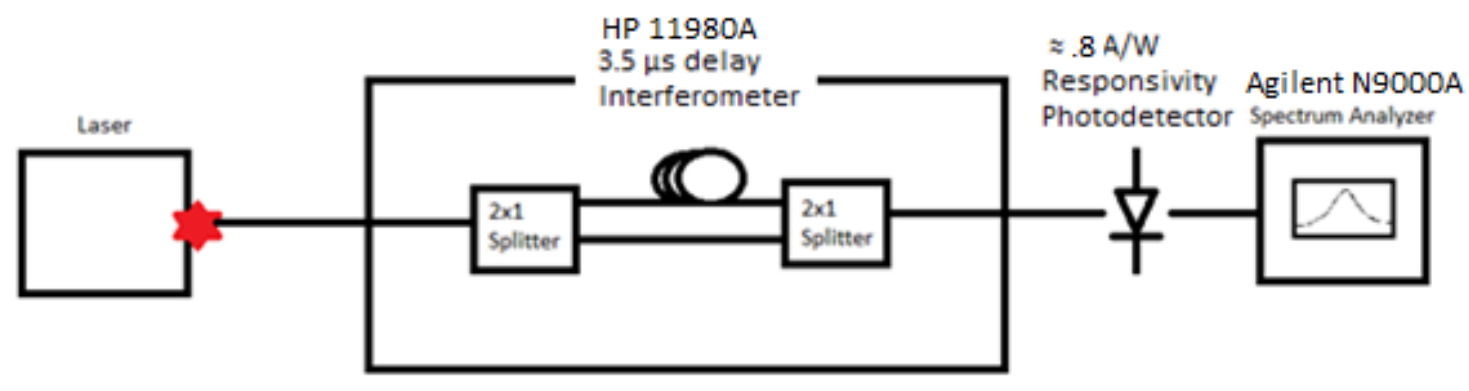

Figure 3-5 - Insight laser delayed self-homodyne experimental test setup: laser is output into interferometer for mixing with delayed self into photodetector to observe output spectrum in electrical spectrum analyzer.

With the experimental setup ready, the output wavelength range of the laser was run through. As mentioned before, steps of $1 \mathrm{~nm}$ of fixed wavelength were done across the range of 1523 to $1566 \mathrm{~nm}$. It must be noted that the Insight laser fixed wavelength interface was not able to 
output at exactly 1556 and $1561 \mathrm{~nm}$ and in order to keep consistency of data, they were skipped.

As mentioned before on why linewidth is important for laser ranging in Section 2.4 in Chapter 2, linewidth has a relation to coherence time and as a result coherence lengths. As such, after the laser linewidth spectrum was captured for the range of the laser, linewidth was extracted. With the linewidth $\Delta v$, coherence time $\tau_{c}$ and coherence length $L_{c}$ was calculated as follows:

$$
\begin{aligned}
& \text { group velocity index } n_{g}=1.47 \text {, for optical fiber } \\
& \text { velocity of light within fiber } v_{g}=\frac{c}{n_{g}} \\
& \text { coherence time } \tau_{c}=\frac{1}{\pi \Delta v} \\
& \text { coherence length } L_{c}=\tau_{c} v_{g}
\end{aligned}
$$

Further, in order to gain an understanding of how the laser currents play into the linewidth, the laser currents at play during each of the fixed wavelength outputs were gathered from factory data provided by Insight Photonics [22]. Please refer to Appendix A for the results of this experiment in tabular format for the linewidths captured from $1523 \mathrm{~nm}$ to $1566 \mathrm{~nm}$ and the corresponding coherence times, coherence lengths, and laser currents. Figure 3-6 highlights the format in which the data was taken for example purposes for a fixed wavelength output of $1523 \mathrm{~nm}$. 


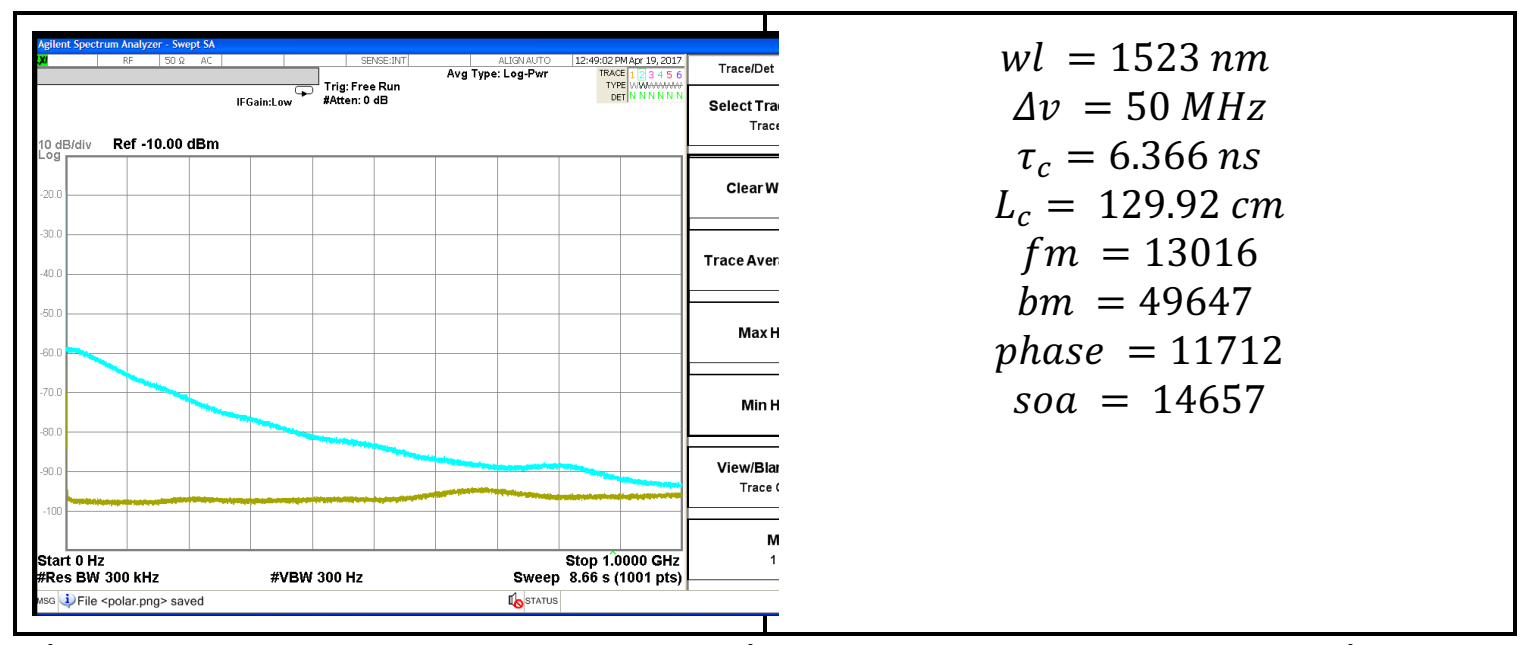

Figure 3-6 - Example of experimental data taken for fixed wavelength output of $1523 \mathrm{~nm}$.

Although the linewidth $\Delta v$ provides the width of the lineshape at $3 \mathrm{~dB}$ down from the peak, it is not enough to highlight the rest of the lineshape that is shown. For example, the $3 \mathrm{~dB}$ point will only show how close the lineshape is hugging the $0 \mathrm{~Hz}$ peak but does not highlight how much the lineshape spreads out the further from $0 \mathrm{~Hz}$ one may get. In order to capture this data, the width from $0 \mathrm{~Hz}$ was captured in terms of $\mathrm{dB}$ points. So instead of capturing just the linewidth ( $3 \mathrm{~dB}$ down), the width from 0 $\mathrm{Hz}$ was captured at $10 \mathrm{~dB}$ down, $20 \mathrm{~dB}$ down, and $30 \mathrm{~dB}$ down and was compared with the theoretical values of a Lorentzian spectral shape, which were calculated utilizing Figure 3-7. 


\begin{tabular}{cc}
\hline Measured Point & Displayed Width \\
\hline$-3 \mathrm{~dB}$ & $\Delta \nu$ \\
$-10 \mathrm{~dB}$ & $\sqrt{9} \Delta \nu$ \\
$-20 \mathrm{~dB}$ & $\sqrt{99} \Delta \nu$ \\
$-30 \mathrm{~dB}$ & $\sqrt{999} \Delta v$ \\
\hline
\end{tabular}

Figure 3-7 - Theoretical Lorentzian spectral shape at measured points of $-3 \mathrm{~dB},-10 \mathrm{~dB},-20 \mathrm{~dB}$, and $-30 \mathrm{~dB}$ [21].

The frequencies at these points were captured and this experimental data for the fixed wavelengths of 1523 to 1566 $\mathrm{nm}$ along with the theoretical Lorentzian values calculated in tabulated form can be found in Appendix B. Figure 3-8 highlights the format in which the data was recorded for example purposes for a fixed wavelength output of $1523 \mathrm{~nm}$.

\begin{tabular}{|c|c|c|}
\hline $\begin{array}{l}\text { Laser } \\
\text { Wavelength }\end{array}$ & $\begin{array}{l}\text { Experimental Laser } \\
\text { Lineshape }\end{array}$ & $\begin{array}{l}\text { Lorentzian Laser } \\
\text { Lineshape }\end{array}$ \\
\hline $1523 \mathrm{~nm}$ & $\begin{array}{c}\Delta v=50 \mathrm{MHz} \\
\Delta v_{10 \mathrm{~dB}}=160 \mathrm{MHz} \\
\Delta v_{20 \mathrm{~dB}}=350 \mathrm{MHz} \\
\Delta v_{30 \mathrm{~dB}}=700 \mathrm{MHz}\end{array}$ & $\begin{array}{c}\Delta v=50 \mathrm{MHz} \\
\Delta v_{10 \mathrm{~dB}}=150 \mathrm{MHz} \\
\Delta v_{20 d B}=497.5 \mathrm{MHz} \\
\Delta v_{30 d B}=1580 \mathrm{MHz}\end{array}$ \\
\hline
\end{tabular}

Figure 3-8 - Example of experimental data recorded of width at various $d B$ down from peak for fixed wavelength output of $1523 \mathrm{~nm}$. 


\subsection{Laser Linewidth Test Results and Analysis}

As can be initially observed from the laser linewidth test tabulated in Appendix A, it can be seen that there is

a large variation between the different wavelengths in terms of linewidth with the largest linewidth captured to be $300 \mathrm{MHz}$ at a fixed wavelength laser output of $1562 \mathrm{~nm}$ leading to a calculated minimum coherence time of $1.061 \mathrm{~ns}$ and coherence length of $21.65 \mathrm{~cm}$. The smallest linewidth captured to be at $50 \mathrm{MHz}$ found at fixed wavelength laser outputs of $1523,1525,1539,1545$, and $1546 \mathrm{~nm}$ leading to a maximum coherence time of $6.366 \mathrm{~ns}$ and coherence length of $129.92 \mathrm{~cm}$.

Thus, the largest linewidth captured by this experiment is $600 \%$ that of the smallest linewidth captured. In order to gain a clearer representation of how linewidth varied with wavelength output by the laser, they were graphed against each other and can be seen in Figure 3-9. 


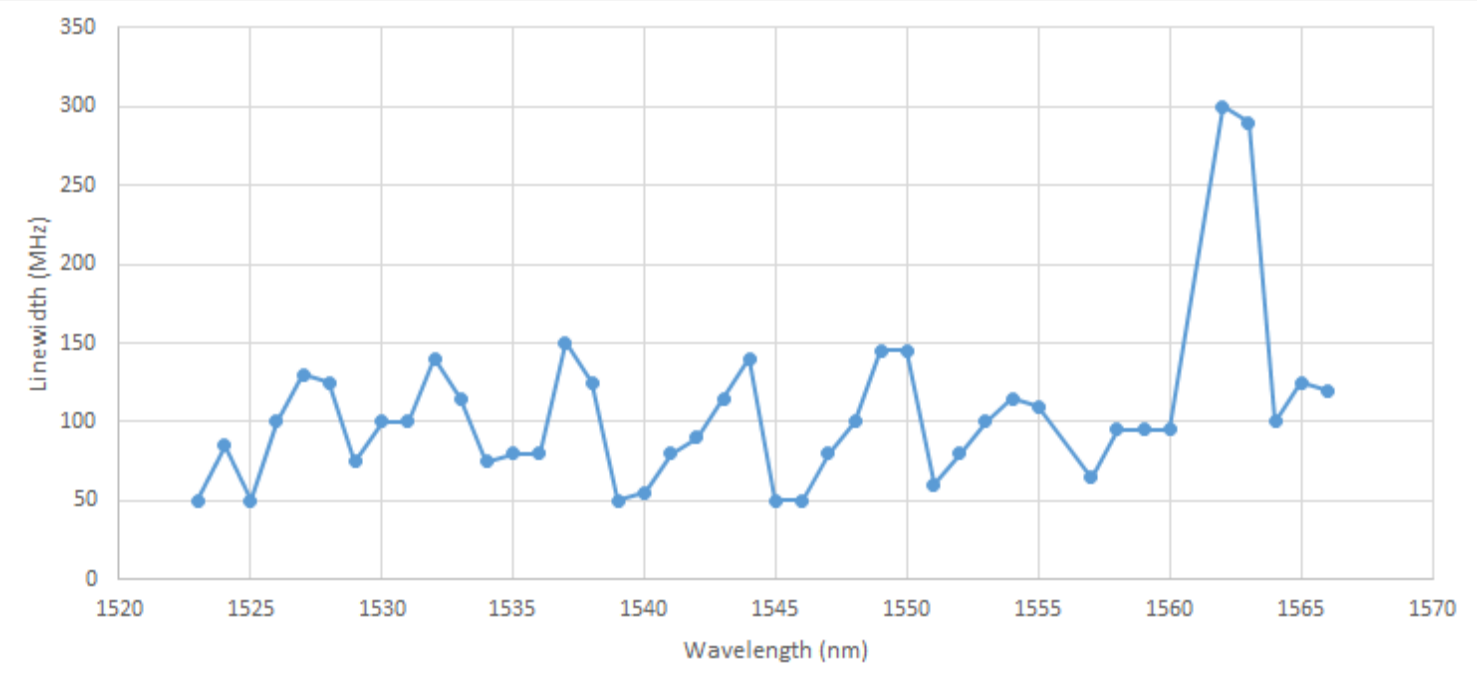

Figure 3-9 - Laser linewidth graphed against fixed wavelength laser output.

As can be observed in Figure 3-9, across the range there is a large up and down variability of the linewidth from 1523 to $1566 \mathrm{~nm}$. What is immediately obvious from the graph however, is how far out the linewidth is at 1562 and $1563 \mathrm{~nm}$ compared to the linewidth at the rest of the wavelengths. Besides 1562 and $1563 \mathrm{~nm}$ with linewidths of 300 and $290 \mathrm{MHz}$, the next largest linewidth is found to be $150 \mathrm{MHz}$ at $1537 \mathrm{~nm}$ which is approximately half of the linewidth of the two maximum. The wavelengths and corresponding linewidths at which there is an obvious peak and the wavelengths at which there is a obvious trough compared to data points in the vicinity in the up and down linewidth nature of Figure 3-9 is captured in Table 3 and Table 4 respectively. Please note 1549 and $1550 \mathrm{~nm}$ were both 
included in Table 3 due to their equivalence in linewidth. For the same reason 1545 and 1546 were both included in Table 4 .

Table 3 - Peaks in linewidth found at various wavelength outputs of laser.

\begin{tabular}{|l|l|}
\hline \multicolumn{1}{|c|}{$\lambda$} & \\
\hline $1524 \mathrm{~nm}$ & $85 \mathrm{MHz}$ \\
\hline $1527 \mathrm{~nm}$ & $130 \mathrm{MHz}$ \\
\hline $1532 \mathrm{~nm}$ & $140 \mathrm{MHz}$ \\
\hline $1537 \mathrm{~nm}$ & $150 \mathrm{MHz}$ \\
\hline $1549 \mathrm{~nm}$ & $140 \mathrm{MHz}$ \\
\hline $1550 \mathrm{~nm}$ & $145 \mathrm{MHz}$ \\
\hline $1554 \mathrm{~nm}$ & $145 \mathrm{MHz}$ \\
\hline $1562 \mathrm{~nm}$ & $300 \mathrm{MHz}$ \\
\hline
\end{tabular}

Table 4 - Troughs in linewidth found at various wavelength outputs of laser.

\begin{tabular}{|l|l|}
\hline \multicolumn{1}{|c|}{$\lambda$} & \\
\hline $1523 \mathrm{~nm}$ & $50 \mathrm{MHz}$ \\
\hline $1525 \mathrm{~nm}$ & $50 \mathrm{MHz}$ \\
\hline $1529 \mathrm{~nm}$ & $75 \mathrm{MHz}$ \\
\hline $1534 \mathrm{~nm}$ & $75 \mathrm{MHz}$ \\
\hline $1539 \mathrm{~nm}$ & $50 \mathrm{MHz}$ \\
\hline $1545 \mathrm{~nm}$ & $50 \mathrm{MHz}$ \\
\hline $1546 \mathrm{~nm}$ & $50 \mathrm{MHz}$ \\
\hline $1551 \mathrm{~nm}$ & $60 \mathrm{MHz}$ \\
\hline $1557 \mathrm{~nm}$ & $65 \mathrm{MHz}$ \\
\hline $1564 \mathrm{~nm}$ & $100 \mathrm{MHz}$ \\
\hline
\end{tabular}


Observing the peaks in linewidth as shown in Table 3 , the initial trend that is somewhat apparent is how much the magnitude of the peaks increases over the wavelength range. For example, the first peak is seen at $1524 \mathrm{~nm}$ with a linewidth of $85 \mathrm{MHz}$, while the last peak is seen at $1562 \mathrm{~nm}$ with a linewidth of $300 \mathrm{MHz}$ which is over 3 times larger than the first peak. The general trend from the first peak to the last peak can be found in Figure 3-10.

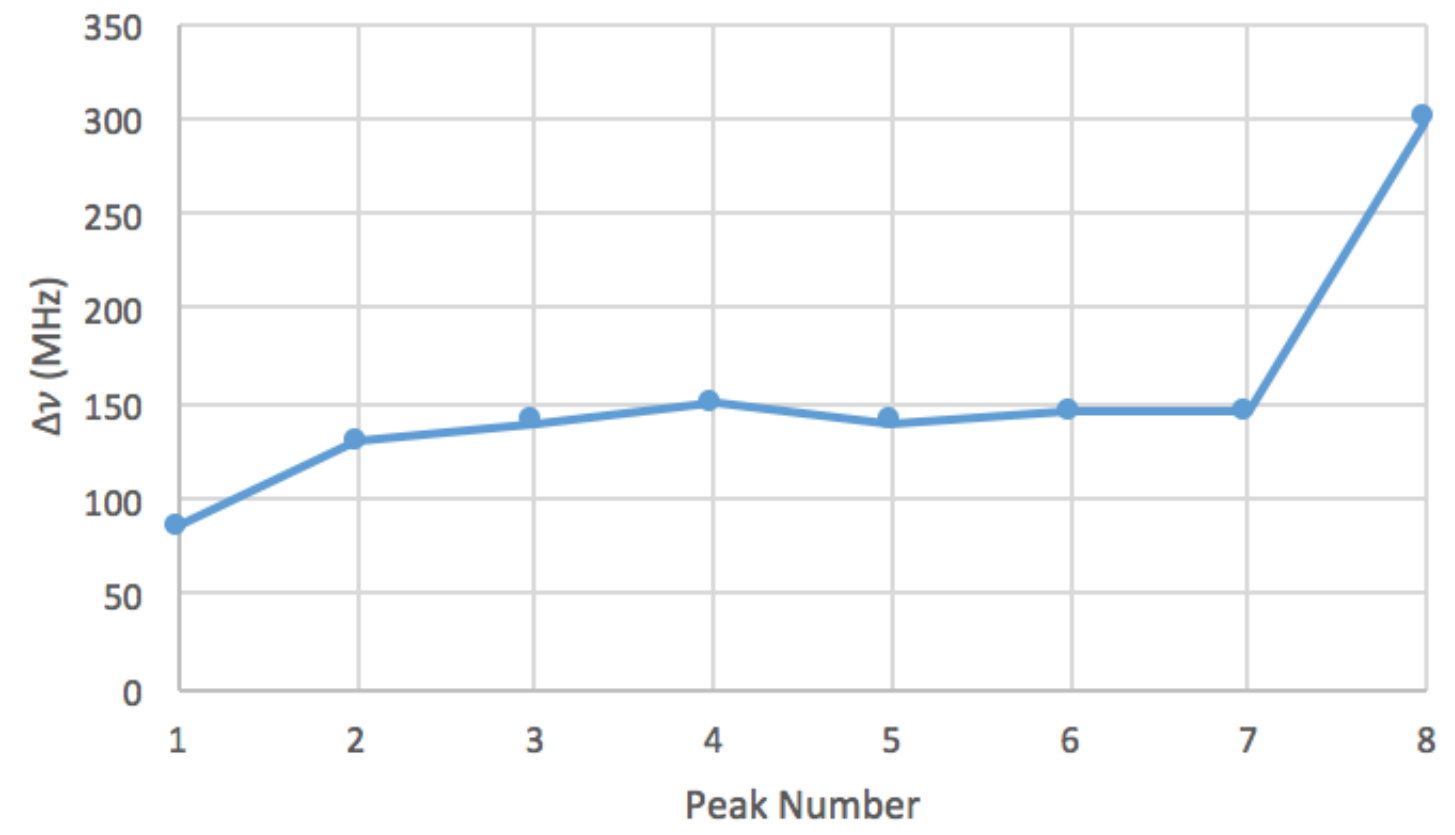

Figure 3-10 - Peak linewidths across the 1523 to $1566 \mathrm{~nm}$ wavelength range under test.

Observing the troughs in linewidth as shown in Table 4, there isn't a very obvious trend besides the fact that the last trough has a higher linewidth than the troughs before it, though not nearly as dramatically as is seen in the linewidth peaks. Otherwise, the rest of the troughs 
seem to hover around $60 \mathrm{MHz}$. The general trend from the first trough to the last trough can be found in Figure 311 .

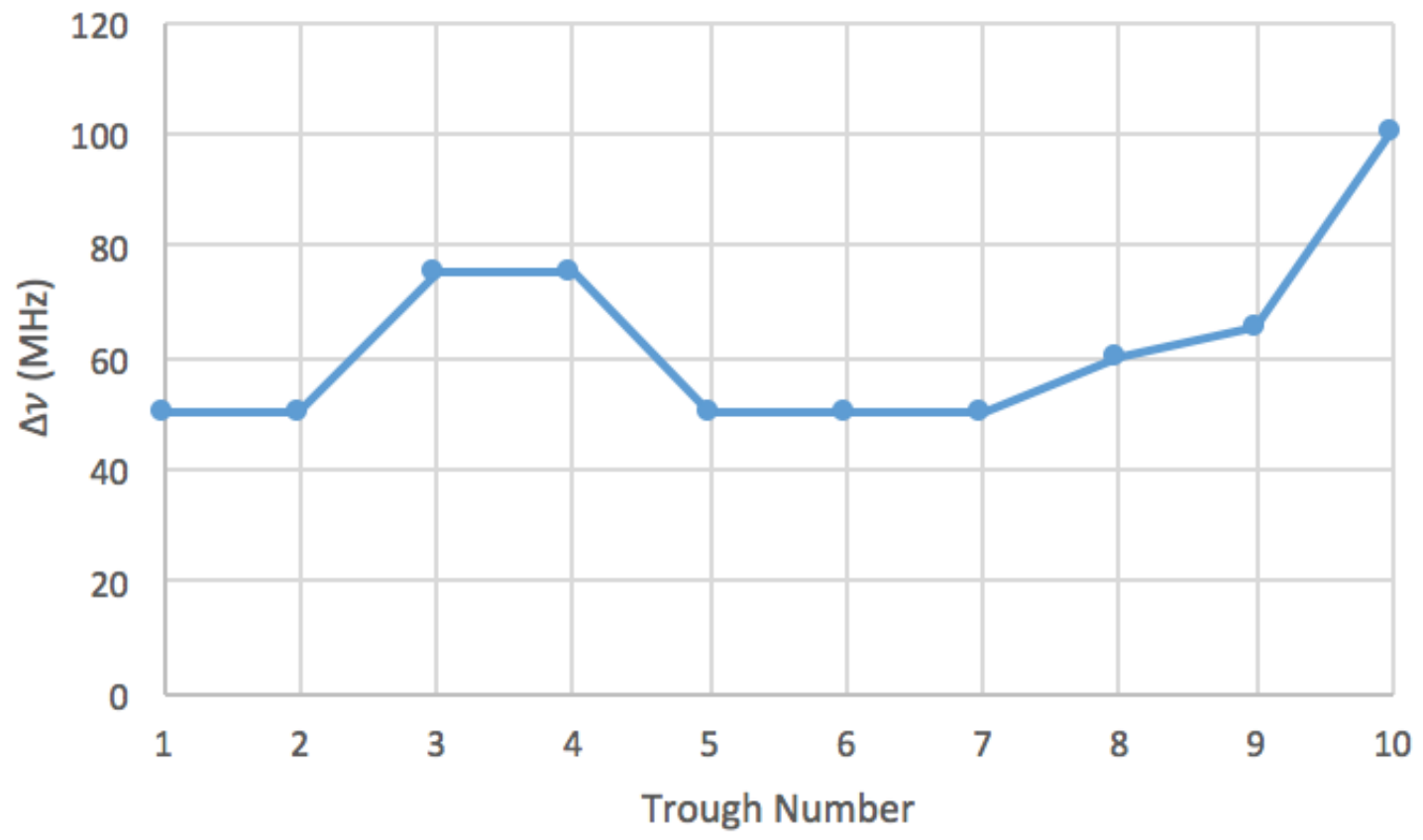

Figure 3-11 - Trough linewidths across the 1523 to $1566 \mathrm{~nm}$ wavelength range under test.

Variation in linewidth is evident with the changes in wavelength, and to get a further understanding of what might cause these changes in linewidth, it was mentioned that the different magnitudes of laser current that are active for a set wavelength may play a factor. To get a visual representation of how these laser currents come into play, the four currents were graphed against wavelength, shown in Figure 3-12. 
(a)

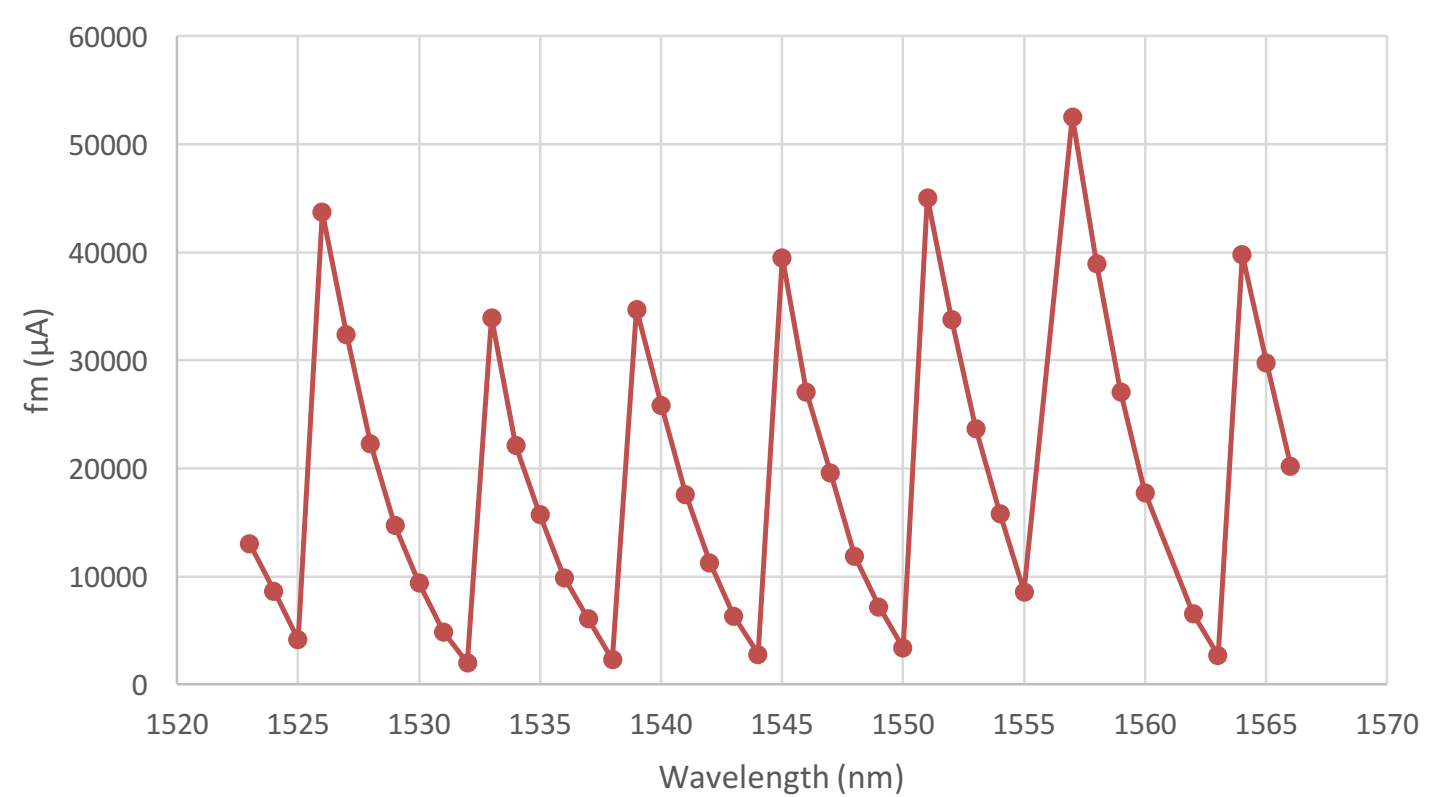

(b)

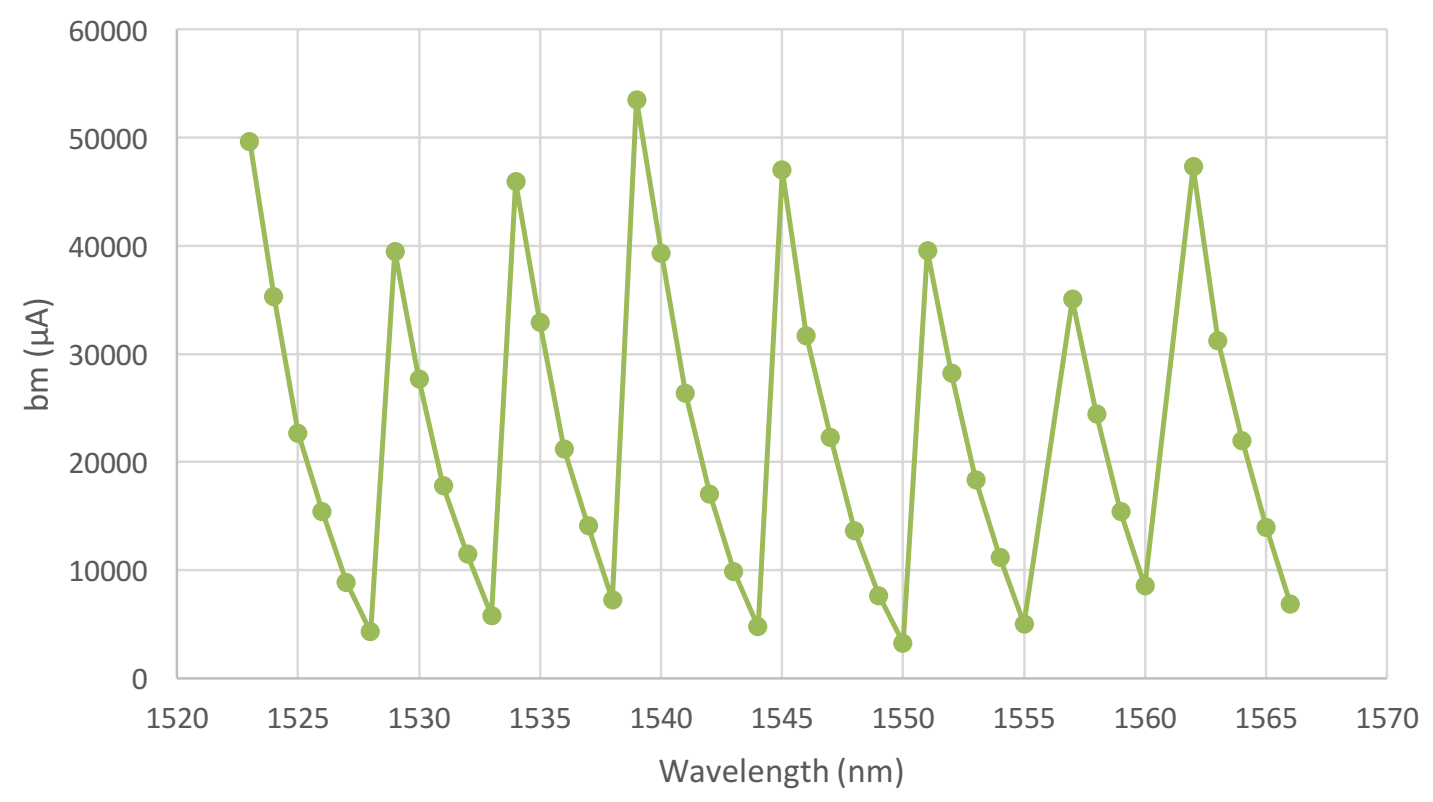


(c)

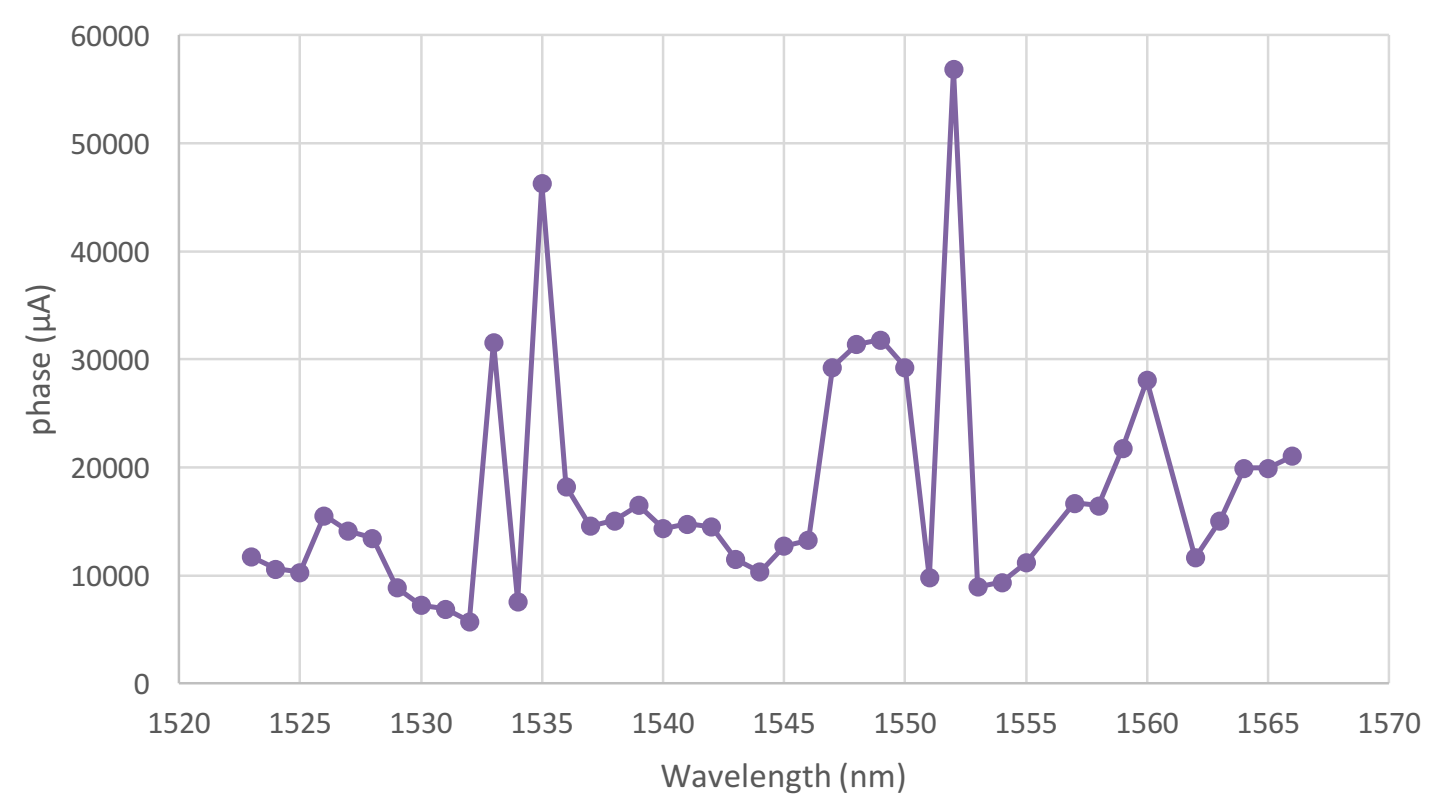

(d)

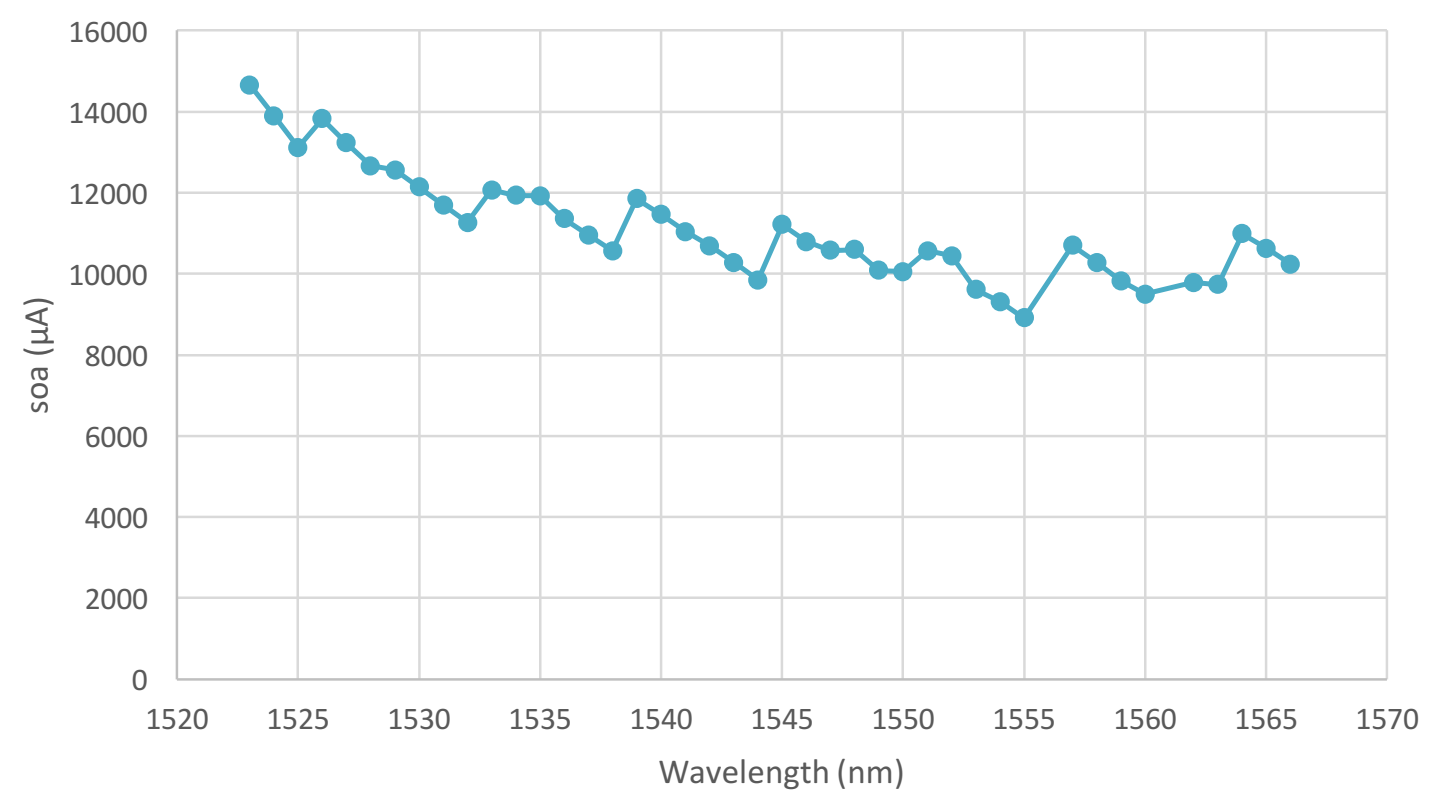

Figure 3-12 - (a) Front mirror (fm), (b) back mirror (bm), (c) phase, and (d) semiconductor optical amplifier (soa) currents variability across the wavelength output range.

As can be observed in Figure $3-12$, the first attributes that are apparent are the large up and down variability of 
both the front mirror and back mirror currents of the laser. The maximum current for the front mirror case being $52534 \mu \mathrm{A}$ at a fixed wavelength of $1557 \mathrm{~nm}$ and the minimum current for the front mirror case being $2022 \mu \mathrm{A}$ at a fixed wavelength of $1532 \mathrm{~nm}$. For the back mirror case the maximum current in this test is $53504 \mu \mathrm{A}$ at a fixed wavelength of $1539 \mathrm{~nm}$, and the minimum current found is $3213 \mu \mathrm{A}$ at a fixed wavelength of $1550 \mathrm{~nm}$. There is also large variability seen with the phase current; however, there does not seem to be an obvious pattern in how the the phase current changes over the range. This is due to the limited amount of data being taken at one $\mathrm{nm}$ increments; an increment which is too large for the phase currents. According to the factory data provided by Insight, the phase current in reality also illustrates an up and down pattern across the wavelength range [22]. In the case of the soa current, its general up and down shape across the wavelength range is similar to the fm and bm currents, but the magnitude of its variability is minimal compared to that of the other currents. Initial observation of the current graphs seem to indicate that the front mirror and back mirror currents with their variability and pattern might be the bigger influences on linewidth. 
With a visual representation of all of the laser currents and linewidth against wavelength output by the laser, the wavelengths at which there were peak and trough linewidths can be observed to get a better indication of the magnitude of the different laser currents at those points. Using the graphs in Figure 3-12 as reference, Table 3 and Table 4 can be expanded with the currents at these wavelengths and is shown in Table 5 and Table 6 .

Table 5 - Peaks in linewidth found at various wavelength outputs of laser with their corresponding laser currents.

\begin{tabular}{|c|l|l|l|l|l|}
\hline$\lambda$ & \multicolumn{1}{|c|}{$\Delta v$} & $\operatorname{fm}(\mu A)$ & $\operatorname{bm}(\mu A)$ & $\operatorname{phase}(\mu A)$ & $\operatorname{soa}(\mu A)$ \\
\hline $1524 \mathrm{~nm}$ & $85 \mathrm{MHz}$ & 8689 & 35317 & 10602 & 13899 \\
\hline $1527 \mathrm{~nm}$ & $130 \mathrm{MHz}$ & 32425 & 8872 & 14129 & 13233 \\
\hline $1532 \mathrm{~nm}$ & $140 \mathrm{MHz}$ & 2022 & 11437 & 5732 & 11264 \\
\hline $1537 \mathrm{~nm}$ & $150 \mathrm{MHz}$ & 6108 & 14066 & 14583 & 10958 \\
\hline $1549 \mathrm{~nm}$ & $140 \mathrm{MHz}$ & 7220 & 7629 & 31801 & 10086 \\
\hline $1550 \mathrm{~nm}$ & $145 \mathrm{MHz}$ & 3377 & 3213 & 29276 & 10050 \\
\hline $1554 \mathrm{~nm}$ & $145 \mathrm{MHz}$ & 15857 & 11153 & 9362 & 9316 \\
\hline $1562 \mathrm{~nm}$ & $300 \mathrm{MHz}$ & 6572 & 47277 & 11626 & 9787 \\
\hline
\end{tabular}

Table 6 - Troughs in linewidth found at various wavelength outputs of laser with their corresponding laser currents.

\begin{tabular}{|c|l|l|l|l|l|}
\hline$\lambda$ & $\Delta v$ & fm & bm & phase & soa \\
\hline $1523 \mathrm{~nm}$ & $50 \mathrm{MHz}$ & 13016 & 49647 & 11712 & 14657 \\
\hline $1525 \mathrm{~nm}$ & $50 \mathrm{MHz}$ & 4173 & 22664 & 10276 & 13119 \\
\hline $1529 \mathrm{~nm}$ & $75 \mathrm{MHz}$ & 14717 & 39474 & 8864 & 12563 \\
\hline $1534 \mathrm{~nm}$ & $75 \mathrm{MHz}$ & 22116 & 45904 & 7610 & 11932 \\
\hline
\end{tabular}




\begin{tabular}{|l|l|l|l|l|l|}
\hline $1539 \mathrm{~nm}$ & $50 \mathrm{MHz}$ & 34736 & 53504 & 16493 & 11849 \\
\hline $1545 \mathrm{~nm}$ & $50 \mathrm{MHz}$ & 39503 & 46982 & 12729 & 11218 \\
\hline $1546 \mathrm{~nm}$ & $50 \mathrm{MHz}$ & 27048 & 31647 & 13278 & 10796 \\
\hline $1551 \mathrm{~nm}$ & $60 \mathrm{MHz}$ & 45088 & 39538 & 9810 & 10567 \\
\hline $1557 \mathrm{~nm}$ & $65 \mathrm{MHz}$ & 52534 & 35049 & 16698 & 10696 \\
\hline $1564 \mathrm{~nm}$ & $100 \mathrm{MHz}$ & 39771 & 21950 & 19923 & 10987 \\
\hline
\end{tabular}

Further observation of the currents that make a wider linewidth as seen in Table 5 , seem to indicate no obvious trend. A visual representation of these currents can be found in Figure $3-13$.

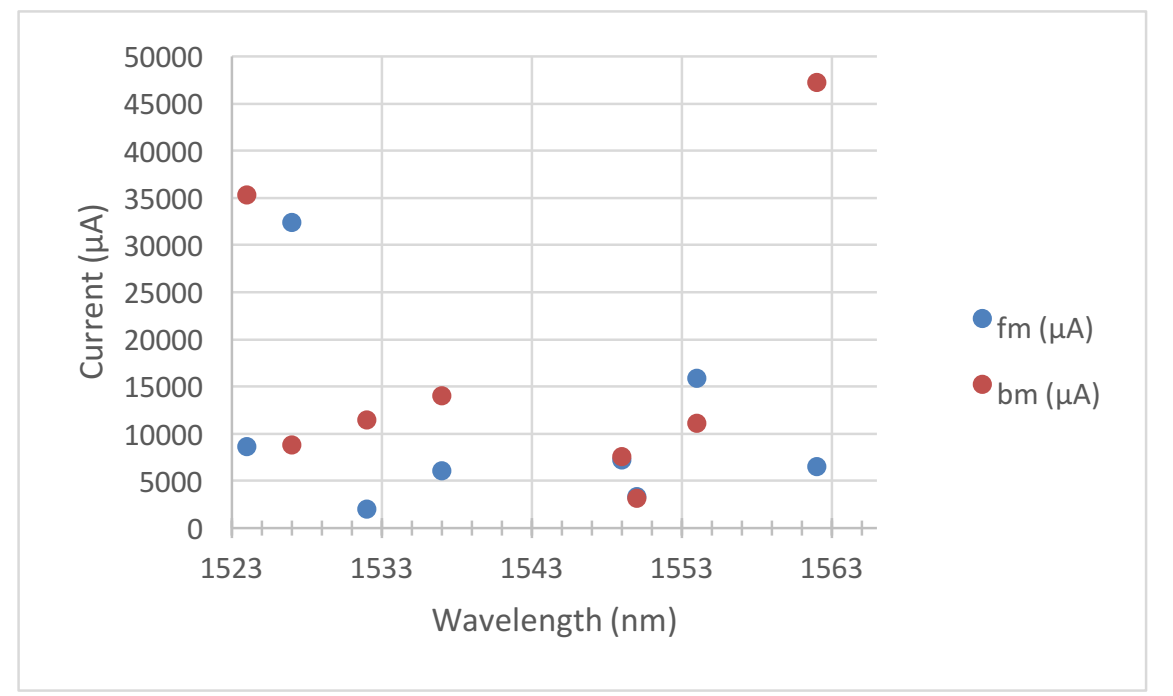

Figure 3-13 - Front mirror (fm) and back mirror (bm) currents at linewidth peaks across the tested wavelength range.

However, further observation of the currents that make a narrower linewidth as seen in Table 6 , seem to indicate a somewhat vague trend, but a trend nonetheless. In the lower end of the wavelength range, there is lower front mirror 
current then back mirror current. Around the middle of the wavelength range, there is closer to equal front mirror and back mirror current. At the higher range of the wavelength range, the front mirror current is higher than the back mirror current. The trend can be seen in Figure 3-14. The linear fit shown in Figure 3-14 shows the general trend but in no way indicates a good fit in which a mathematical model can be derived.

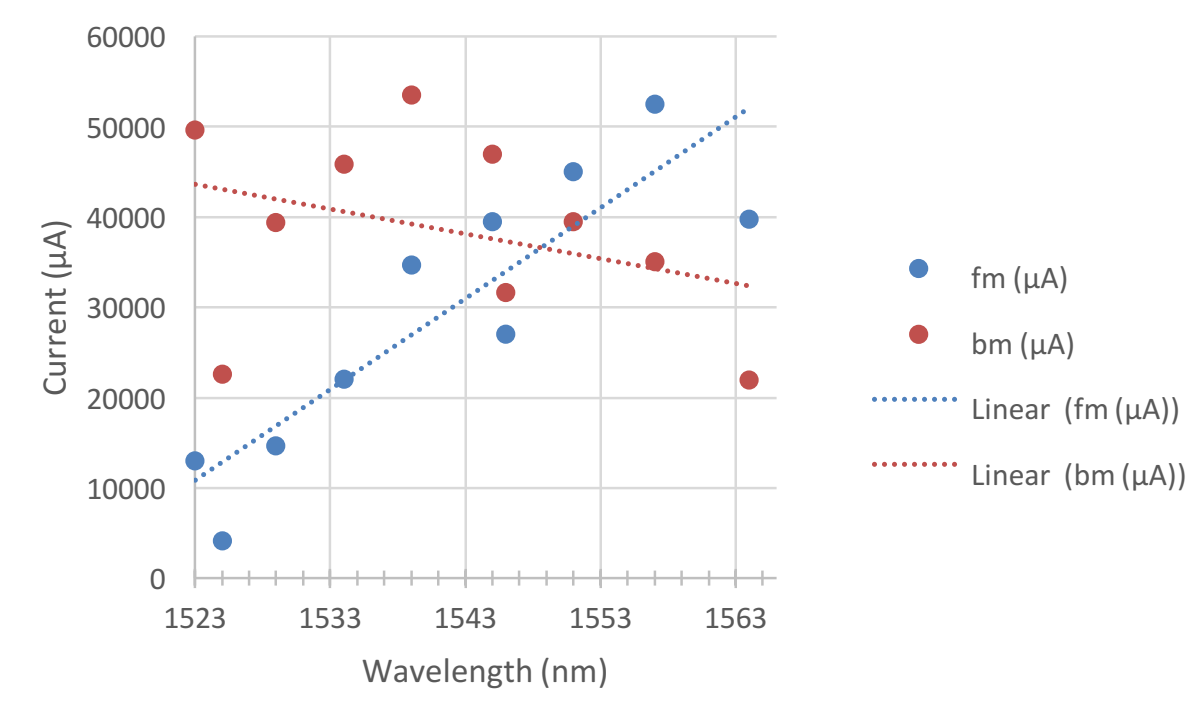

Figure 3-14 - Trend of the front mirror (fm) and back mirror (bm) currents at linewidth troughs across the tested wavelength range.

With a proposed trend for narrower linewidths as seen in Figure 3-14, the front mirror currents of peak and trough linewidths can be plotted on one graph to see how much the front mirror currents at a wider linewidth deviate from the trend and this can be seen in Figure 3-15. This 
can be done with the back mirror currents as well and can be seen in Figure 3-16. Although plotting on one graph might be helpful in order to see how both the front mirror and back mirror currents at a certain wavelength comply with their respective trends, they were decided to be graphed separately in order to keep the graphs less difficult to read.

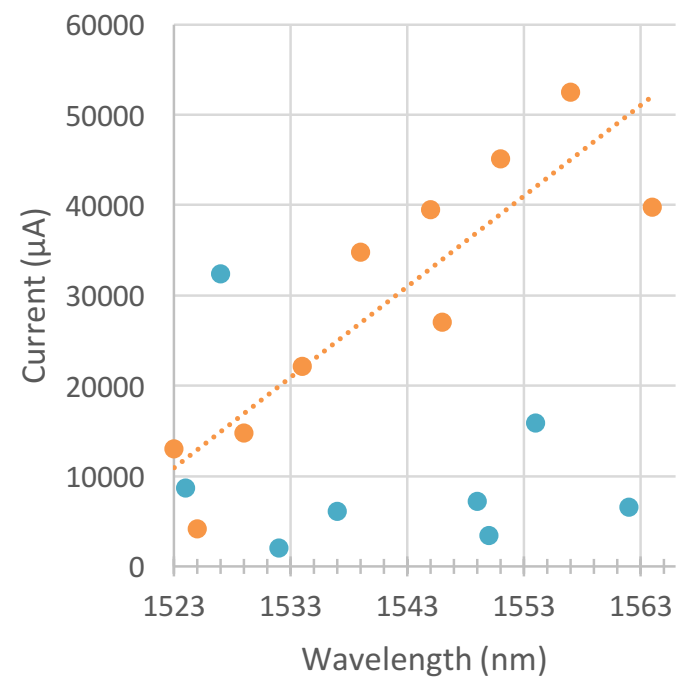

trough $\mathrm{fm}(\mu \mathrm{A})$

- peak $\mathrm{fm}(\mu \mathrm{A})$ Linear (trough $\mathrm{fm}(\mu \mathrm{A})$ )

Figure 3-15 - Trough front mirror (trough fm) and peak front mirror (peak $\mathrm{fm}$ ) currents plotted on same graph to highlight deviation from proposed trend. 


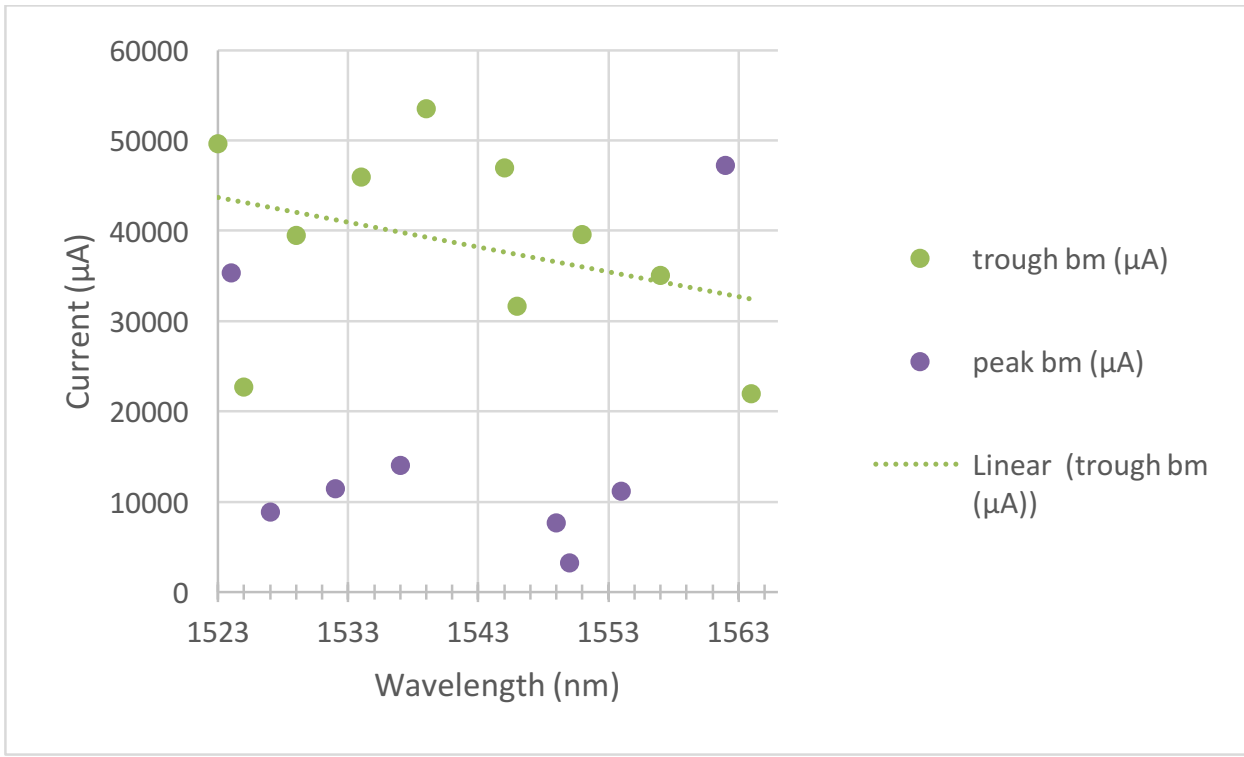

Figure 3-16 - Trough back mirror (trough bm) and peak back mirror (peak bm) currents plotted on same graph to highlight deviation from proposed trend.

As seen in both Figure $3-15$ and $3-16$, there is indication that the peak front mirror and back mirror currents deviate from the proposed trend. However, upon further observation it can be seen that there is one wavelength that seems to contradict the trend. The front mirror and back mirror currents at the wavelength of 1524 $\mathrm{nm}$ hug the respective proposed trends closely but has been labeled as a peak linewidth. Upon further investigation, using Table 5 as a reference, the linewidth at that wavelength is $85 \mathrm{MHz}$. It was labeled as a peak linewidth due to the lower linewidth on each side of it, which was a linewidth of $50 \mathrm{MHz}$ at 1523 and $1525 \mathrm{~nm}$. Thus an argument for the original proposed trend can be that although in the data set that was taken, the $85 \mathrm{MHz}$ is labeled as a peak, 
it is not technically a high linewidth when compared against the other linewidths found in the range under test. Moving on to more of the rest of the lineshape, upon initial observation of the captured tabulated results regarding lineshape as can be found in Appendix B, the spectral shapes of the various wavelengths seem to expectedly follow a pattern in which a narrower linewidth, defined as $3 \mathrm{~dB}$ down from peak $\Delta v$, leads to a narrower width from $0 \mathrm{~Hz}$ at $10 \mathrm{~dB}$ down $\Delta v_{10 d B}, 20 \mathrm{~dB}$ down $\Delta v_{20 d B}$, and $30 \mathrm{~dB}$ down $\Delta v_{30 d B}$ from peak. What is interesting however is the difference in lineshapes even though the $\Delta v^{\prime}$ s are the same. For example, $1523 \mathrm{~nm}$ and $1525 \mathrm{~nm}$ have an equivalent linewidth of $50 \mathrm{MHz}$, but the lineshape is noticeably different between the two wavelengths. This is further highlighted quantitatively in Figure 3-17 along with the rest of the wavelengths across the range. 


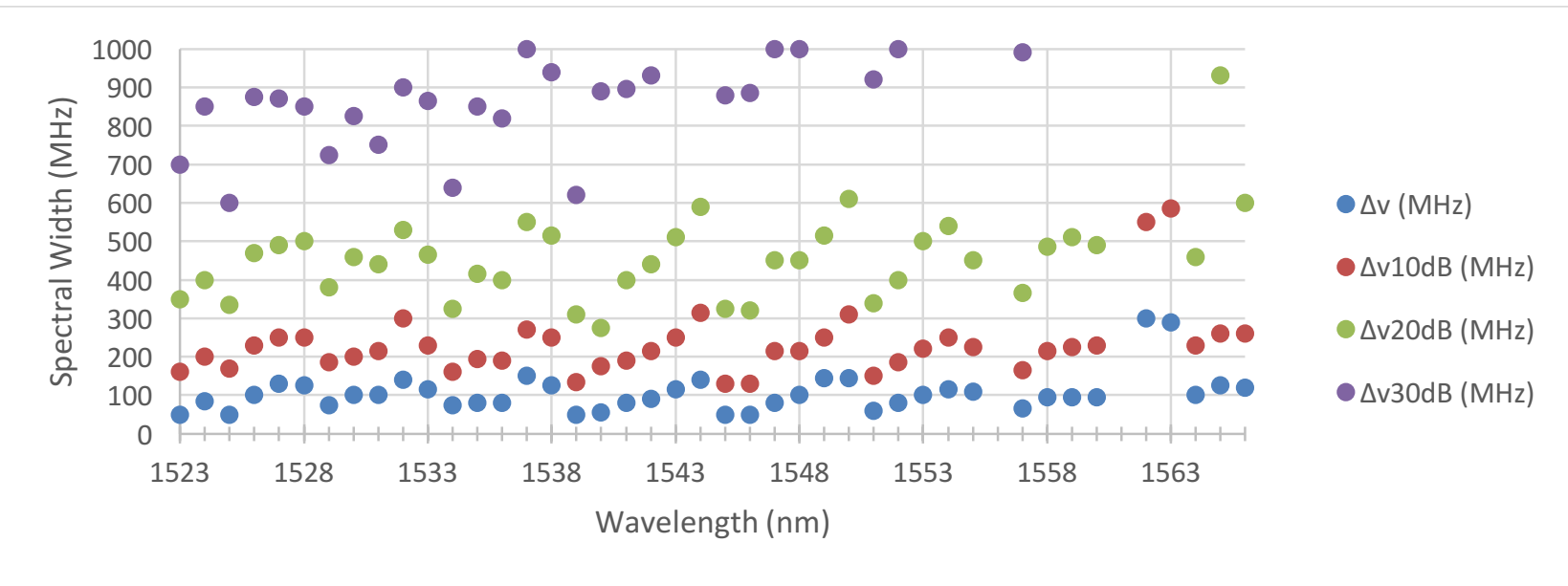

Figure 3-17 - Frequencies at $3 \mathrm{~dB}(\Delta v), 10 \mathrm{~dB}\left(\Delta v_{10 d B}\right), 20 \mathrm{~dB}$ $\left(\Delta v_{20 d B}\right)$, and $30 \mathrm{~dB}\left(\Delta v_{30 d B}\right)$ down from peak for wavelengths across the range of 1523 to $1566 \mathrm{~nm}$.

Regarding the comparison of the experimental

lineshapes to the theoretical Lorentzian lineshape values found in Appendix $B$, there is a large difference especially when comparing the frequencies at $\Delta v_{20 d B}$ and $\Delta v_{30 d B}$. The lineshape found in this experiment is much narrower at these points than the theoretical Lorentzian values, and is more similar to a Gaussian shape. A Gaussian and Lorentzian comparison is visually illustrated in Figure 3-18. 


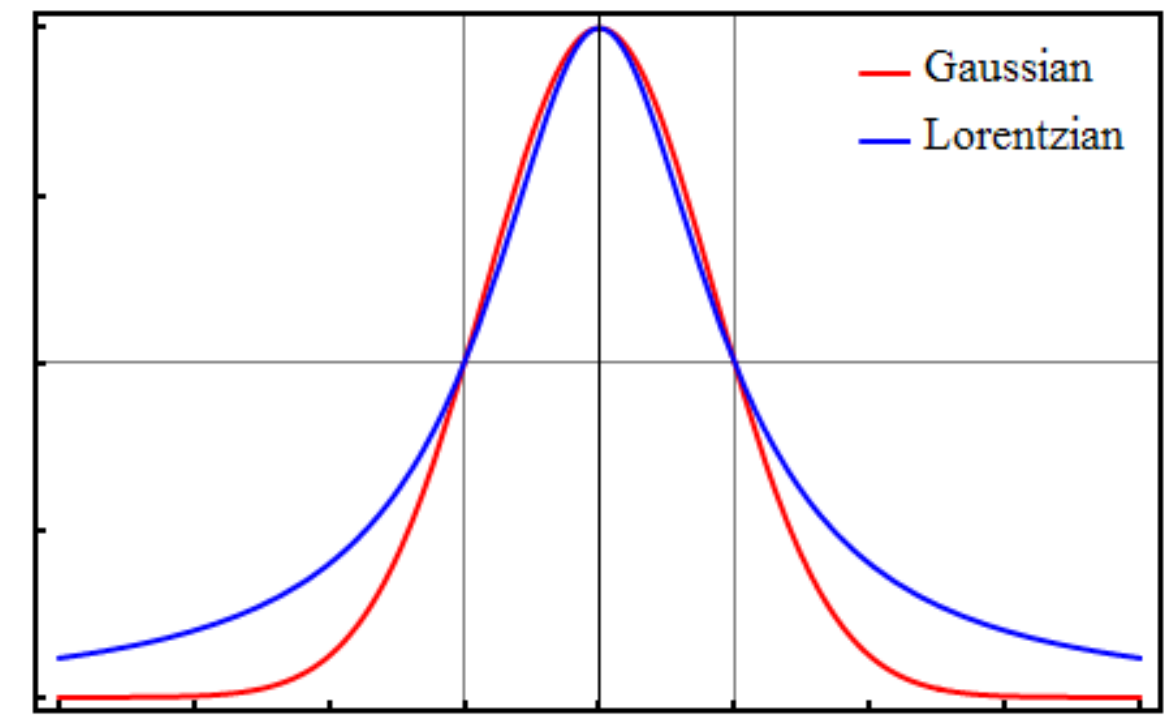

Figure 3-18 - Lorentzian spectral shape and a Gaussian spectral shape overlaid [23]. 


\section{Chapter 4. Ranging with Interference Fringes}

With investigation into the linewidths of the wavelengths across the Insight laser output range of 1523 to $1566 \mathrm{~nm}$, coherence times and coherence lengths were calculated for fixed wavelengths as can be seen in Appendix A. In order to test the laser's ability to abide by those calculations, but more importantly, get a general trend for how effective the laser might be at ranging, an interference fringe test was done. For initial test purposes, the laser was chosen to be swept across the entire wavelength range, 1522.13 to $1566.18 \mathrm{~nm}$, that was available to be output by laser in the fixed wavelength mode as seen in Section 3.1 of Chapter 3, in order to keep the two tests consistent with each other so that comparisons can be drawn.

\subsection{Interference Fringe Test Setup and Theory}

With the Insight Laser Control Program the laser can be set in swept wavelength mode. The interface is shown in Figure 4-1. 


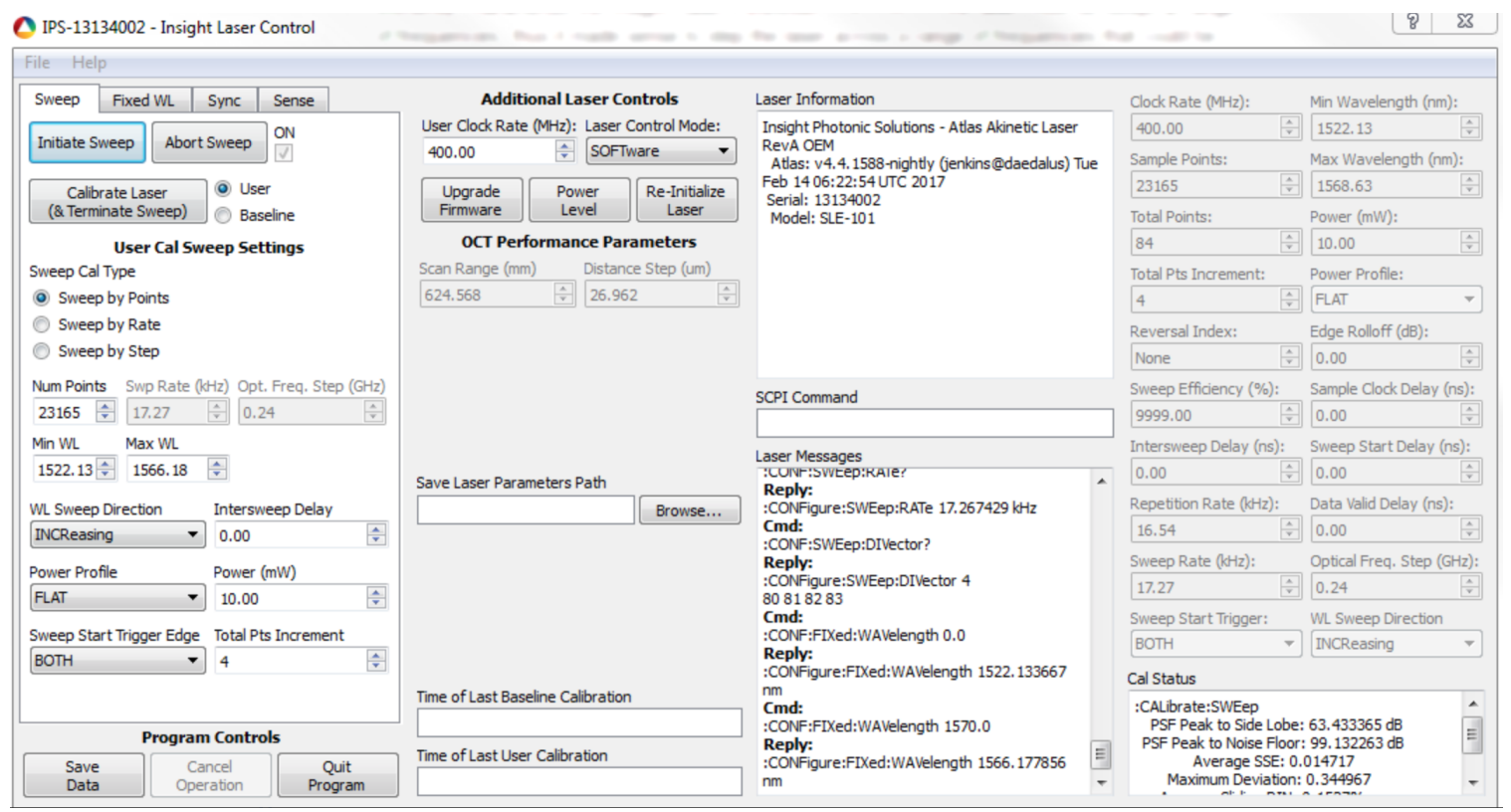

Figure 4-1 - Insight Laser Control Program swept wavelength output interface [20].

The swept wavelength interface of this laser allows the user to sweep across a chosen range of frequencies between $1522.13 \mathrm{~nm}$ and $1566.18 \mathrm{~nm}$ with modifiable power and various other modifiable characteristics of the sweep as shown in Figure 4-1. The output of the laser for this test is a wavelength sweep across the entire range from 1522.13 $\mathrm{nm}$ to $1566.18 \mathrm{~nm}$ and at a power of $0 \mathrm{dBm}$ which is shown in a Optical Spectrum Analyzer (Agilent 86140B) to visually highlight the characteristics of the laser in the frequency spectrum in Figure 4-2. 


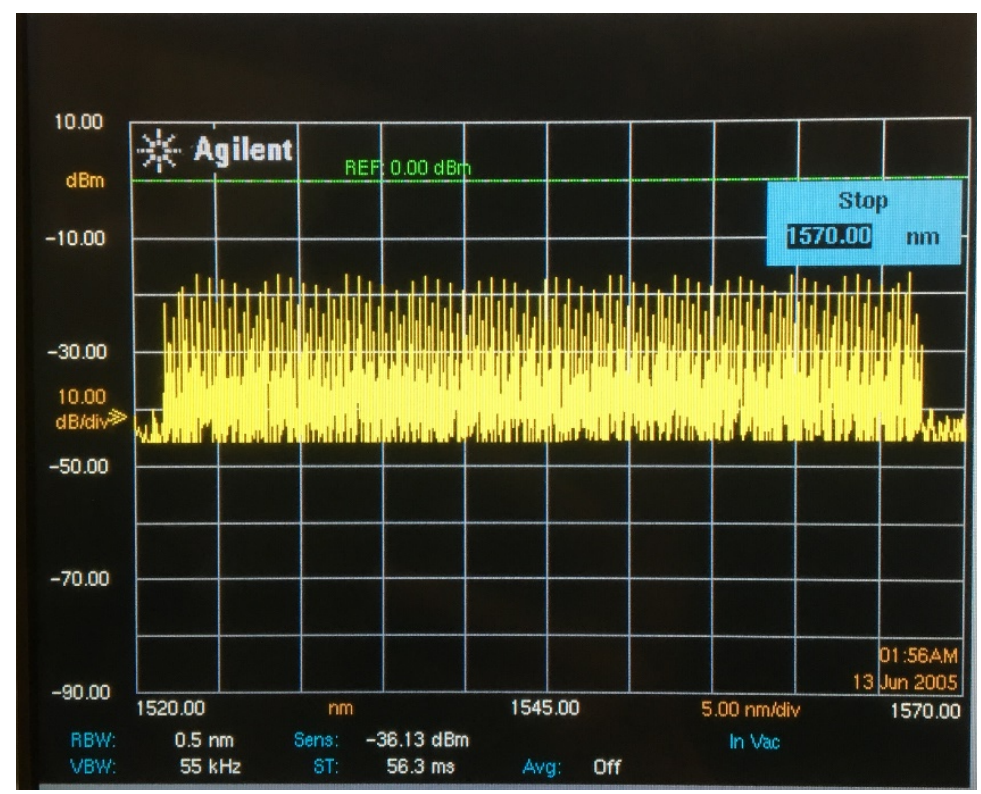

Figure 4-2 - Swept $1522.13 \mathrm{~nm}$ to $1566.18 \mathrm{~nm}$ output of laser shown in optical spectrum analyzer.

With the laser under test settings determined, the next step was to determine the method of actually obtaining the interference fringes in order to use them to test the ranging capabilities of the swept laser. Luckily, the method of obtaining these interference fringes isn't too vastly different from obtaining linewidth.

As illustrated in Section 3.2 of Chapter 3, to obtain linewidth, an interferometer was used to split the laser source outputting a fixed wavelength into two different paths only to be brought together before being sent into a photodetector to be converted into electrical signals for the electrical spectrum analyzer. The interferometer used in that experiment had a very large delay of $3.5 \mu$ s in one path compared to the other path. 
In order to obtain interference fringes, the setup is almost the same except the laser source is swept across a range of frequencies and is split into two paths with a much smaller path length difference. For ranging purposes, this path length difference would be the distance one would hope to measure.

Qualitatively explained, the laser is sweeping frequencies linearly across a range, just like a radar would in FMCW. Recalled from before in section 1.2 of Chapter 1 , the radar in FMCW will be able to determine the distance between itself and an object, knowing when it sent out a certain frequency in its sweep and being able to calculate the time difference when it detects that certain frequency at the receiver. With that time difference it is able to calculate distance knowing the speed of its signals. The concept of this interference fringe test for the laser is similar in that the sweeping laser source is split into two different paths by the interferometer. There will be a path length difference, so one path will have a delay. Comparing to the radar FMCW concept, the shorter path can be seen as the frequency that is being sent out by the transmitter, and the longer path can be seen as frequency that is being sent to the receiver after reflecting off the object to be detected. After being split 
by the interferometer and traveling through different path lengths, this will lead to two different frequencies, due to the sweep, coming together at the end of the interferometer to combine with each other constructively and destructively, which will create interference fringes. The spacing between the interference fringe peaks will indicate the delta wavelength of the sweep $\Delta \lambda$, depending on the path length difference $\Delta L$, and knowing the velocity of light in optical fiber $v_{g}$, this path length difference can be found. How $\Delta \lambda$ is related to interference fringes is illustrated in Figure 4-3.

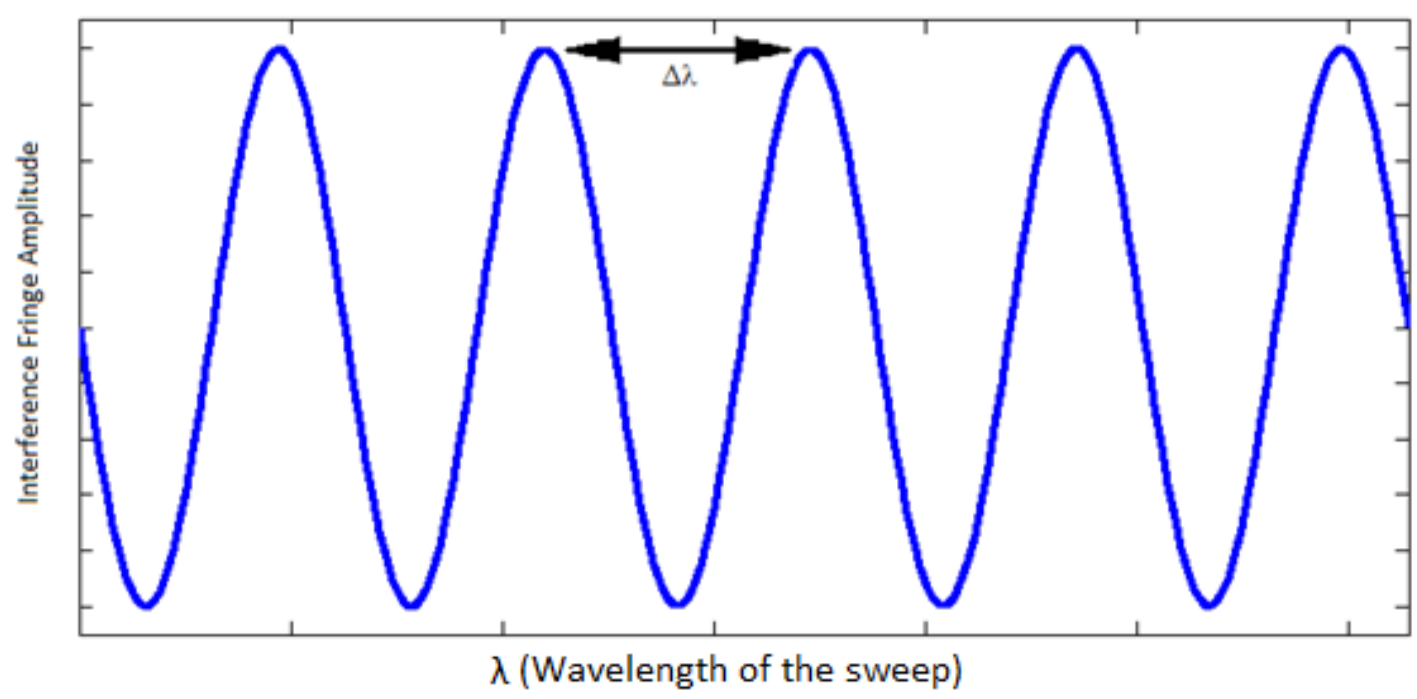

Figure 4-3 - The delta wavelength of the sweep $\Delta \lambda$ is the difference in the sweep of the laser between two peaks of the interference fringes. 
The delta wavelength of the sweep $\Delta \lambda$ decreases with a $\Delta L$ increase of the interferometer. The rate at which $\Delta \lambda$ decreases is determined by the laser linewidth. A narrower laser linewidth will allow a larger $\Delta L$ (because the maximum $\Delta L$ is determined by the coherence length), before the $\Delta \lambda$ decreases to a point where peaks cannot be determined as the wavelengths combine completely destructively at the output of the interferometer. Figure 4-4 provides a visual representation of how interference fringes can change over increasing path length differences $\Delta L$. 
(a)

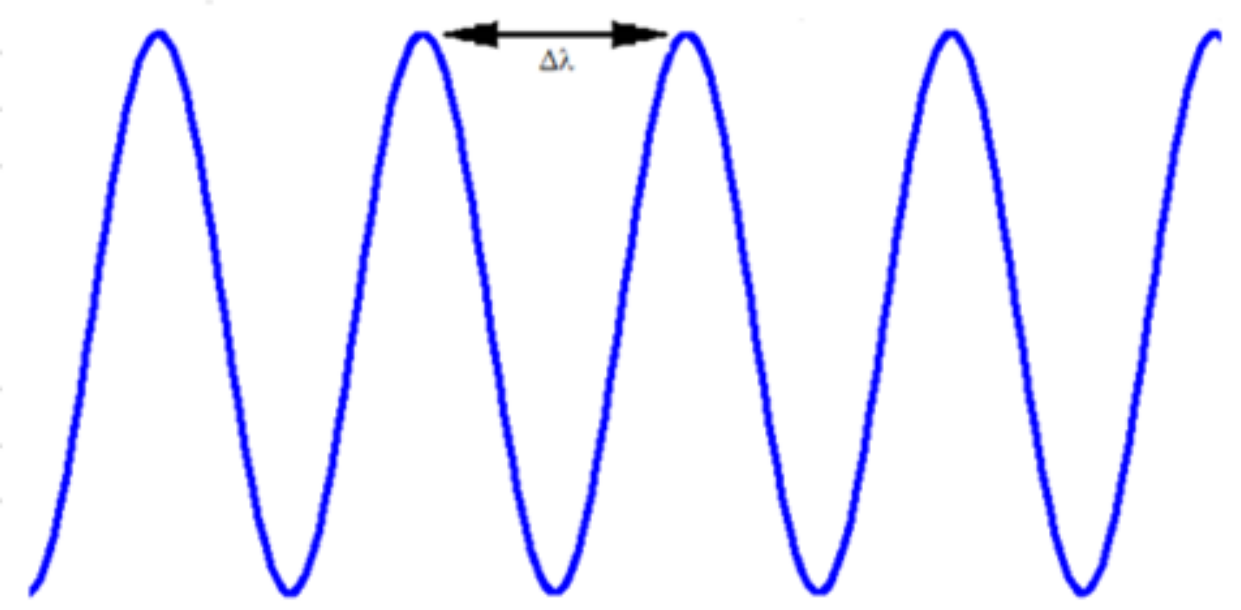

(b)

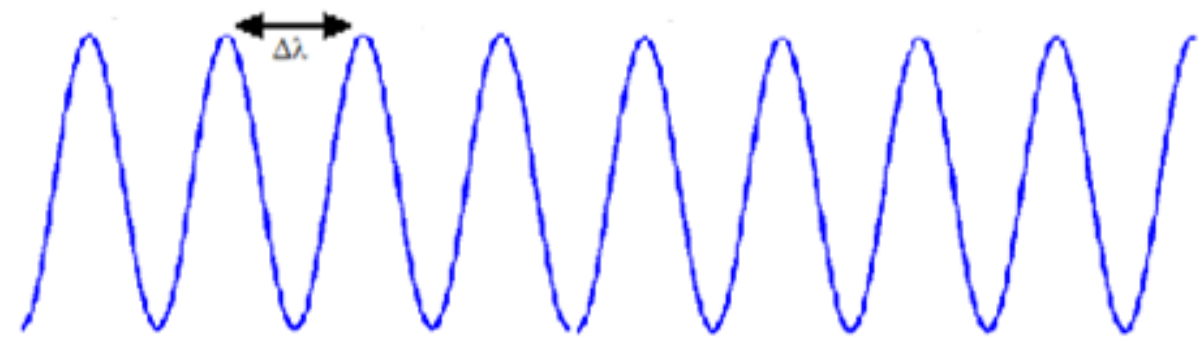

(c)

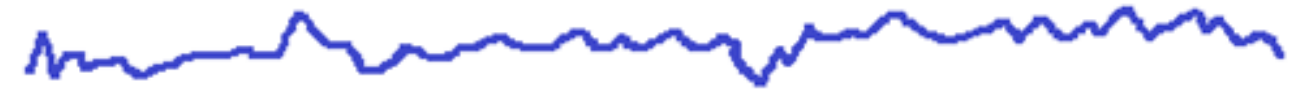

$\lambda$ (Wavelength of the sweep)

Figure 4-4 - Example of time domain interference fringes at (a) a small $\Delta L$ (b) a medium $\Delta L$ (c) a large $\Delta L$.

Quantitatively explained, one can refer to the

derivation of the relationship between $\Delta \lambda$ or delta frequency of the sweep $\Delta f$ (known as the free spectral range of the interference fringes), and $\Delta L$ for a Mach-Zehnder interferometer, which is what was used for the linewidth measurements in Chapter 3, and is what is essentially created in the interference fringe test. In the Mach- 
Zehnder, the laser is split into two different path lengths at one end and is joined together at the other end, and because the laser is sweeping, the phases are different as can be illustrated in Figure 4-5.

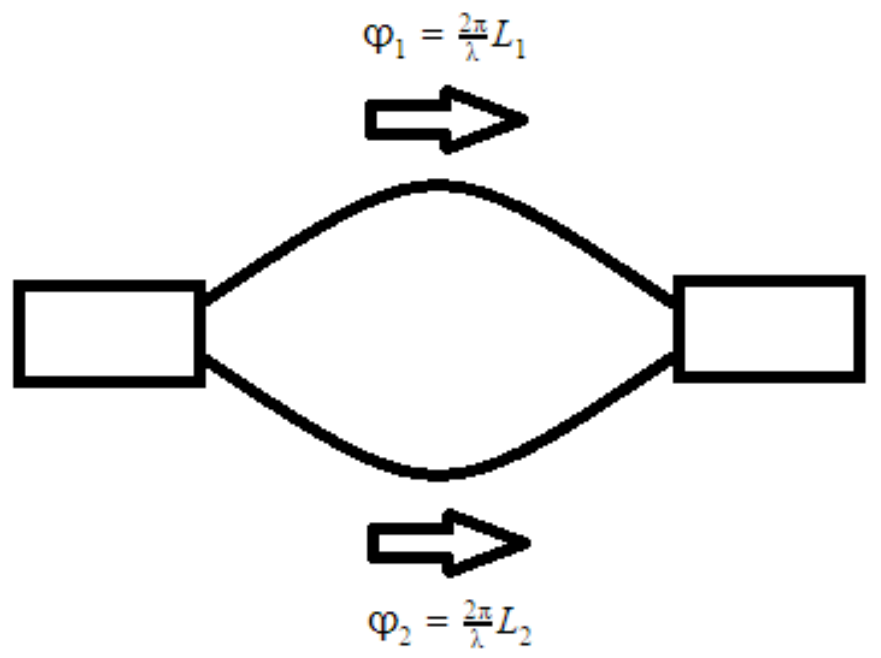

Figure 4-5 - Phase and length relationship across two different lengths.

Taking into account the phase relationship with length, and how the phase changes with a sweeping wavelength, useful equations which will allow one to find path length difference from $\Delta \lambda$ or $\Delta f$ is derived as follows:

$$
\begin{gathered}
\phi_{1}=\frac{2 \pi}{\lambda} L_{1} \\
\phi_{2}=\frac{2 \pi}{\lambda} L_{2} \\
\frac{\delta \phi_{1}}{\delta \lambda}=\frac{-2 \pi L_{1}}{\lambda^{2}} \\
\frac{\delta \phi_{2}}{\delta \lambda}=\frac{-2 \pi L_{2}}{\lambda^{2}}
\end{gathered}
$$




$$
\begin{gathered}
\frac{\Delta \phi}{\Delta \lambda}=\frac{\delta \phi_{1}}{\delta \lambda}-\frac{\delta \phi_{2}}{\delta \lambda}=\frac{2 \pi \Delta L}{\lambda^{2}} \text { where } \Delta L=\left|L_{1}-L_{2}\right| \\
\frac{2 \pi}{\Delta \lambda}=\frac{2 \pi \Delta L}{\lambda^{2}} \\
\text { group velocity index } n_{g}=1.47 \text {, for optical fiber } \\
\text { velocity of light within fiber } v_{g}=\frac{c}{n_{g}} \\
\Delta \lambda=\frac{\lambda^{2}}{\Delta L}=\frac{v_{g}}{f_{1}}-\frac{v_{g}}{f_{2}}=\frac{v_{g} \Delta f}{f^{2}} \\
\frac{\lambda^{2}}{\Delta L}=\frac{v_{g} \Delta f}{f^{2}} \\
\frac{\left(v_{g} / f\right)^{2}}{\Delta L}=\frac{v_{g} \Delta f}{f^{2}} \\
\Delta L=\frac{v_{g}}{\Delta f}
\end{gathered}
$$

The derivation can be summarized in three very useful equations :

$$
\begin{gathered}
\text { Free Spectral Range FSR }=\Delta f=\frac{v_{g}}{\Delta L}=v_{g} \frac{\Delta \lambda}{\lambda^{2}} \\
\Delta \lambda=v_{g} \frac{\Delta f}{f^{2}} \\
\Delta L=\frac{v_{g}}{\Delta f}
\end{gathered}
$$

The equations above allows one to obtain the path length difference from the free spectral range of the interference fringes and the velocity of light in optical fiber.

The test setup to obtain interference fringes is very similar to how the linewidth test was setup in Section 3.2 
of Chapter 3 except that these Mach-Zehnder type interferometers had to be made with much smaller path length differences then was presented in the off the shelf module that was used for the linewidth tests which had a $3.5 \mu$ delay. Because there was no off the shelf module available for path length differences which would successfully be able to test the relatively short path length difference as indicated by the range of coherence lengths as shown in Appendix A, the interferometers with these path length differences were made. Two 1x2 couplers (Thorlabs TW1550R5A1) with a theoretical splitting ratio of $50 / 50$ (50\% one arm and $50 \%$ on the other) were used. These couplers had a bandwidth of $1550 \mathrm{~nm} \pm 100 \mathrm{~nm}$, which was perfect for the output wavelength range of this laser of $1522.13 \mathrm{~nm}$ to $1566.18 \mathrm{~nm}$. With FC APC male connectors already available on the inputs and outputs of the couplers, interchanging lengths in between them was simply done using FC APC male to male adapters. The couplers and the adapters in the test layout can be found in Figure 4-6. 


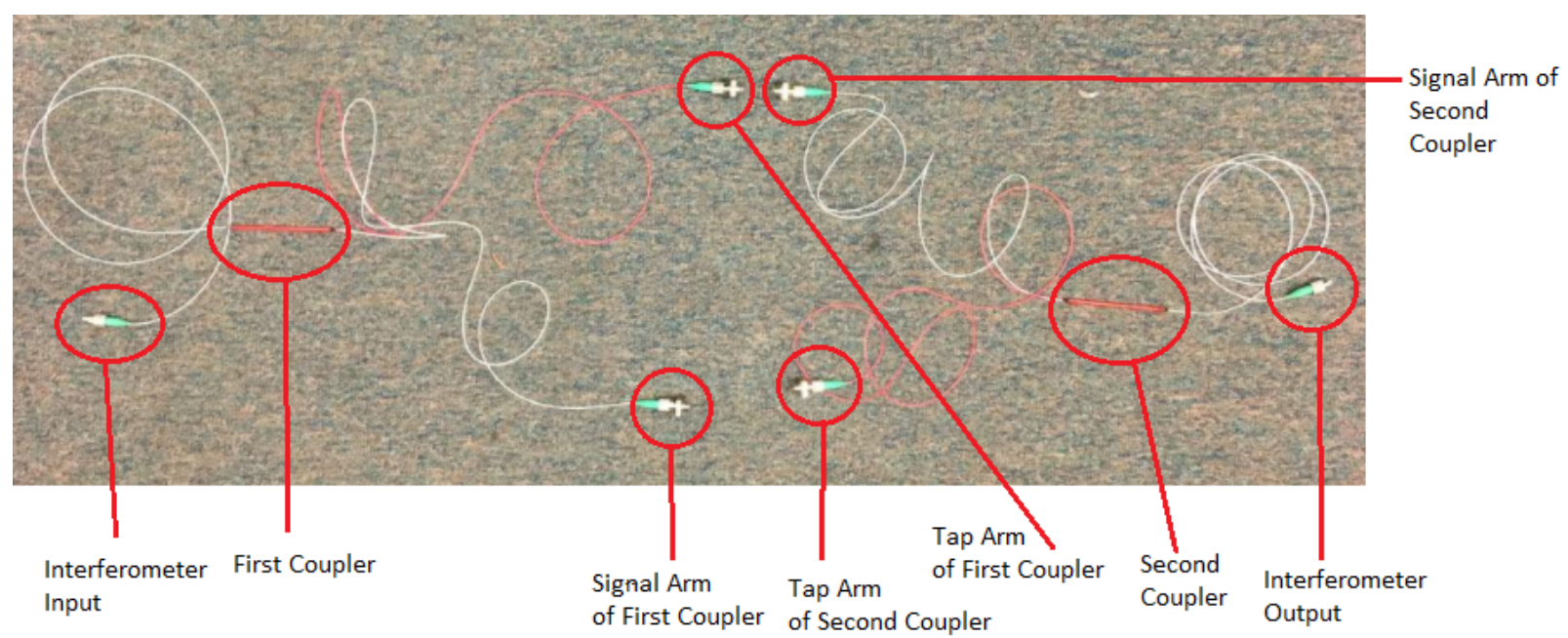

Figure 4-6 - Homemade Mach-Zehnder interferometer allowing for interchangeable lengths.

A polarization controller was also made to be able to plug into the homemade interferometer. Although light waves are able to vibrate in multiple planes, a polarization controller allows one to tune light waves to vibrate in one plane, which is preferable for consistency in test. The light directly output by the laser is polarized; however, the optical fiber is birefringent which alters the polarization. In order to try and reach maximum polarization, but more importantly, avoid destructive polarization differences when the two lights combine at the end of the interferometer with the second coupler, a polarization controller was made with a cardboard backplane. The dimensions and number of rotations were based off a 2 paddle polarization controller. This homemade polarization controller can be found in Figure 4-7. 


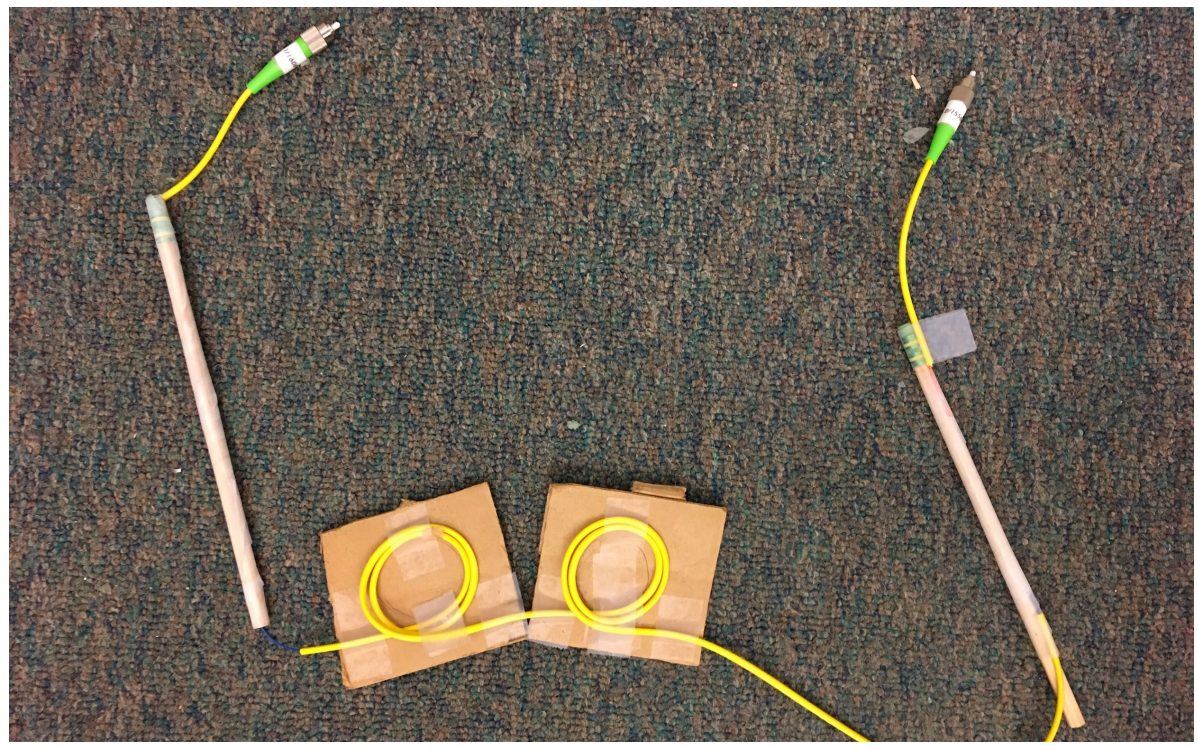

Figure 4-7 - Homemade two paddle polarization controller.

\subsection{Interference Fringe Test}

With a basic theoretical background and a method of capturing path length difference determined, the experimental setup to capture the interference fringes of swept wavelengths across the wavelength range able to be output by the Insight laser at different path length differences was begun.

The laser is connected to the homemade interferometer shown in Figure 4-7 and a variable path length difference is inserted into the interferometer. The output of the interferometer is plugged into an off the shelf photodetector (Insight BPD-1). The output of that photodetector is then plugged into an oscilloscope (Tektronix TDS 784A or the 5105 LABVIEW oscilloscope setup) so that the interference fringes can be seen. A visual 
representation of the experimental setup for the interference fringe characterization of the Insight laser can be seen in Figure 4-8.

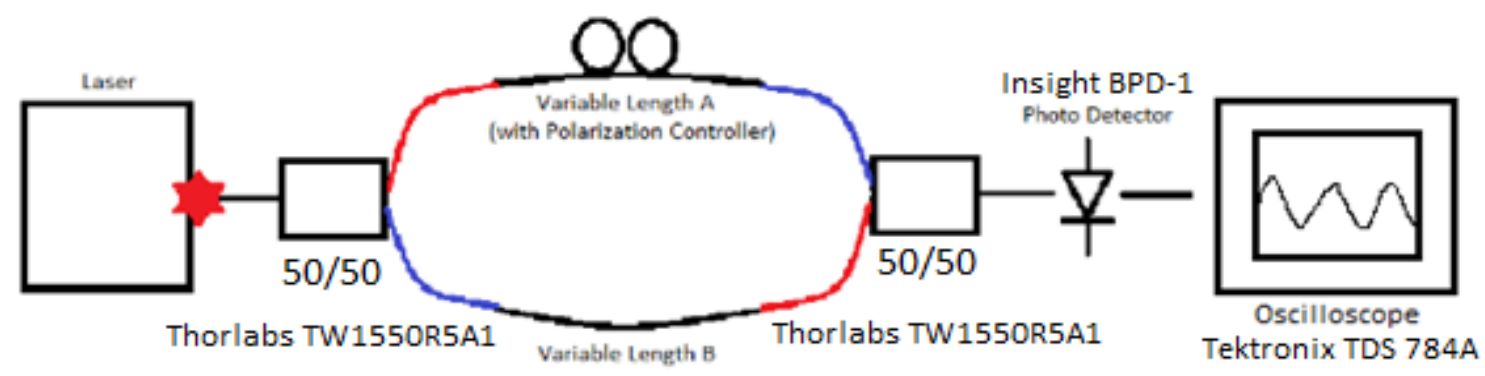

Figure 4-8 - Time domain interference fringe test setup.

Using the interference fringe test setup as seen in Figure 4-8, 9 different path length differences were inserted. This ranged from very small ruler measured path length differences of $<1 \mathrm{~cm}$ (no observable measurable path length difference) to relatively larger ruler measured path length differences such as $187.5 \mathrm{~cm}$. Figure 4-9 shows the interference fringes for a ruler measured path length difference of $<1 \mathrm{~cm}$ across the time scale for the laser to perform approximately 3 sweeps on the LABVIEW oscilloscope via use of a 5105 PCI Digitizer ADC and a 5105 Acquisition program. As can be observed by utilizing Waveform Graph 2 of the program, the time from $1523.13 \mathrm{~nm}$ to $1566.18 \mathrm{~nm}$ is around $60 \mu s$. 


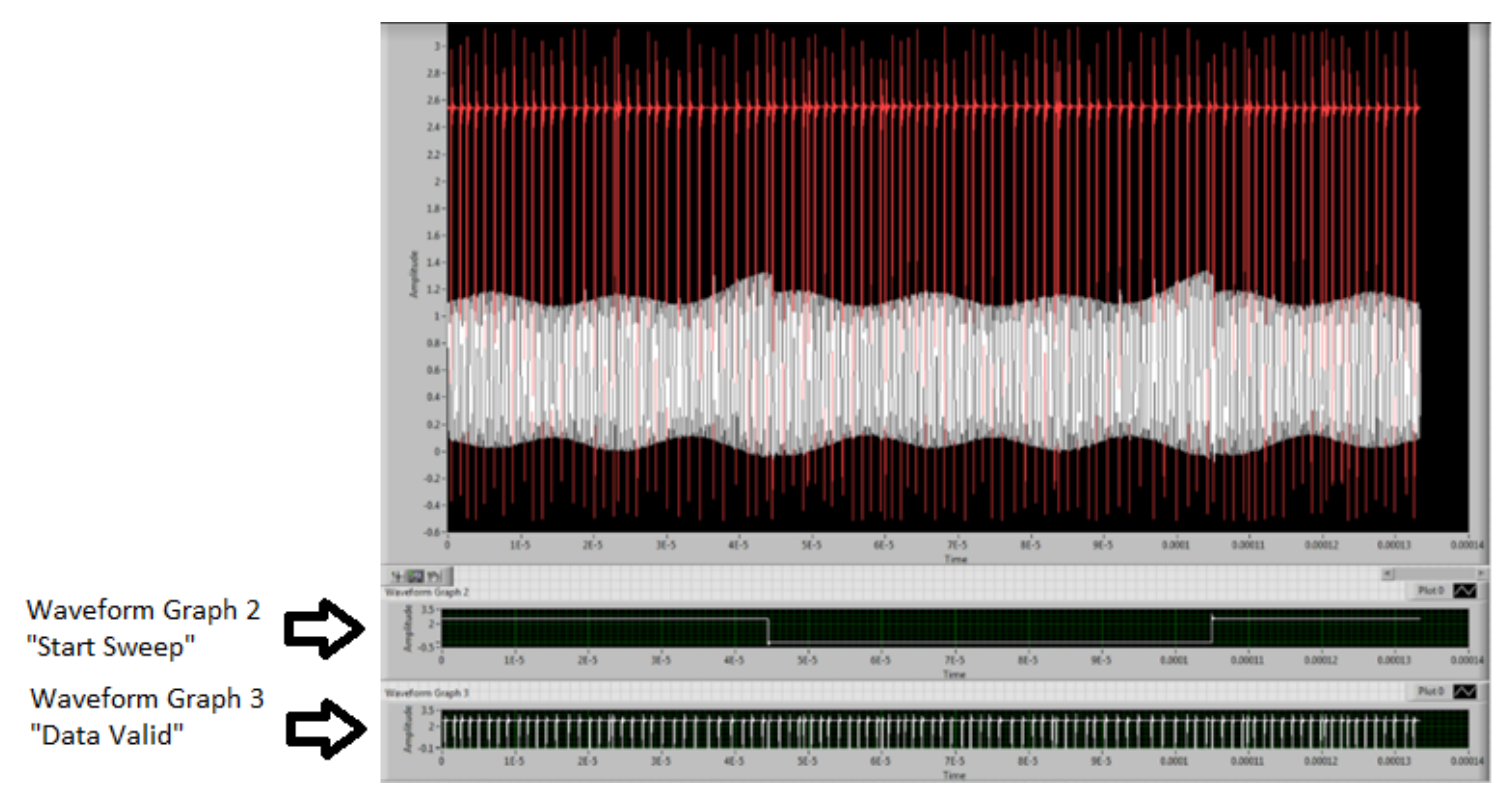

Waveform Graph 3 (zoomed)

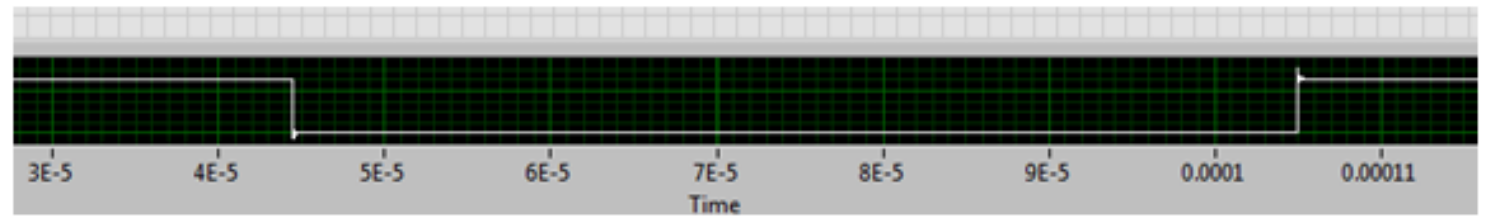

Figure 4-9 - Interference fringes as seen on 5105 Acquisition program across a time scale of around 3 laser sweeps for a ruler measured path length difference of $<1$ cm.

Scaled to a much smaller time scale so that the interference fringe frequency is more visible for all of the path length differences, and utilizing the high bandwidth Tektronix oscilloscope rather than the 5105 setup due to its limited bandwidth capabilities, please refer to Appendix $\mathrm{C}$ for the results of this experiment in tabular format. Figure 4-10 highlights the format in which the data was taken for example purpose for a ruler measured path length difference $\Delta L$ of $<1 \mathrm{~cm}$. 


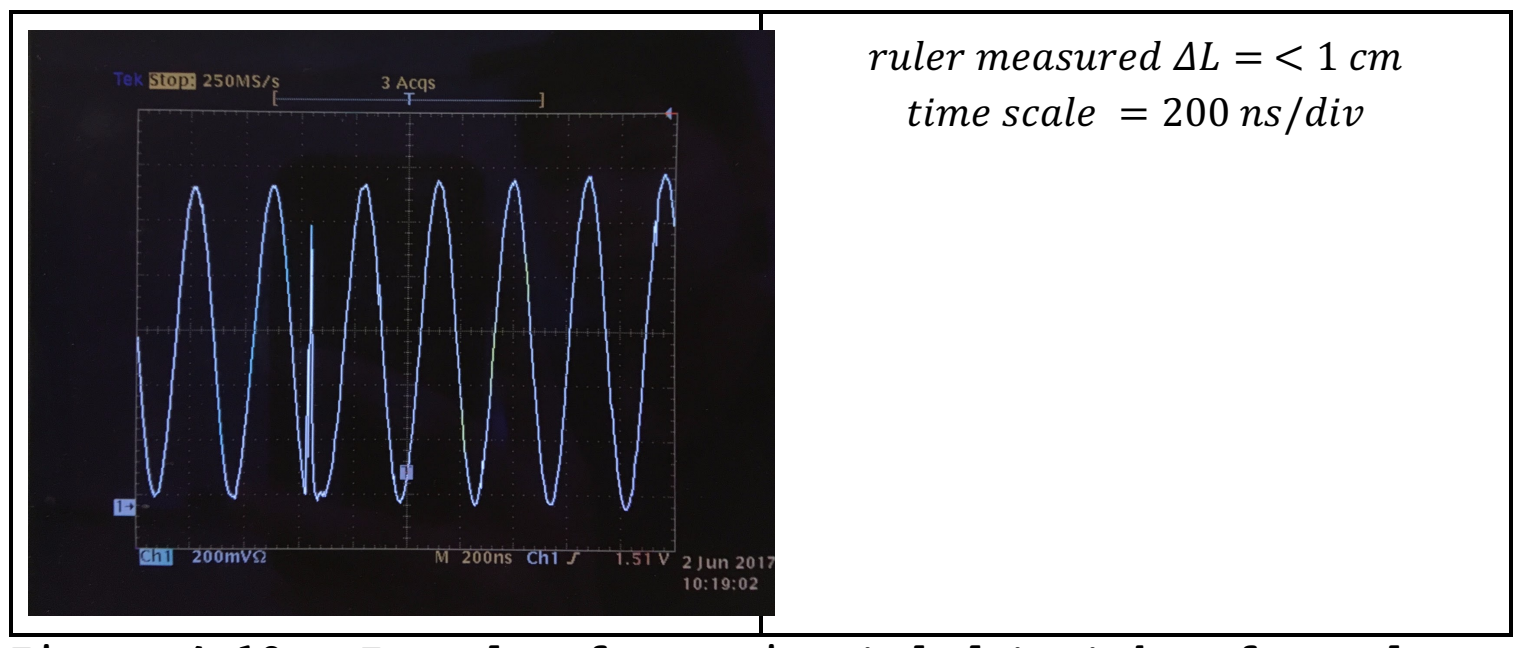

Figure 4-10 - Example of experimental data taken for ruler measured path length difference of $<1 \mathrm{~cm}$ in time domain interference fringe test.

With the time domain of the output of this experiment captured, in order to capture the frequency of these interference fringes $f_{i}$, a spectrum analyzer was utilized. This would not only allow one to capture the frequency of the interference fringes, but it would also allow one to observe the entire frequency spectrum and any harmonics that may present themselves. In order to do a frequency domain analysis, the output of the photodetector as seen in Figure 4-8 was connected to an off the shelf spectrum analyzer (Agilent Technologies N9000A). This frequency domain test setup can be seen in Figure 4-11. 


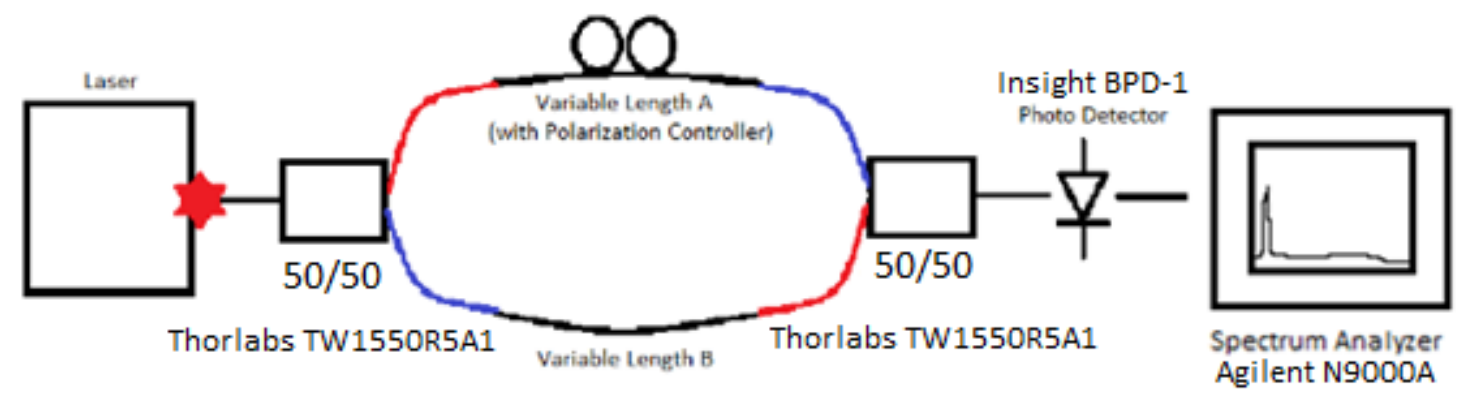

Figure 4-11 - Frequency domain interference fringe test setup.

The frequency domain test was done for all of the ruler measured path length differences that were tested in the time domain with the oscilloscope. The frequency at which there was peak power was recorded as the frequency of the interference fringe. Please refer to Appendix $D$ for the results of this experiment in tabular format. Figure 4-12 highlights the format in which the data was taken for example purpose for a ruler measured path length difference $\Delta L$ of $<1 \mathrm{~cm}$. 


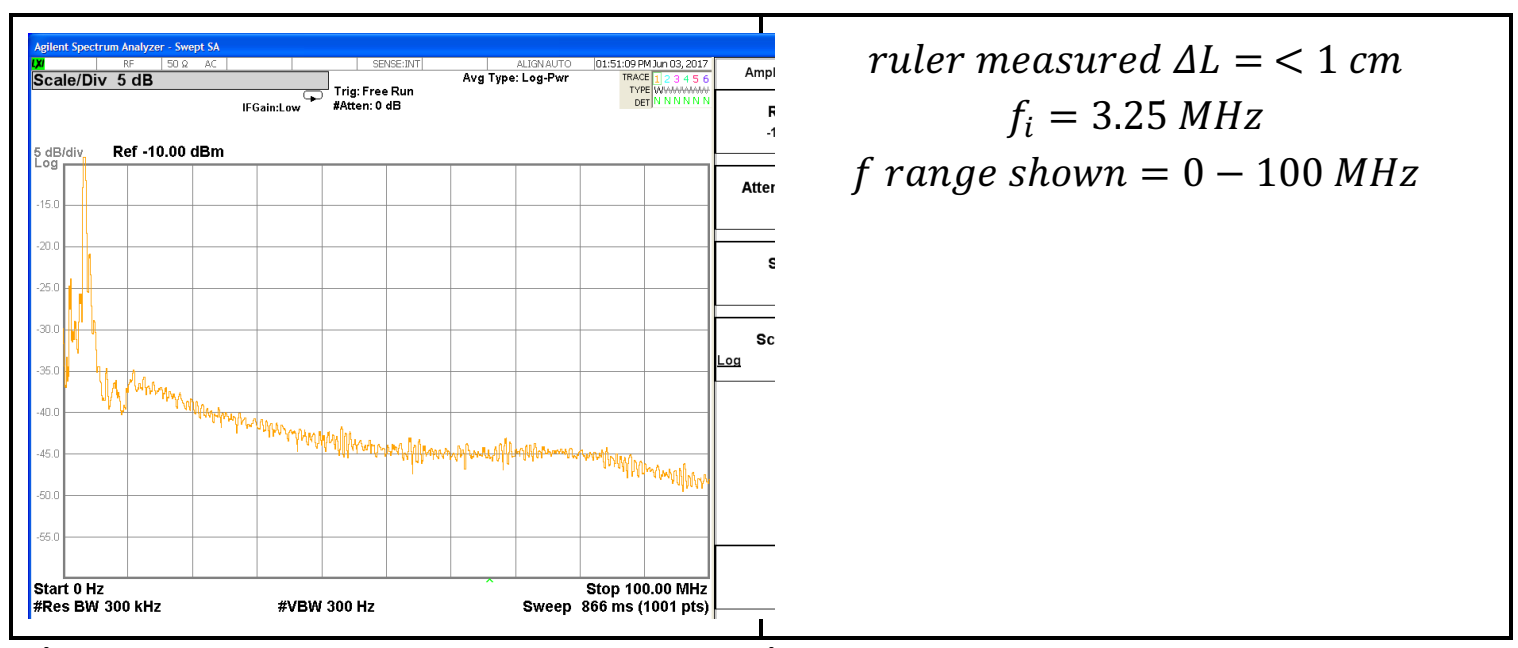

Figure 4-12 - Example of experimental data taken for ruler measured path length difference of $<1 \mathrm{~cm}$ in frequency domain interference fringe test.

\subsection{Interference Fringe Test Calibration Method}

With the equations necessary to find path length difference from the free spectral range $\Delta f$ or $\Delta \lambda$ from Section 4.1 , and the interference fringe time domain and frequency domain characteristics obtained from section 4.2, the next logical step would be to calculate exactly what path length differences are indicated by the interference fringes obtained and to see how well that lines up with the ruler measured path length differences.

In order to do this, the frequency of the interference fringes $f_{i}$ must be associated with $\Delta f$ or $\Delta \lambda$. This can be done a variety of ways including correlating the wavelength range of the sweep with the time it takes for one sweep, and calculating what sweep wavelength difference (or frequency difference) is correlated with a period of the 
interference fringes. Another way, similar to a sort of calibration, is to take a known value of $\Delta \lambda$ from an already tested interferometer and find the $f_{i}$ correlated with it. Because the time it takes for each laser sweep is constant, knowing how $\Delta \lambda$ and $f_{i}$ relate for one case will allow one to calculate the $\Delta \lambda$ of the rest of the cases, if given just $f_{i}$ of the other cases, by taking proportions.

Because there was an interferometer with a known $\Delta \lambda$ of $100 \mathrm{pm}$ available, the second calibration method was chosen. The output of the laser was connected to the input of the calibration interferometer, and the output of the interferometer was then connected to both the oscilloscope and spectrum analyzer. The output of the calibration interferometer with a $\Delta \lambda$ of $100 \mathrm{pm}$ in the time domain is shown in Figure 4-13, and the frequency domain of the same is shown in Figure 4-14. 


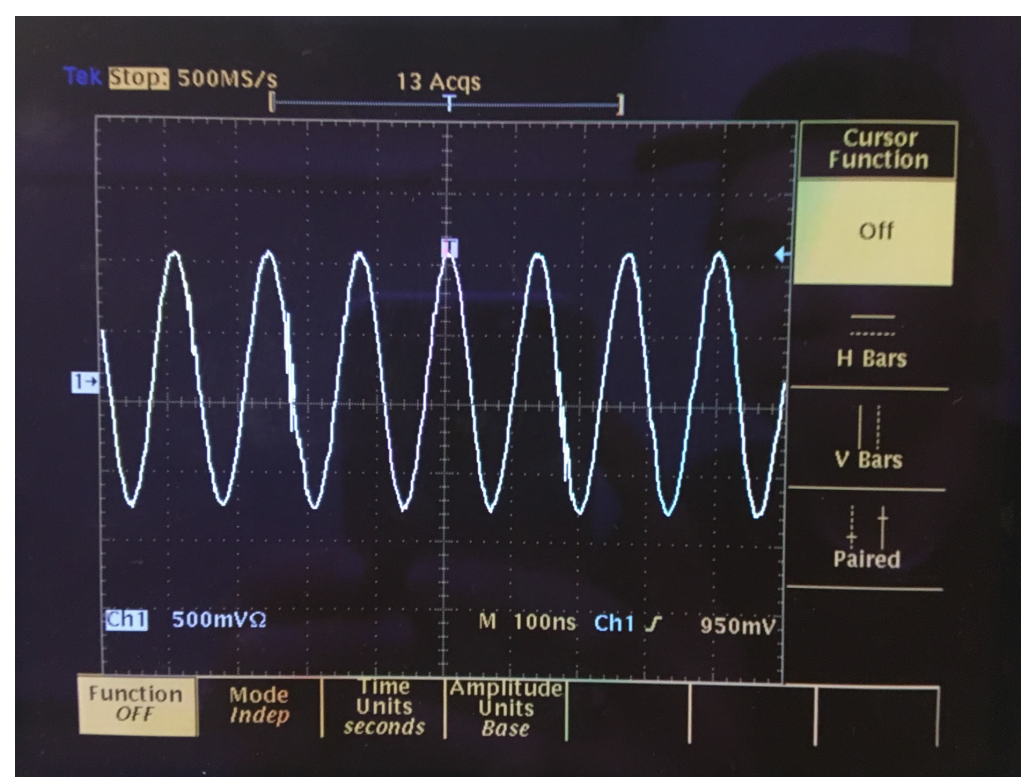

Figure 4-13 - Time domain output of calibration interferometer with known $100 \mathrm{pm} \Delta \lambda$.

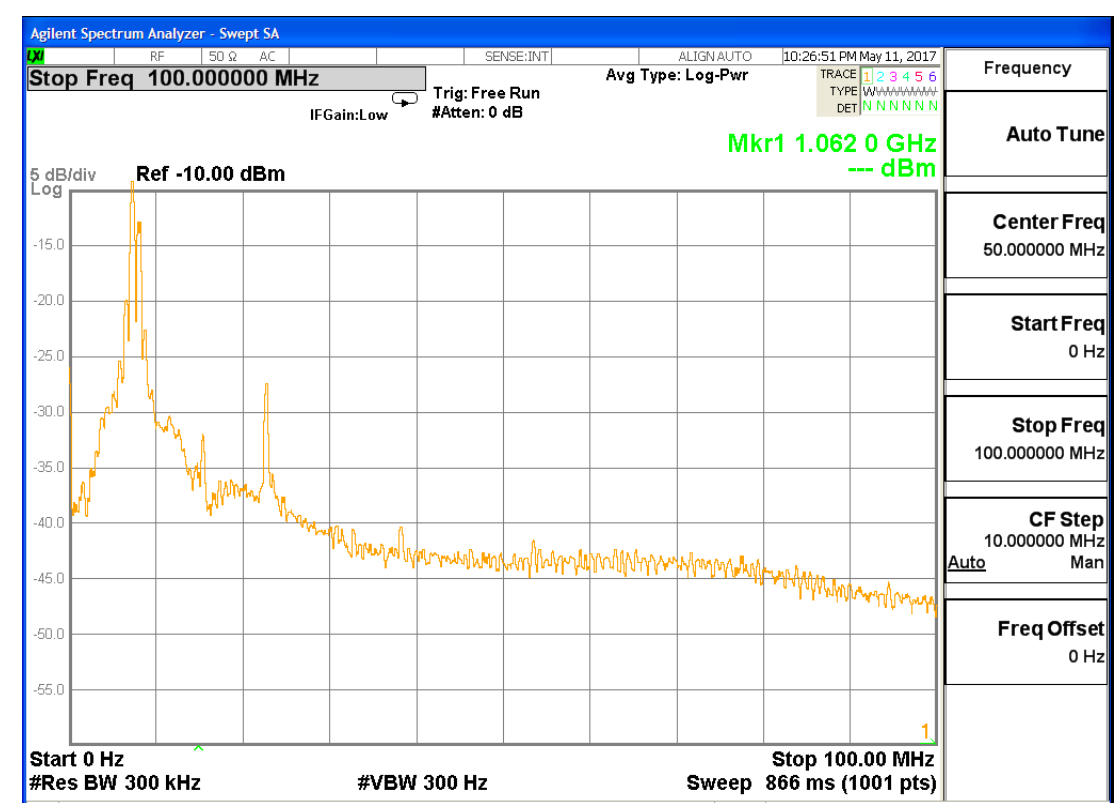

Figure 4-14 - Frequency domain output of calibration interferometer with known $100 \mathrm{pm} \Delta \lambda$.

Utilizing both Figure 4-13 and Figure 4-14, the frequency of the interference fringes $f_{i}$ is found to be $7.25 \mathrm{MHz}$. Further, with the known $100 \mathrm{pm} \Delta \lambda$, the path length 
difference that correlates with an $f_{i}$ of $7.25 \mathrm{MHz}$ can be found mathematically as follows:

$$
\begin{gathered}
\Delta \lambda=100 \mathrm{pm} \\
\Delta f=\left(2.04 \times 10^{8} \mathrm{~m} / \mathrm{s}\right) *\left[\frac{100 \mathrm{pm}}{(1545 \mathrm{~nm})^{2}}\right] \\
\Delta f=8.546 \mathrm{GHz} \\
\Delta L=\frac{2.04 \times 10^{8} \mathrm{~m} / \mathrm{s}}{(8.546 \mathrm{GHz})} \\
\Delta L=2.4 \mathrm{~cm}
\end{gathered}
$$

This allows one to correlate a $\Delta L$ of $2.4 \mathrm{~cm}$ with a $f_{i}$ of 7.25 $\mathrm{MHz}$, and also allows one to be able to find an unknown path length difference if provided a known $f_{i}$ for the sweep settings of the laser used in this chapter as follows:

$$
\begin{gathered}
\frac{7.25 \mathrm{MHz}}{2.4 \mathrm{~cm}}=\frac{f_{i}}{\Delta L} \\
\Delta L=f_{i} * \frac{2.4 \mathrm{~cm}}{7.25 \mathrm{MH} \square}
\end{gathered}
$$

\subsection{Interference Fringe Test Results and Analysis}

With a method of finding path length difference $\Delta L$ from interference fringe frequencies $f_{i}$ as found in section 4.3, the interference fringe frequencies captured in Appendix D were utilized to calculate experimental path length differences and were compared with the ruler measured path length differences. 
As seen in Appendix D, for a ruler measured $\Delta L$ of $<1$ $\mathrm{cm}$, there is a $f_{i}$ of $3.25 \mathrm{MHz}$. Thus the experimental $\Delta L$ can be found mathematically as follows according to section $4.3:$

$$
\begin{gathered}
\Delta L=f_{i} * \frac{2.4 \mathrm{~cm}}{7.25 \mathrm{MHz}} \\
\Delta L=(3.25 \mathrm{MHz}) * \frac{2.4 \mathrm{~cm}}{7.25 \mathrm{MHz}} \\
\Delta L=1.075 \mathrm{~cm}
\end{gathered}
$$

This was done for all of the ruler measured path length differences and the comparison between the ruler measured $\Delta L$ and experimental $\Delta L$ (found from $f_{i}$ ) can be seen in Table 7 .

Table 7 - Comparison of ruler measured $\Delta L$ and experimental $\Delta L$ (calculated from interference fringes frequency) for various path lengths.

\begin{tabular}{|c|c|}
\hline Ruler Measured $\Delta L$ & Experimental $\Delta L$ \\
\hline$<1 \mathrm{~cm}$ & $1.075 \mathrm{~cm}$ \\
\hline$\approx 1 \mathrm{~cm}$ & $3.145 \mathrm{~cm}$ \\
\hline$\approx 11.5 \mathrm{~cm}$ & $17.66 \mathrm{~cm}$ \\
\hline$\approx 22.5 \mathrm{~cm}$ & $36.4 \mathrm{~cm}$ \\
\hline$\approx 34 \mathrm{~cm}$ & $54.8 \mathrm{~cm}$ \\
\hline$\approx 56.5 \mathrm{~cm}$ & $87.7 \mathrm{~cm}$ \\
\hline$\approx 119 \mathrm{~cm}$ & $191.3 \mathrm{~cm}$ \\
\hline$\approx 130.5 \mathrm{~cm}$ & $209.7 \mathrm{~cm}$ \\
\hline$\approx 187.5 \mathrm{~cm}$ & $N / A$ \\
\hline
\end{tabular}


Initial observations of Table 7 between the ruler measured and experimental path length differences show a large discrepancy between them that seems to get larger as the path length difference is increased. In order to get a visual of how the ruler measured $\Delta L$ and the experimental $\Delta L$ vary, they were both plotted. For the value of $<1 \mathrm{~cm}$, a value of 0 was used for this purpose. This graph can be found in Figure 4-15.

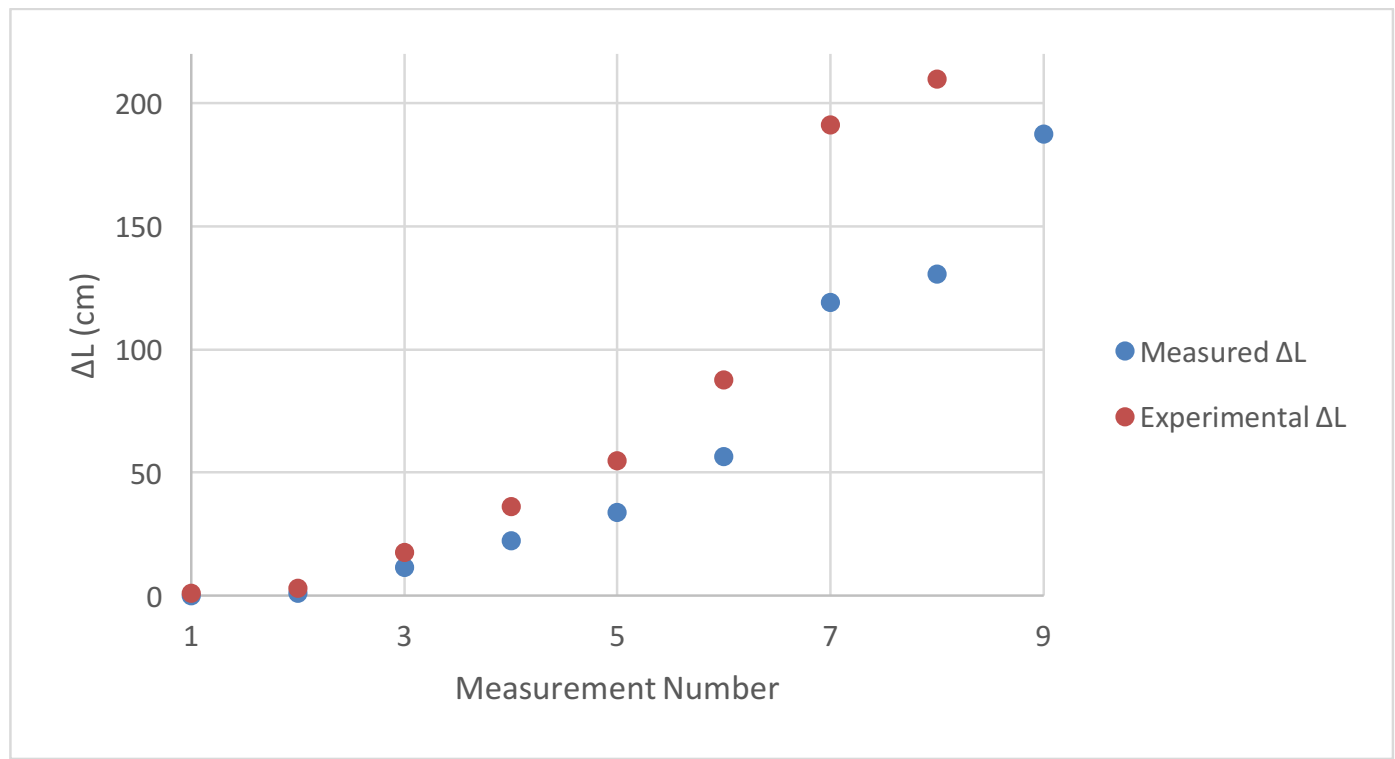

\section{Figure 4-15 - Comparison of ruler measured $\Delta L$ and} experimental $\Delta L$ for various path length measurements.

Although many more data points would have to be taken to speak with confidence, there seems to be an exponential increase in the error between the ruler measured and experimental path length differences as can be seen in the spacing between the two for each of the measurements as seen in Figure 4-15. Perhaps with more data points, the 
mathematical model can be derived for the error and can be compensated so that the experimental and ruler measured match up together closer for a more accurate ranging. Moving on to more about coherence time and coherence length, and how they hold up with the data that was found in section 4.3 , the max path length difference in which a frequency was discernible from the noise for this experiments was at a ruler measured $\Delta L$ of $130.5 \mathrm{~cm}$. With the max coherence length found to be $129.92 \mathrm{~cm}$ found in Appendix A, $130.5 \mathrm{~cm}$ is a fraction of a $\mathrm{cm}$ longer than the max coherence length found from the linewidth test in Chapter 3. Though it must be stated that although the frequency is discernible in the spectrum analyzer, it is by such a slight amount that the opposite can be argued. Not to mention the fact that the time domain interference fringes is almost completely destructive such that one would have to look at the frequency domain to obtain a frequency for it. It is fair to say that at $130.5 \mathrm{~cm}$, the coherence is being stretched. Nonetheless, there is also a possibility there may be a maximum coherence length (minimum linewidth) that was not seen in the specific wavelengths tested across the range of theoretically infinite wavelengths. The interference fringes for the path length difference of $130.5 \mathrm{~cm}$ can be seen in time domain 
with the oscilloscope in Figure 4-16 and in frequency domain with the spectrum analyzer in Figure 4-17.

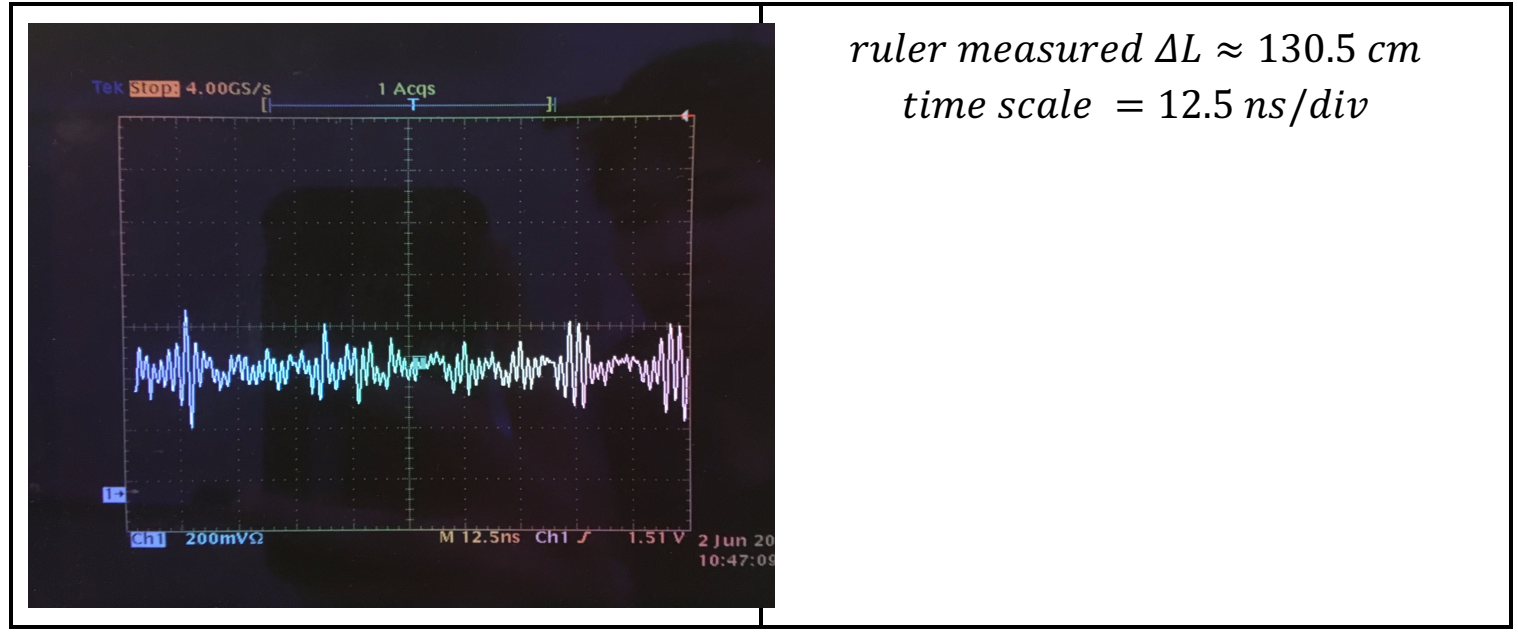

Figure 4-16 - Barely discernible interference fringes at a ruler measured path length difference of $130.5 \mathrm{~cm}$.

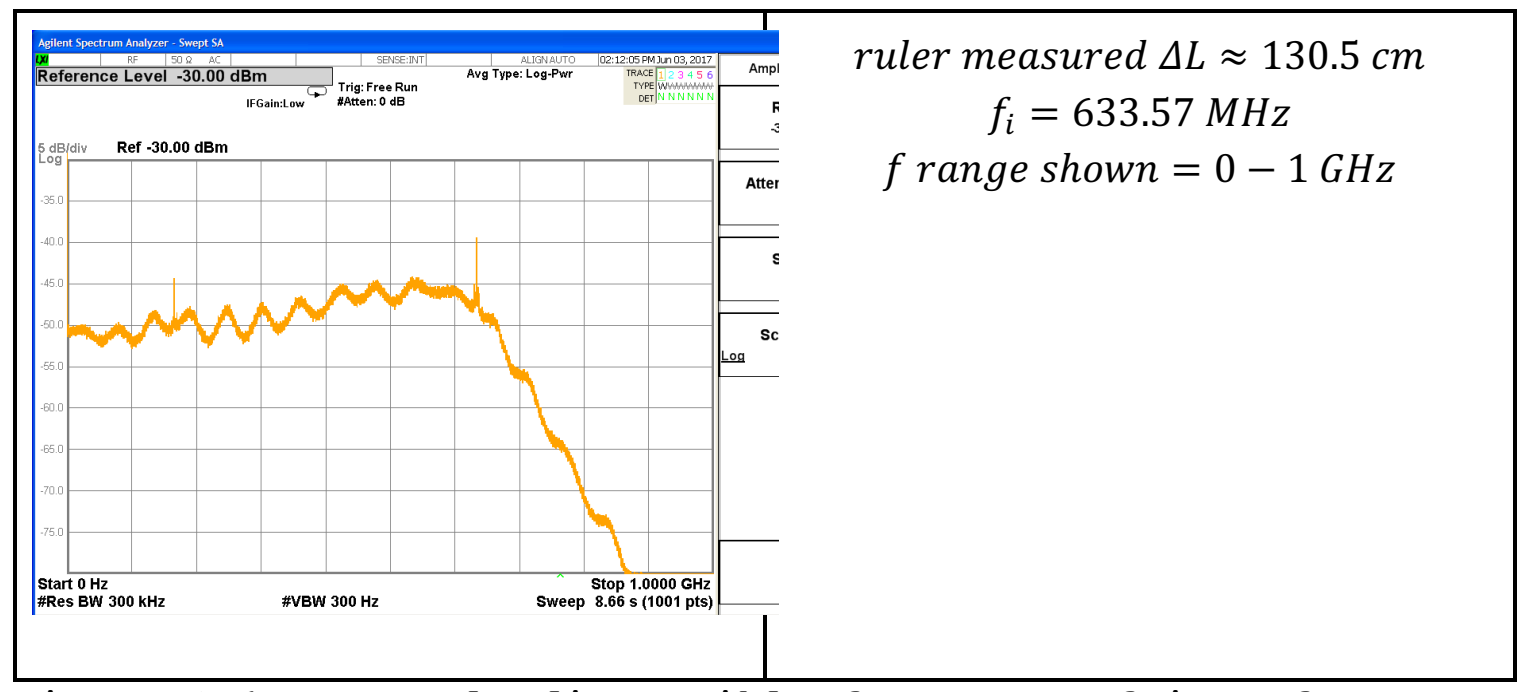

Figure 4-17 - Barely discernible frequency of interference fringes at a ruler measured path length difference of 130.5 cm.

In the larger $\Delta L$ of $187.5 \mathrm{~cm}$ that was also tested, there was neither a discernible waveform in the time domain as seen in Figure 4-18 nor a discernible frequency peak above the noise floor as seen in Figure 4-19. As such, the 
$f_{i}$ is labeled as $\mathrm{N} / \mathrm{A}$ and an experimental path length difference cannot be calculated for this case.

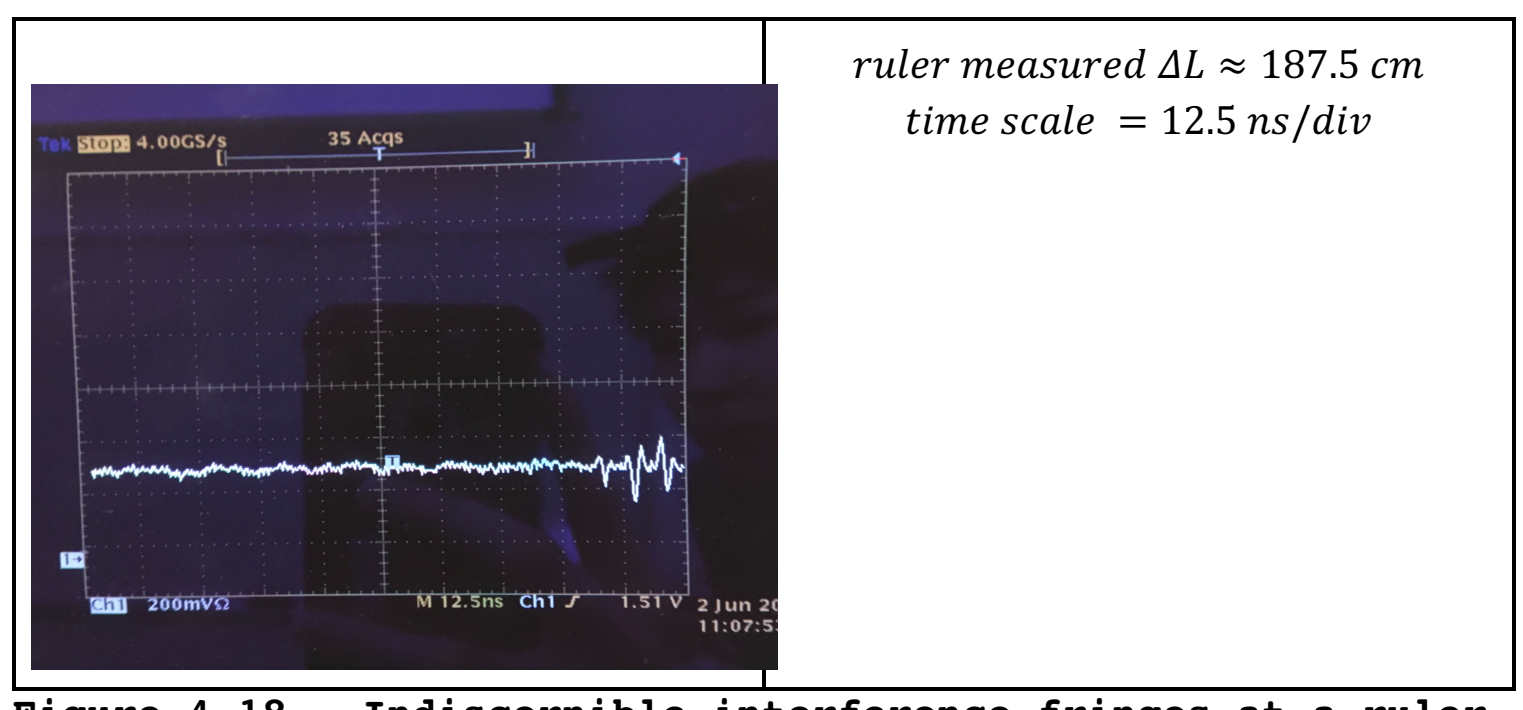

Figure 4-18 - Indiscernible interference fringes at a ruler measured path length difference of $187.5 \mathrm{~cm}$.

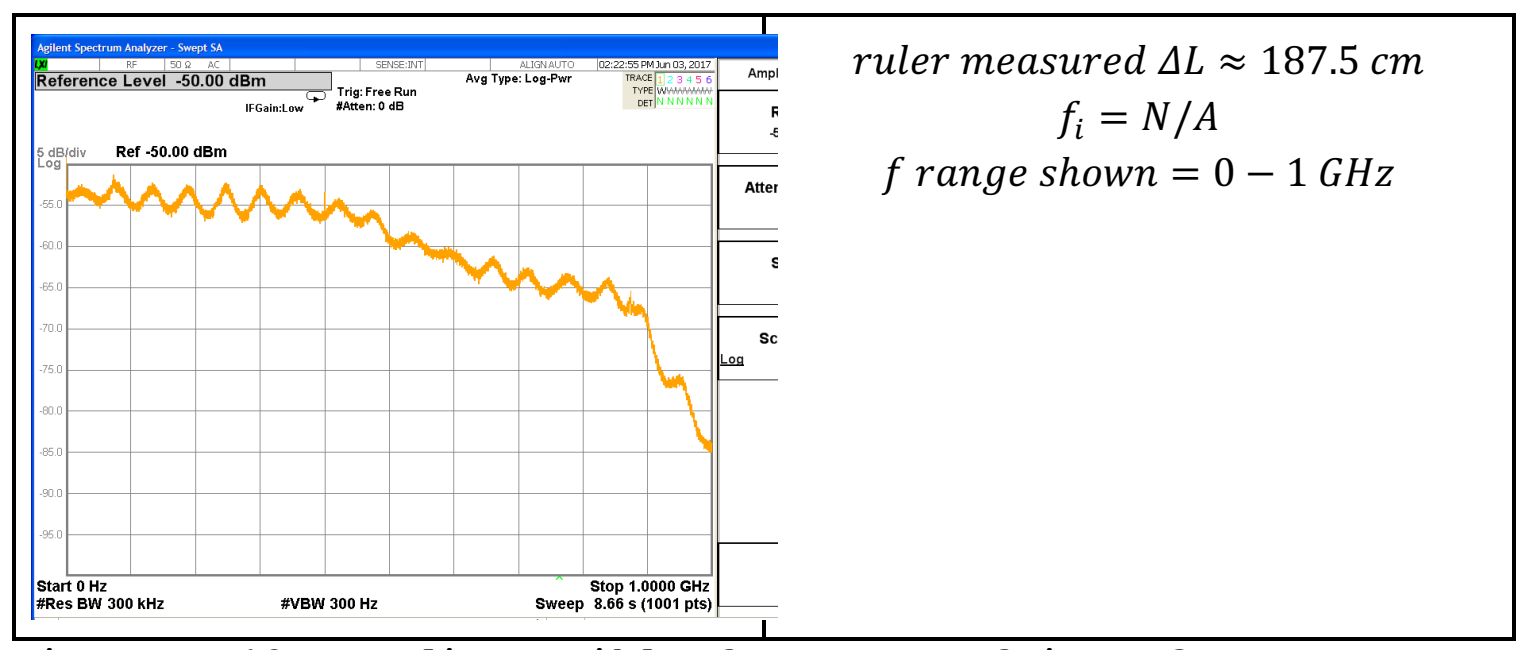

Figure 4-19 - Indiscernible frequency of interference fringes at a ruler measured path length difference of 187.5 $\mathrm{cm}$.

Given that a path length difference of $187.5 \mathrm{~cm}$ is approximately $57.5 \mathrm{~cm}$ longer than the maximum calculated coherence length of $129.92 \mathrm{~cm}$, it is understandable that there is no discernable frequency. 


\section{Chapter 5. Conclusions}

The Insight VT-DBR Akinetic laser source has been characterized for ranging performance through both a linewidth experiment and ranging experiments using interference fringes. Conclusions on the experiments, possible applications, and future work are presented. The purpose of this study was to investigate into the feasibility of using this laser for ranging purposes similar to how the FMCW method works for radar in order to combine the benefits of both a lidar and radar into one FMCW lidar sensor. The overall goals of such a sensor are outlined in Table 8 . 
Table 8 - Goals of the FMCW Lidar sensor within various parameters.

\begin{tabular}{|c|c|c|}
\hline Parameter & Goal Value & Comments \\
\hline Range/Sensitivity & $100 \mathrm{~m}$ & $\begin{array}{l}\text { Should be } \\
\text { able to sense } \\
\text { something } \\
\text { like a stop } \\
\text { sign at } 100 \mathrm{~m} \\
\text { with adequate } \\
\text { signal to } \\
\text { noise ratio. }\end{array}$ \\
\hline $\begin{array}{l}\text { Distance } \\
\text { Resolution }\end{array}$ & $10 \mathrm{~cm}$ & $\mathrm{~N} / \mathrm{A}$ \\
\hline $\begin{array}{l}\text { Time to take a } \\
\text { single data } \\
\text { point. }\end{array}$ & $2 \mu s$ & $\begin{array}{l}\text { Corresponds } \\
\text { to taking } 500 \\
\text { kSamples/s }\end{array}$ \\
\hline $\begin{array}{l}\text { Security of } \\
\text { system against } \\
\text { other lidars. }\end{array}$ & TBD & $\begin{array}{l}\text { Must be able } \\
\text { to work in an } \\
\text { environment } \\
\text { with many } \\
\text { simultaneous } \\
\text { LIDARs in } \\
\text { operation, } \\
\text { with little } \\
\text { interference. }\end{array}$ \\
\hline $\begin{array}{l}\text { Security against } \\
\text { intentional } \\
\text { jamming. }\end{array}$ & TBD & $\begin{array}{l}\text { Must still } \\
\text { work even } \\
\text { with } \\
\text { intentional } \\
\text { laser jamming } \\
\text { signal. }\end{array}$ \\
\hline Cost & TBD & $\begin{array}{l}\text { Minimal part } \\
\text { of autonomous } \\
\text { system cost }\end{array}$ \\
\hline Eye Safety & IEC Class 1 & $\begin{array}{l}\text { Class } 1 \\
\text { lasers are } \\
\text { very low risk } \\
\text { and "safe } \\
\text { under } \\
\text { reasonably } \\
\text { foreseeable } \\
\text { use" [14]. }\end{array}$ \\
\hline
\end{tabular}

\subsection{Insight Laser Linewidth}

In summary of the laser linewidth characterization performed in Chapter 3, the Insight VT-DBR akinetic laser was put through a linewidth test in which the purpose was 
to find the magnitude and variation of linewidths across its sweep range of 1522.13 to $1566.18 \mathrm{~nm}$. This was done to investigate where there may be lower linewidths across the sweep range of the laser and what causes then. In order to capture linewidths of the laser, the laser was placed into fixed wavelength mode to output across the range in integer wavelengths of $1523 \mathrm{~nm}$ to $1566 \mathrm{~nm}$ in steps of $1 \mathrm{~nm}$. The linewidth at these wavelengths were extracted using the Delayed Self-Homodyne technique using a Mach-Zehnder Interferometer. The largest linewidth captured was $300 \mathrm{MHz}$ at a fixed wavelength laser output of $1562 \mathrm{~nm}$ while the smallest linewidth was captured to be $50 \mathrm{MHz}$ found at fixed wavelength laser outputs of 1523, 1525, 1539, 1545, and $1546 \mathrm{~nm}$. With the linewidths obtained across the range of the laser, the laser pump currents at these different wavelengths were investigated in order to find a correlation between the laser currents and the linewidth. It was found that there was not a simple correlation or obvious correlation as would be hoped between the laser currents and the linewidth. However, there seems to be a loose correlation, but a correlation nonetheless, between the where one is in the wavelength range able to be output, the currents at those wavelengths, and low linewidth. Across the range of 1523 to $1566 \mathrm{~nm}$, this experiment found 
a somewhat linear, albeit low correlation, area of low linewidths depending on the front mirror and back mirror currents. It is suggested that the front mirror and back mirror currents must both be within their respective area of low linewidths for the overall laser to have a low linewidth. The front mirror currents of low linewidths vs high linewidths are shown in Figure 5-1 while the same except for back mirror currents is shown in Figure 5-2.

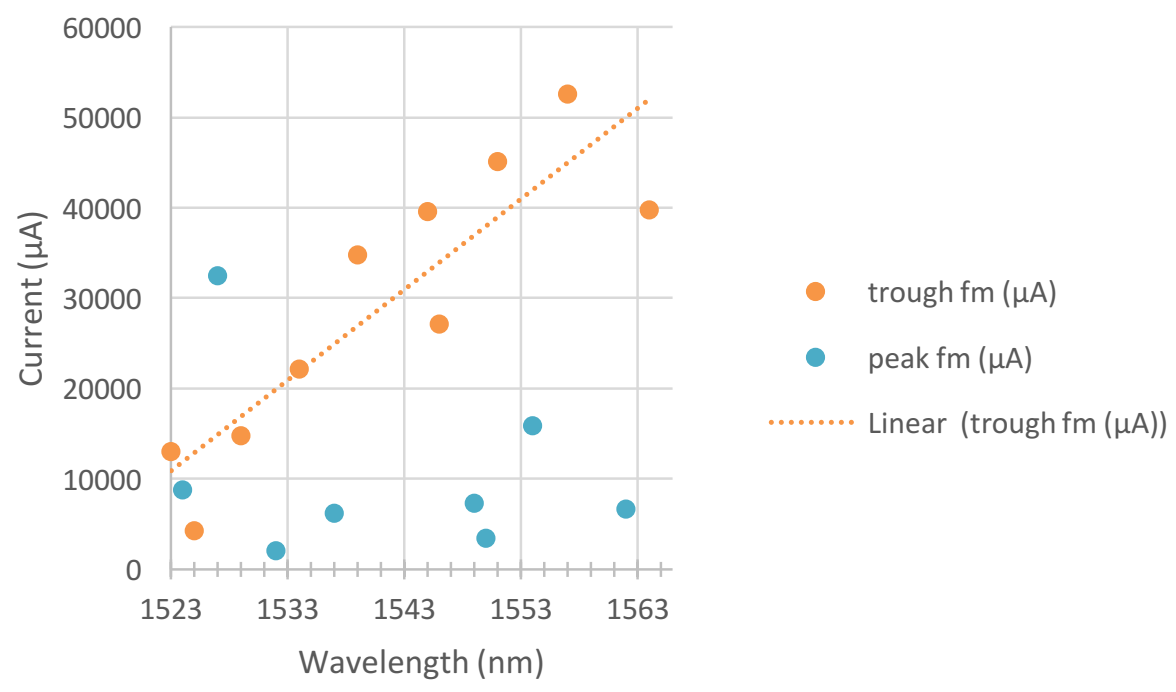

Figure 5-1 - Low linewidth front mirror (trough $\mathrm{fm}$ ) and high linewidth front mirror (peak $\mathrm{fm}$ ) currents plotted on same graph to highlight deviation of high linewidth currents from proposed trend of low linewidth currents. 


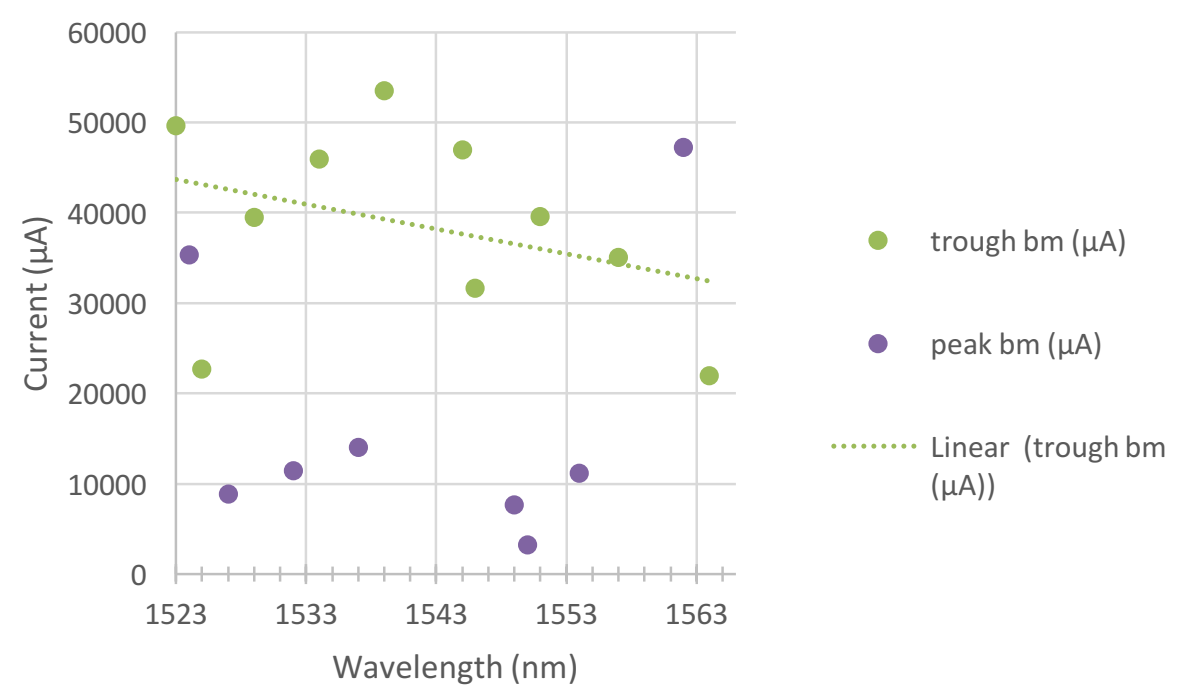

Figure 5-2 - Low linewidth back mirror (trough bm) and high linewidth back mirror (peak bm) currents plotted on same graph to highlight deviation of high linewidth currents from proposed trend of low linewidth currents.

Furthermore, as a precursor to the interference fringe experiment and to gain an understanding of the possible range of the laser in terms of ranging, coherence numbers were also calculated utilizing the linewidths captured with the minimum coherence time and coherence length of $1.061 \mathrm{~ns}$ and $21.65 \mathrm{~cm}$ respectively at the maximum linewidth found of $300 \mathrm{MHz}$, and with the maximum coherence time and coherence length of $6.366 \mathrm{~ns}$ and $129.92 \mathrm{~cm}$ respectively at the minimum linewidth found of $50 \mathrm{MHz}$. This is far from the goal range/sensitivity of $100 \mathrm{~m}$ as was outlined in Table 1 of Section 1.3 in Chapter 1. The linewidth required for 100 $m$ within optical fiber requires a much narrower linewidth of $649.6 \mathrm{kHz}$. The linewidth of the laser needs to be 
reduced by approximately 100 times to meet the $100 \mathrm{~m}$ goal outlined in Table 8 .

\subsection{Ranging with Insight Laser}

In summary of the ranging with interference fringes experiment in Chapter 4, the Insight VT-DBR akinetic laser was put through a series of ranging tests with different path length differences of an interferometer in order to obtain interference fringes from which path length can be calculated. This was done to investigate the coherence lengths as was proposed by the linewidth experiment in Chapter 3, but was also to get an initial idea of how well the laser may work in terms of ranging. In order to capture the interference fringes of varying lengths, the laser was placed into swept wavelength mode across the the range of 1522.13 to $1566.18 \mathrm{~nm}$. The interference fringes were extracted using a homemade Mach-Zehnder interferometer with interchangeable path length differences around the coherence length of the laser as was found in Chapter 4. The largest path length difference in this experiment in which a frequency of the interference fringes was discernible in the frequency spectrum was at a ruler measured path length difference of $130.5 \mathrm{~cm}$ which is very close to the max coherence length found in the linewidth experiment of $129.92 \mathrm{~cm}$. 
It was found that the interference fringes in this experiment was not very accurate in terms of larger path length differences. At a small ruler measured path length difference of < $1 \mathrm{~cm}$ (no observable measurable path length difference), the interference fringes indicated an experimental path length difference of $1.075 \mathrm{~cm}$ which is approximately $1 \mathrm{~cm}$ off from the ruler measured. However, as the ruler measured path length difference increases, the difference between the ruler measured and experimental increased as well. For example, at a ruler measured path length difference of $130.5 \mathrm{~cm}$, the interference fringes indicated an experimental path length difference of 209.7 cm. This can be observed in Table 9, along with the rest of the path length differences tested.

Table 9 - The increase in error as the ruler measured path length $\Delta L$ difference increases.

\begin{tabular}{|c|c|}
\hline Ruler Measured $\Delta L$ & Experimental $\Delta L$ \\
\hline$<1 \mathrm{~cm}$ & $1.075 \mathrm{~cm}$ \\
\hline$\approx 1 \mathrm{~cm}$ & $3.145 \mathrm{~cm}$ \\
\hline$\approx 11.5 \mathrm{~cm}$ & $17.66 \mathrm{~cm}$ \\
\hline$\approx 22.5 \mathrm{~cm}$ & $36.4 \mathrm{~cm}$ \\
\hline$\approx 34 \mathrm{~cm}$ & $54.8 \mathrm{~cm}$ \\
\hline$\approx 56.5 \mathrm{~cm}$ & $87.7 \mathrm{~cm}$ \\
\hline
\end{tabular}




\begin{tabular}{|c|c|}
\hline$\approx 119 \mathrm{~cm}$ & $191.3 \mathrm{~cm}$ \\
\hline$\approx 130.5 \mathrm{~cm}$ & $209.7 \mathrm{~cm}$ \\
\hline$\approx 187.5 \mathrm{~cm}$ & $N /$ 目 \\
\hline
\end{tabular}

A visual representation of the error between the ruler measured path lengths and experimental path lengths can be seen in Figure 5-3.

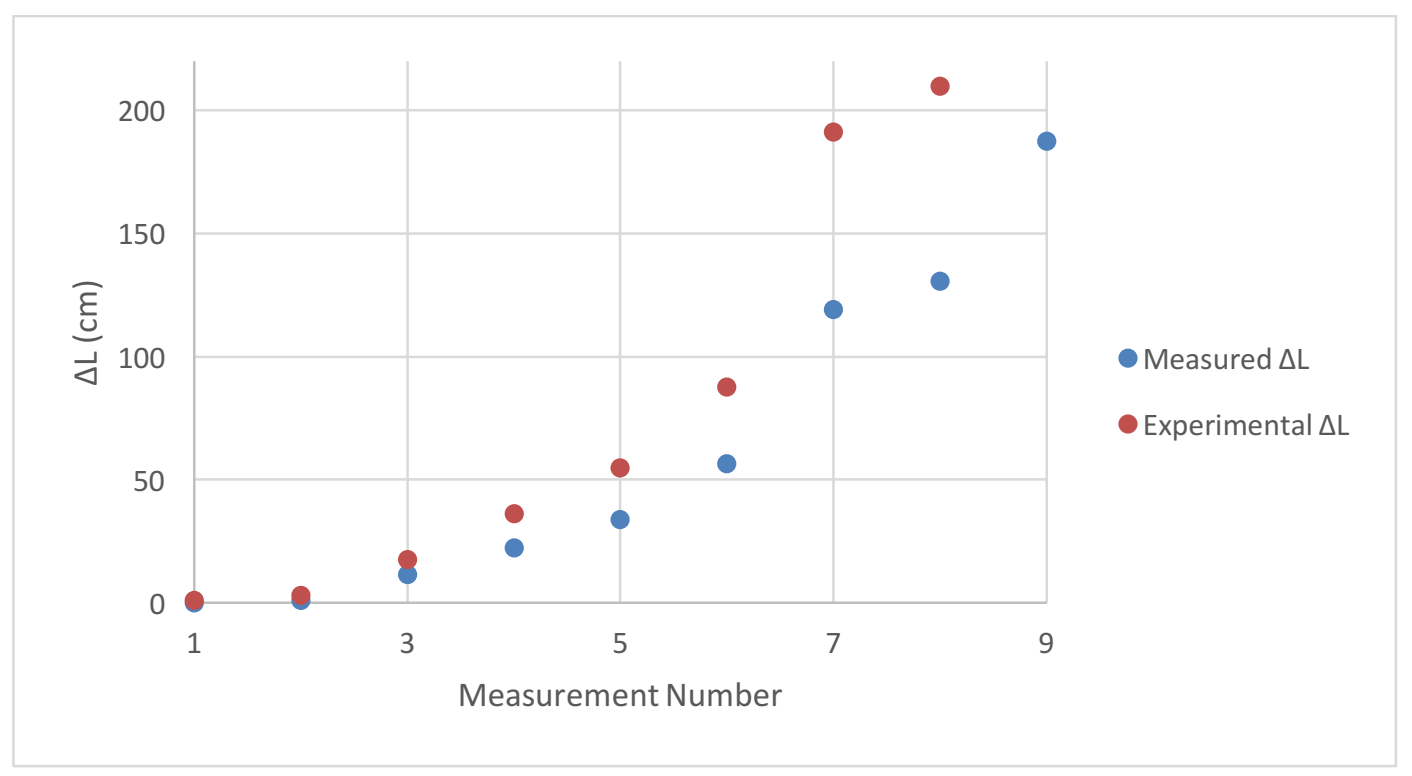

Figure 5-3 - Difference between the ruler measured path length differences and experimental path length differences as the ruler measured path length difference increases from Measurement Number 1 to 9 .

Figure 5-3 suggests that the error between the ruler measured and experimental path length differences is increasing on a sort of exponential scale. If a mathematical model for the error can be found, it can be 
used to compensate for the error found in ranging via interference fringes, making it much more accurate.

\subsection{Future Work}

This thesis was able to provide an initial investigation into the the possibility of the Insight VTDBR akinetic laser source used for ranging, and the laser shows promise. Nonetheless, there is still much investigation that needs to be done.

First, although the linewidth characterization done in Chapter 3 gives one an idea of how the linewidth varies over the laser sweep range in $1 \mathrm{~nm}$ increments, it would not be unwise to take even more data points. For example, although the linewidth experiment captured a minimum linewidth of $50 \mathrm{MHz}$, there may be another wavelength output that provides an even narrower linewidth located between the $1 \mathrm{~nm}$ increments. Theoretically, within the range of the laser of 1522.13 and 1566.18 there are an infinite number of points that can be taken. As such, in order to make the data taking simpler on the user and more efficient, one might look into the possibility of implementing an automated system, whether it be by LabVIEW or another program. This would allow one to set up the delayed selfhomodyne test and simply plug in the laser to the input of the test set up and take the output of the test setup into 
a spectrum analyzer, and step through a smaller increment of waveforms to capture many more points in between the minimum and maximum wavelengths of the laser. Taking more linewidth data points and capturing the laser currents associated with these data points would allow a user to further define the range of the currents across the sweep that lead to good linewidths as was attempted in Chapter 3 with fewer linewidths.

Further work regarding linewidth, would be to find a method by which one can minimize the linewidth of the laser. With the current linewidths of the laser, the maximum coherence length is around $130 \mathrm{~cm}$. In order for this sensor to be applicable to most systems, a range of 100 meters would be ideal. Beginning with the areas of lower linewidth to maximize effectiveness, one may want to implement a system similar to a phase-locked loop to minimize phase jumps thus allowing the linewidth to be narrower and the coherence length to be longer.

Second, although the interference fringe ranging test done in Chapter 4 was an initial investigation and was able to provide an idea of how the tunable laser would measure distance, there is much more that needs to be added on to this experiment to confidently come to the conclusion that 
this laser would be successful in real world ranging applications.

The first area that needs to be investigated for ranging is to find out why the path length difference indicated by the interference fringes is not accurate to the ruler measured path length difference and how it can be fixed. What would really help in this aspect is also to take more data points to investigate if there is a mathematical relationship of the errors between the ruler measured and experimental. An off the shelf variable length interferometer would be extremely helpful in this case due to the fact that in the interferometer made in Chapter 4 , one would have to be extremely careful with lengths due to the unpredictable lengths inherent in the process of fusion splicing, not to mention that it takes a very long time to make the variable lengths to be inserted into the interferometer of Chapter 4. If a mathematical model of the error can be found, the errors can be removed for agreement between the ruler measured and experimental path length differences.

The second area that needs to be done for ranging is for it to be done in air rather than in optical fiber as was the case in this thesis. Although for initial investigation optical fiber was used, the end goal is to 
make this ranging possible in the open. This would bring in a variety of different factors into play such as scattering

The third area to be investigated is how well the laser is able to perform ranging when the object is moving. In this thesis, the experiments were done with a constant path length difference, but it is necessary to investigate how the laser performs with a changing path length difference and see if it can detect the rate of change. This would be followed by an in the open test (not in optical fiber) with a moving object and analysis of if the laser can not only detect the object and how far it is away, but also how fast the object is moving. This would being in a variety of different factors into play such as the Doppler Effect. 


\section{BIBLIOGRAPHY}

i[1] "Journey - Waymo." Waymo. N.p., n.d. Web. 14 Oct. 2017. aaa <https://waymo.com/journey/>.

[2] Alex Davies, "We Take a Ride in the Self-Driving Uber Now Roaming Pittsburgh." Wired. Conde Nast, 14 Sept. 2016. Web. 4 Jan. 2017. <https://www.wired.com/2016/09/selfdriving-autonomous-uber-pittsburgh/>.

[3] Fred Lambert, "Tesla Announces All Production Cars Now Have Fully Self-driving Hardware." Electrek. N.p., 19 Oct. 2016. Web. 7 Jan. 2017. <https://electrek.co/2016/10/19/tesla-fully-autonomousself-driving-car/>.

[4] Alex Davies, "Turns Out the Hardware in Self-Driving Cars Is Pretty Cheap." Wired. Conde Nast, 10 May 2017. Web. 11 May 2017. <https://www.wired.com/2015/04/cost-of-sensorsautonomous-cars $/>$.

[5] "Centimeter-accurate GPS for Self-driving Vehicles." Articles. N.p., n.d. Web. 24 Jan. 2017. <http://articles.sae.org/15067/>.

[6] "What Is an Ultrasonic Sensor?" Ultrasonic Sensor / What Is an Ultrasonic Sensor? N.p., n.d. Web. 23 Jan. 2017. <http://education.rec.ri.cmu.edu/content/electronics/boe/u ltrasonic_sensor/1.html>.

[7] "Odometry Sensors." Title3.gif. N.p., n.d. Web. 23 Jan. 2017. <http://planning.cs.uiuc.edu/node576.html>.

[8] United States Geological Survey, N.p., n.d. Web. 13 May 2017. <https://lta.cr.usgs.gov/lidar_digitalelevation>.

[9] "Autonomous Cars' Pick: Camera, Radar, Lidar? | EE Times." EETimes. N.p., n.d. Web. 30 Jan. 2017.

$<$ http: //www.eetimes.com/author.asp? section_id=36\&doc_id=13 $30069>$.

[10] Christian Wolff, Radar Basics. N.p., n.d. Web. 2 Feb. 2017.

<http: / / www . radartutorial .eu/02 basics/Frequency\%20Modulat ed\%20Continuous $\% 2$ Wave 20 Radar.en.html> .

[11] Tobiasotto, "Principle of FMCW Radar." LinkedIn Slideshare. N.p., 26 July 2012. Web. 14 Feb. 2017. <https://www.slideshare.net/tobiasotto/principle-of-fmcwradars $>$. 
[12] The Universe, N.p., n.d. Web. 11 May 2017.

<http://wps.pearsoned.com.au/sf3_2/130/33327/8531808.cw//8531815/index.html>.

[13] Christian Martens, "Sweep Stability Characterization of a Vernier-Tuned Distributed Bragg Reflector (VT-DBR) AllSemiconductor Tunable Swept Laser System at $1550 \mathrm{~nm}$ for Sensing Applications." California Polytechnic State University, San Luis Obispo, Thesis 2015.

[14] Laser Standards and Classifications. N.p., n.d. Web. 01 June 2017. <https://www.rli.com/resources/articles/classification.asp $\mathrm{x}>$.

[15] Jacob Egorov, "Wavelength accuracy study for high-density Fiber Bragg grating sensor systems using a Rapidly-Swept Akinetic-Laser source." California Polytechnic State University, San Luis Obispo, Thesis 2016.

[16] Gregory Bergdoll, "Characterization of two Vernier-Tuned Distributed Bragg Reflector (VT-DBR) Lasers used in swept Source Optical Coherence Tomography (SS-OCT)." California Polytechnic State University, San Luis Obispo, Thesis 2015 .

[17] Dennis Derickson et al., "SGDBR single-chip wavelength tunable lasers for swept source OCT." in SPIE Digital Library, San Jose, 2008.

[18] M. Bonesi et al., "Akinetic all-semiconductor programmable swept-source at $1550 \mathrm{~nm}$ and $1310 \mathrm{~nm}$ with centimeters coherence length." Optics Express, vol. 22, no. 3, pp. 2632-2655, 2014 .

[19] M.P. Minneman, J. Ensher, M. Crawford, and D. Derickson, "All-semiconductor high-speed akinetic swept-source for OCT." in Proc. SPIE 8311, Optical Sensors and Biophotonics III, 831116,2011 .

[20] Insight Photonics, "Atlas 3.7.0 User Manual".

[21] Dennis Derickson, "Fiber Optic Test and Measurement." Upper Saddle River Prentice-Hall, 1998. Print.

[22] Insight Photonics, [Factory Data].

[23] "Spectral Line Shape." Wikipedia. Wikimedia Foundation, 16 Apr. 2017. Web. 01 June 2017.

<https://en.wikipedia.org/wiki/Spectral_line_shape>. 


\section{APPENDICES}

\section{Appendix A. Captured Laser Linewidths}

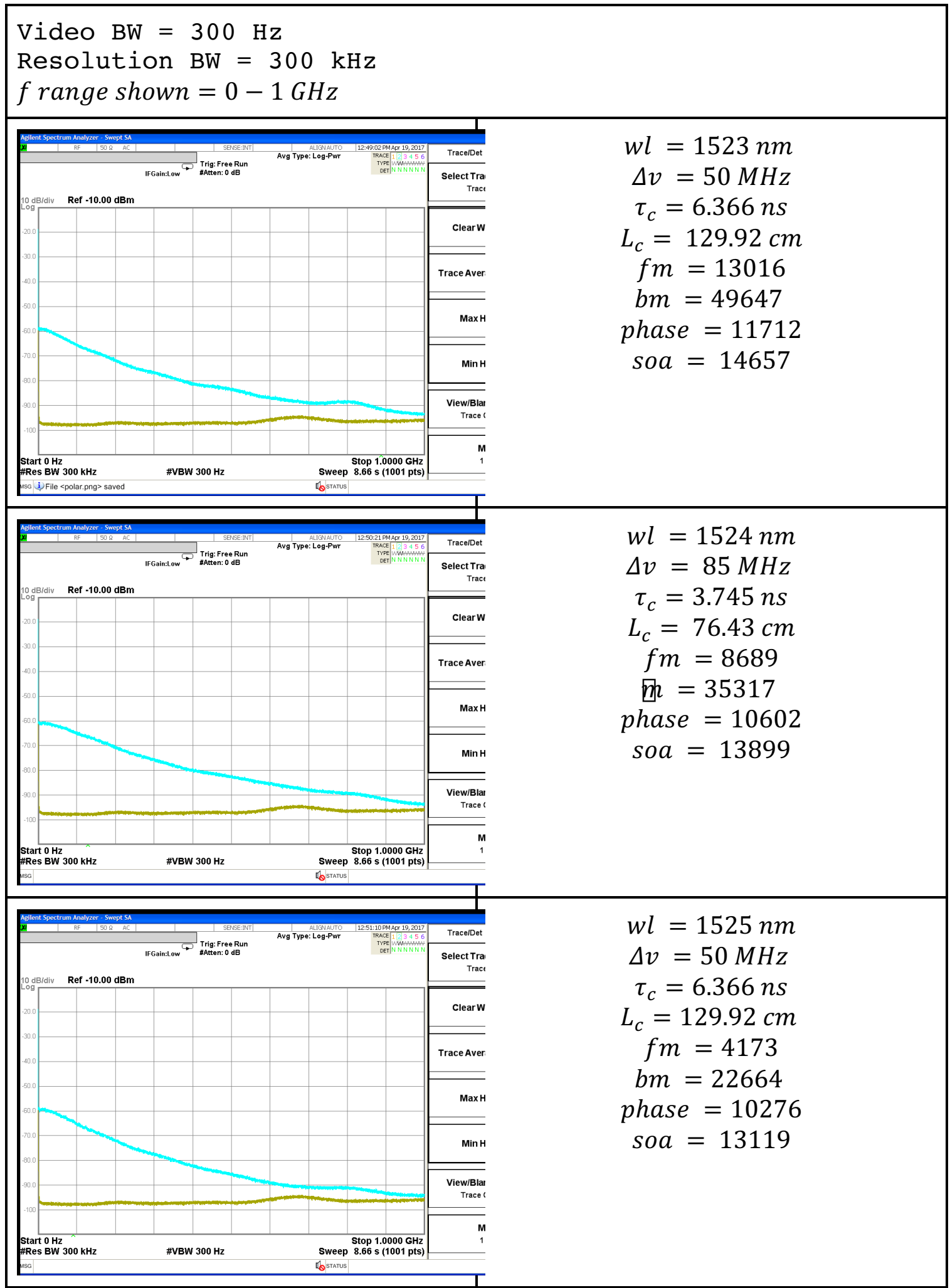




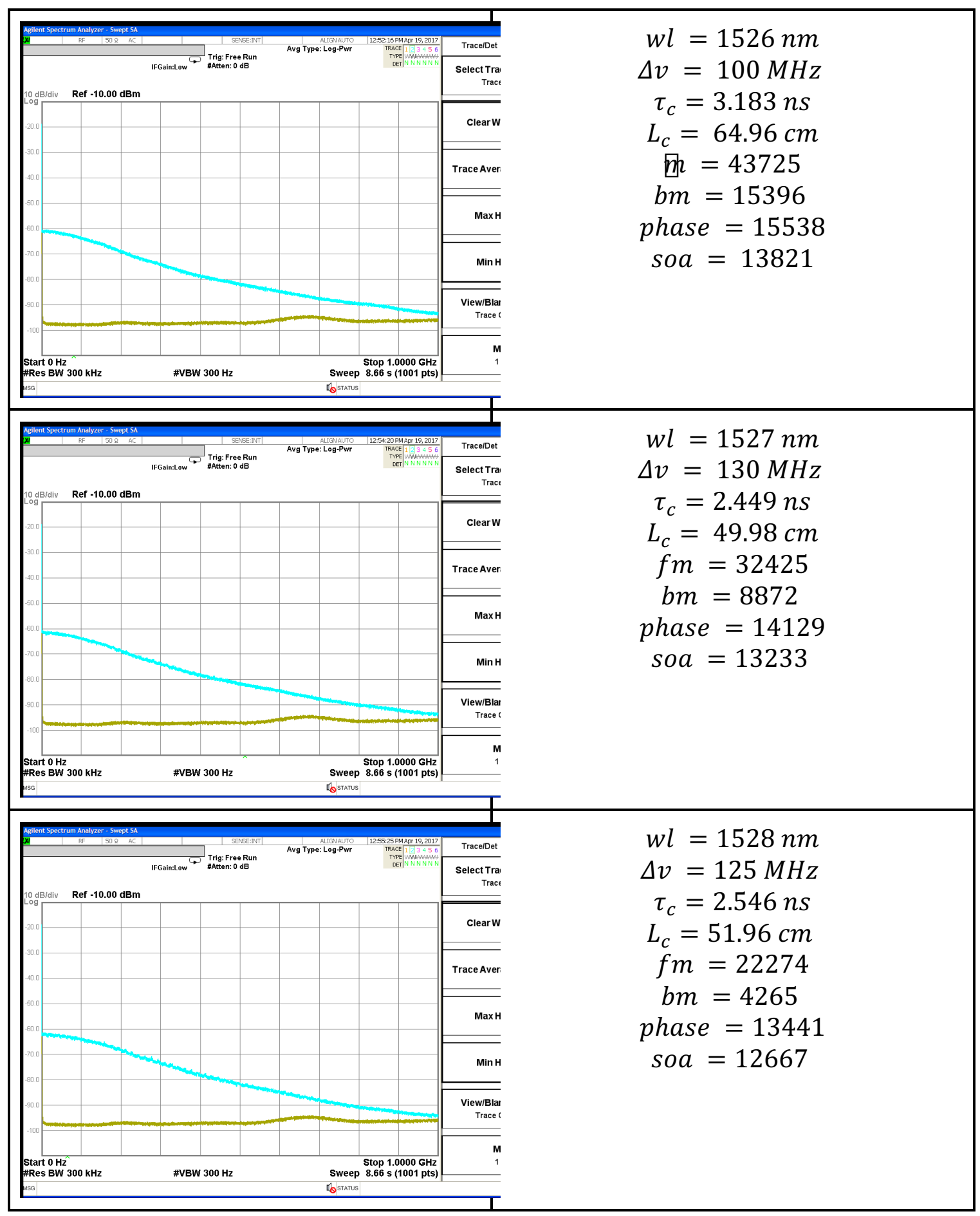




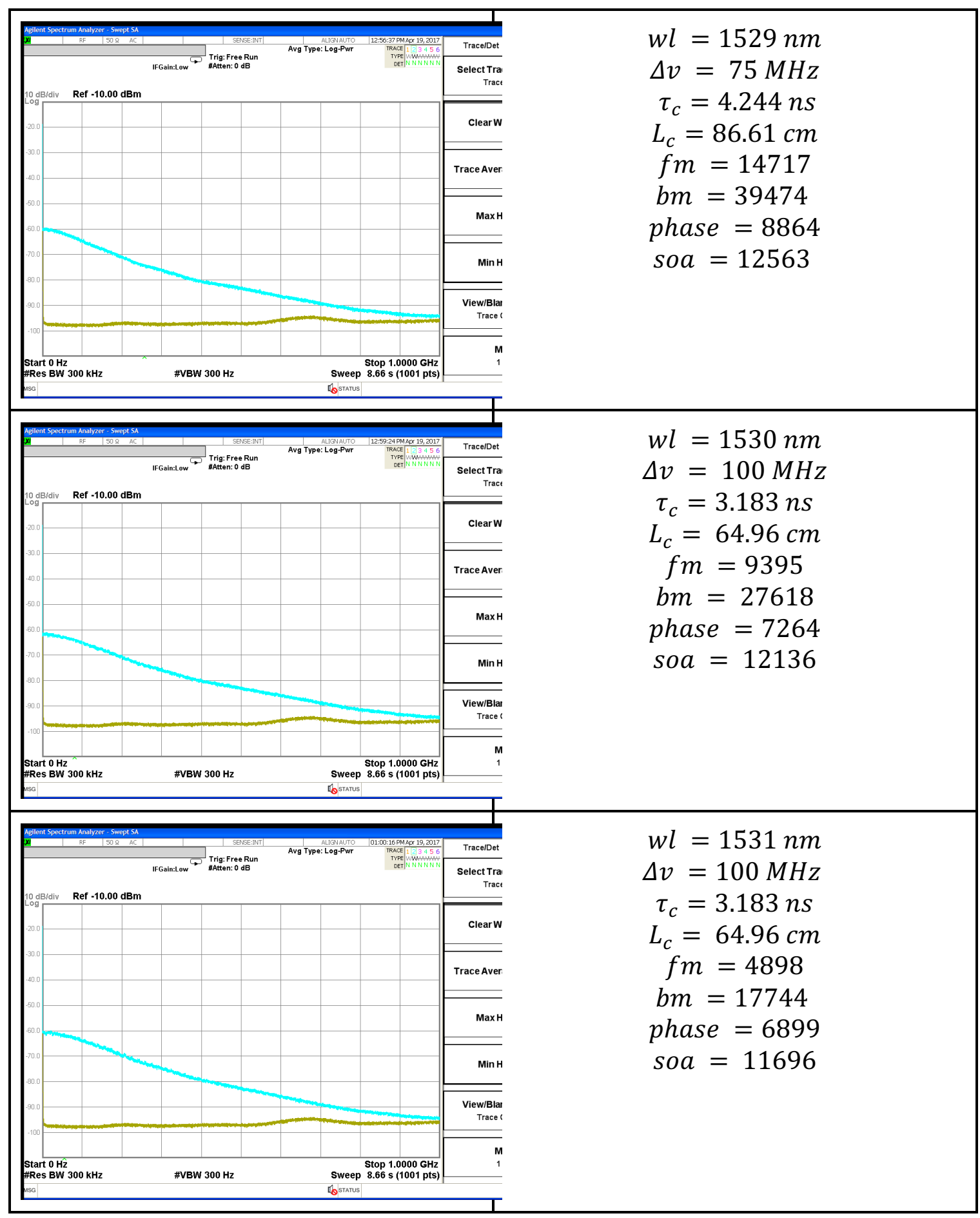




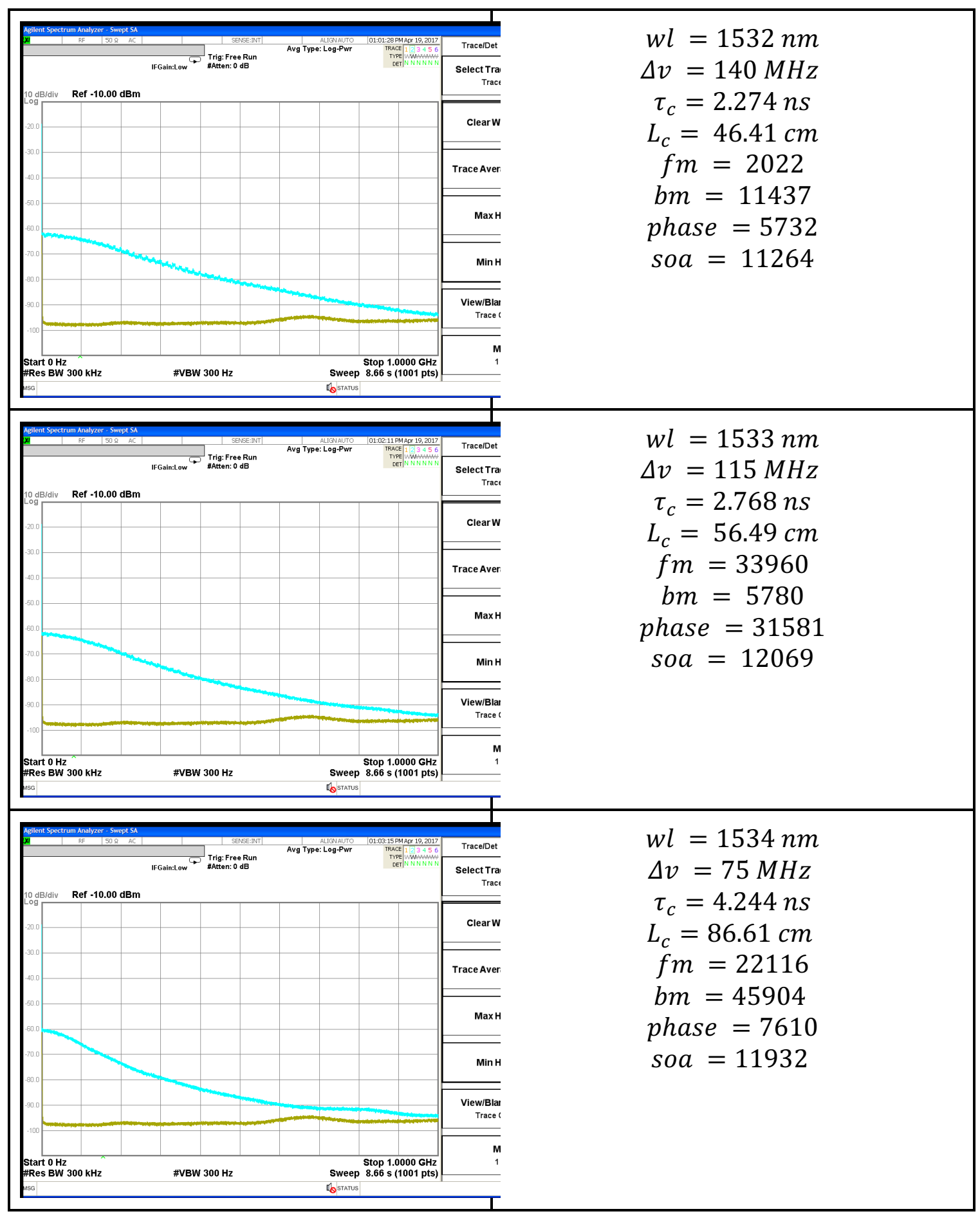




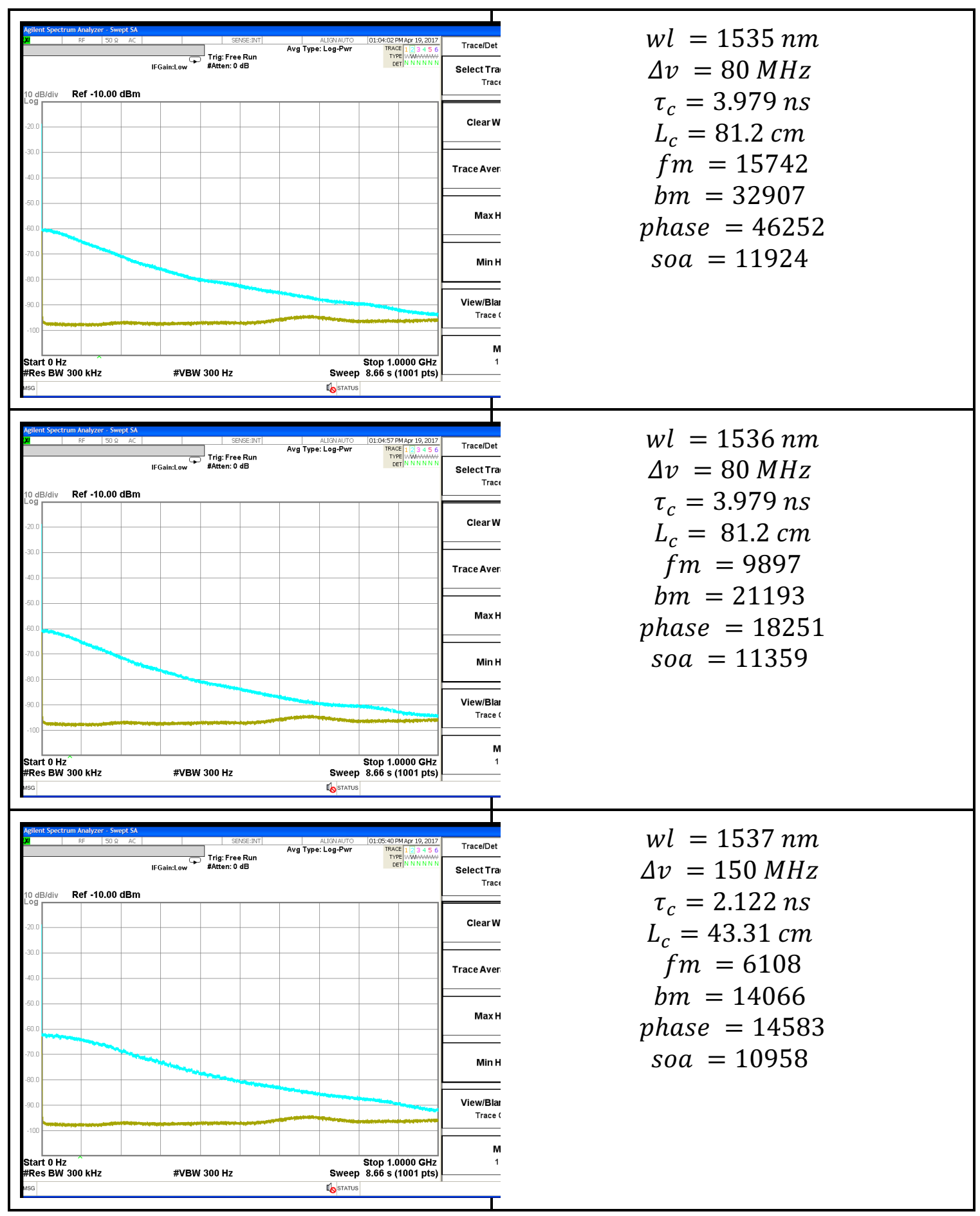




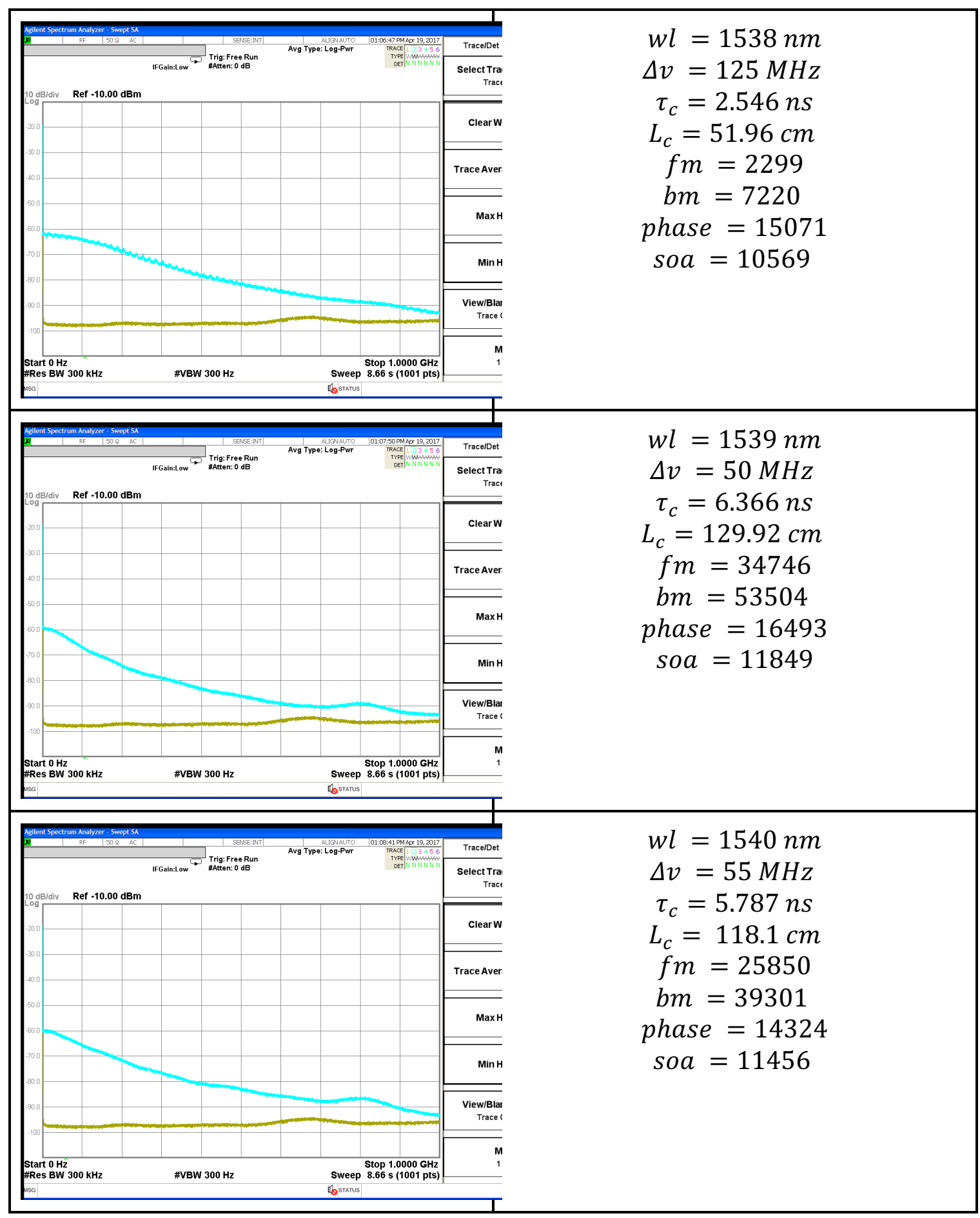



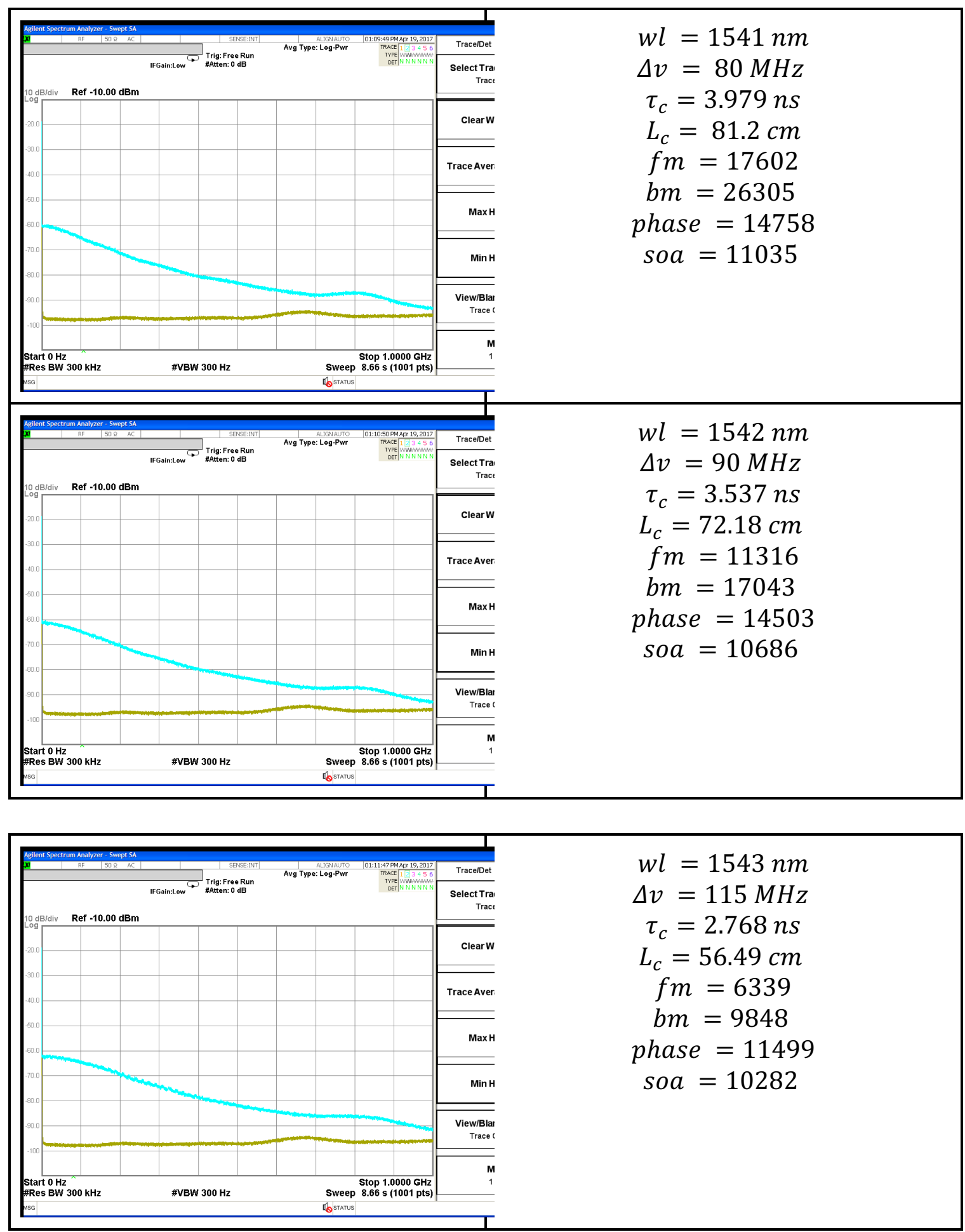

$$
\begin{gathered}
w l=1543 \mathrm{~nm} \\
\Delta v=115 \mathrm{MHz} \\
\tau_{c}=2.768 \mathrm{~ns} \\
L_{c}=56.49 \mathrm{~cm} \\
\mathrm{fm}=6339 \\
b m=9848 \\
\text { phase }=11499 \\
\text { soa }=10282
\end{gathered}
$$




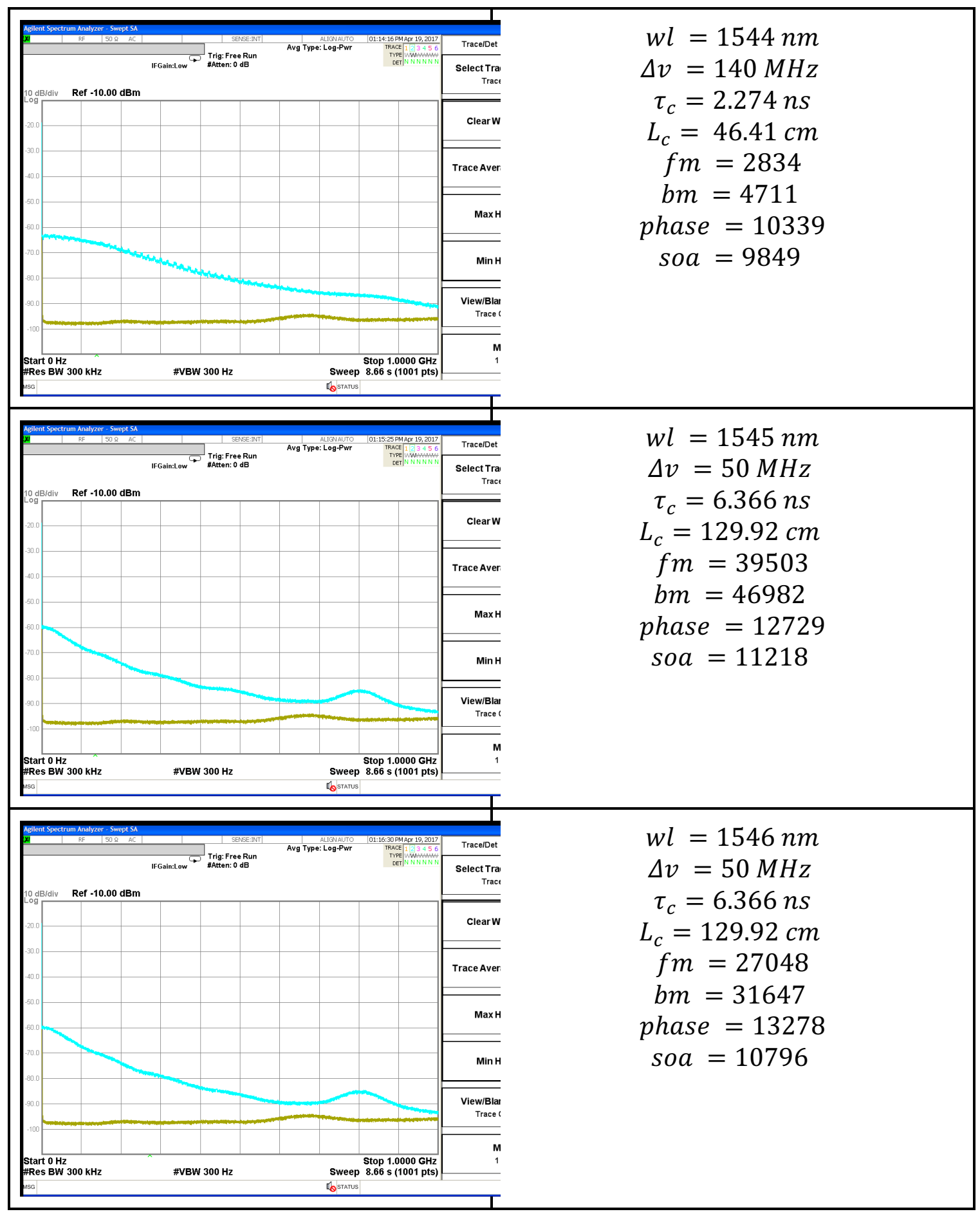




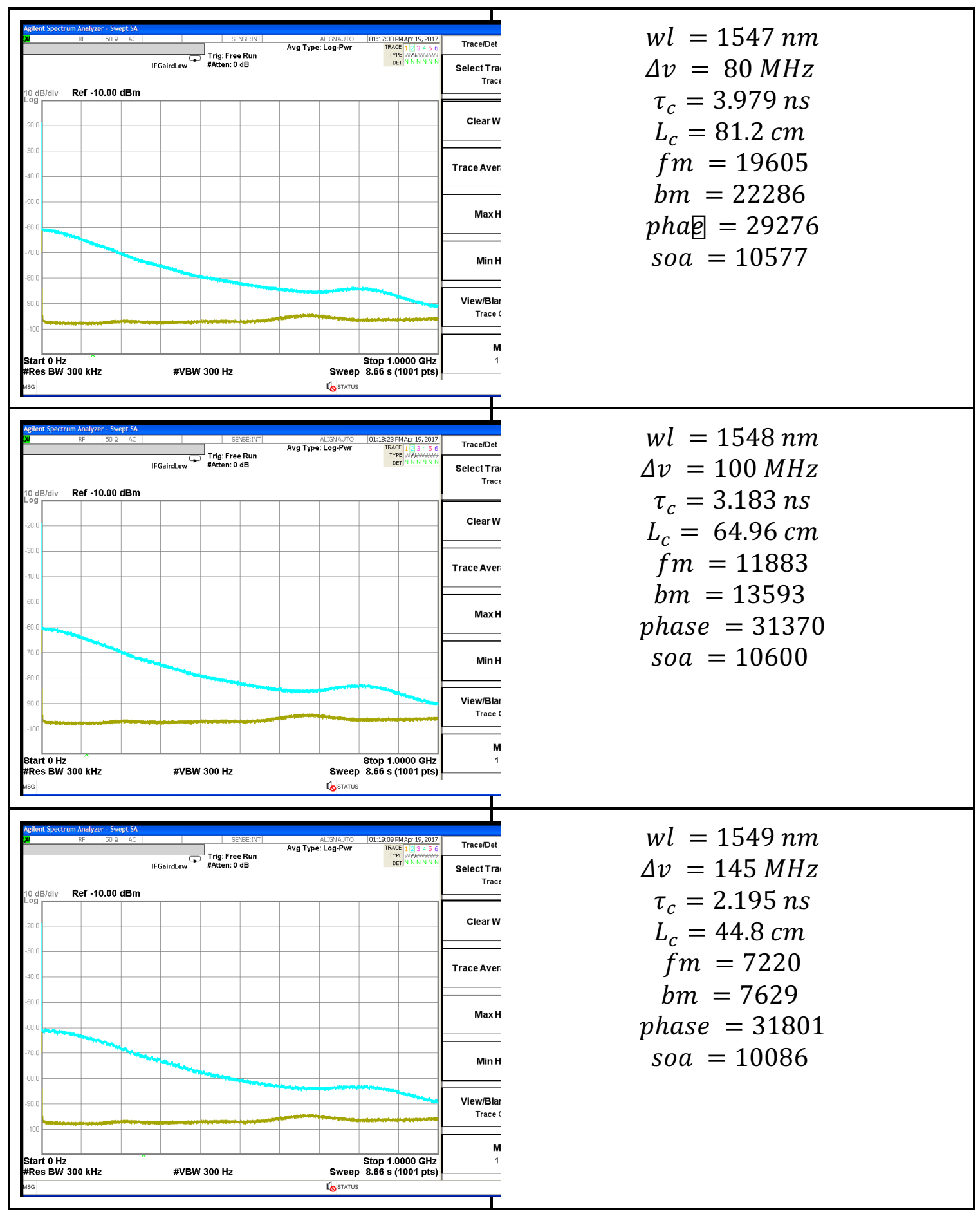




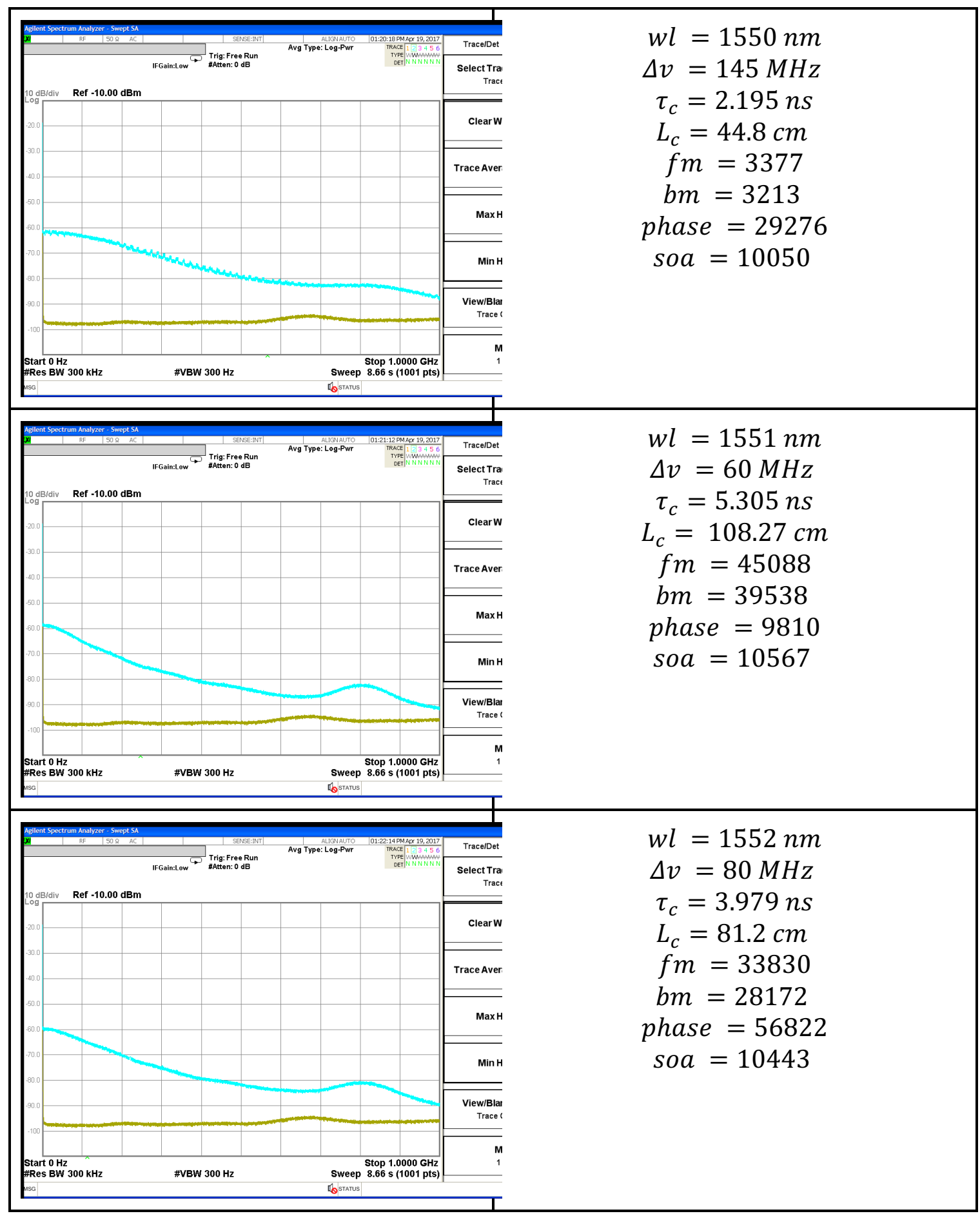




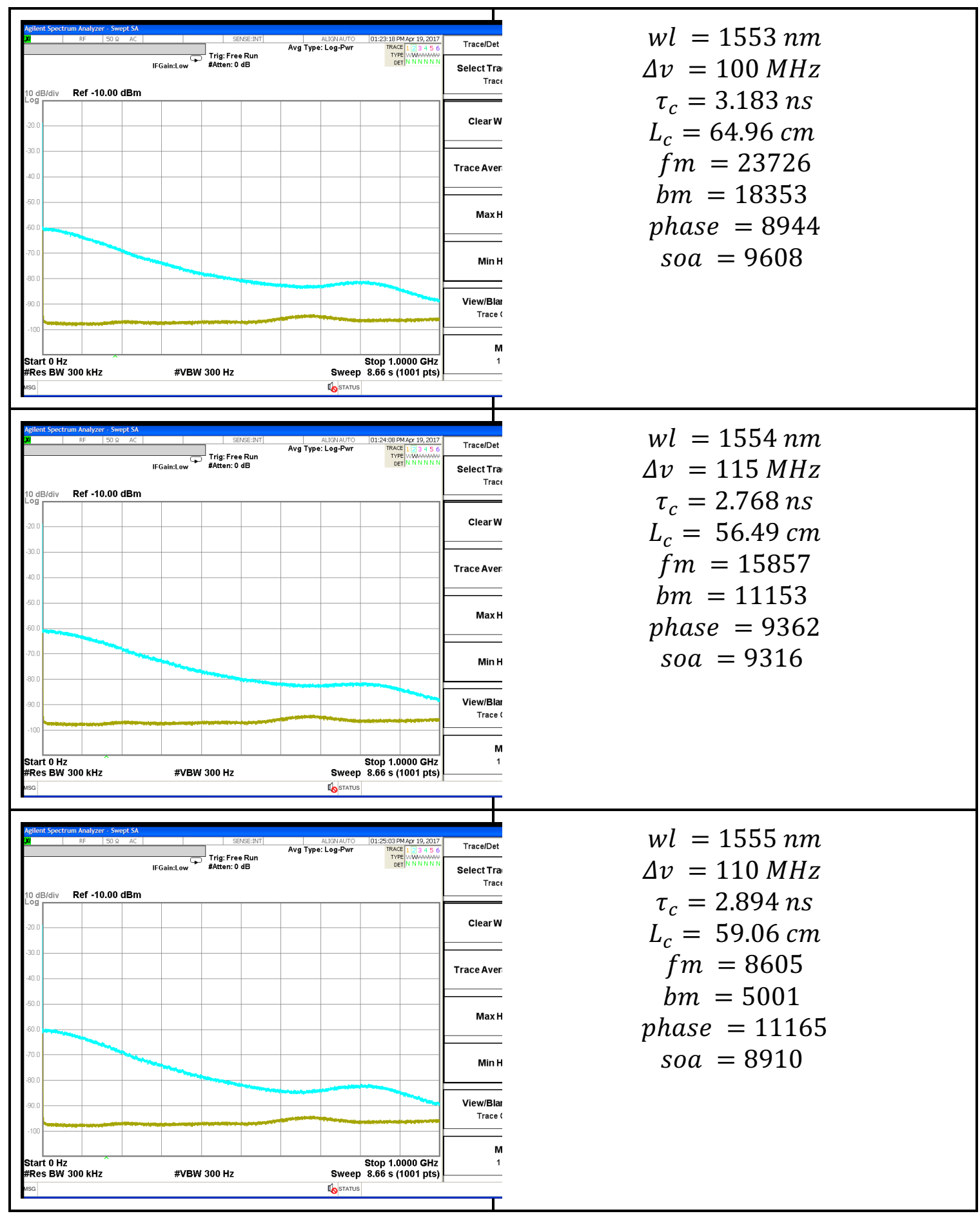




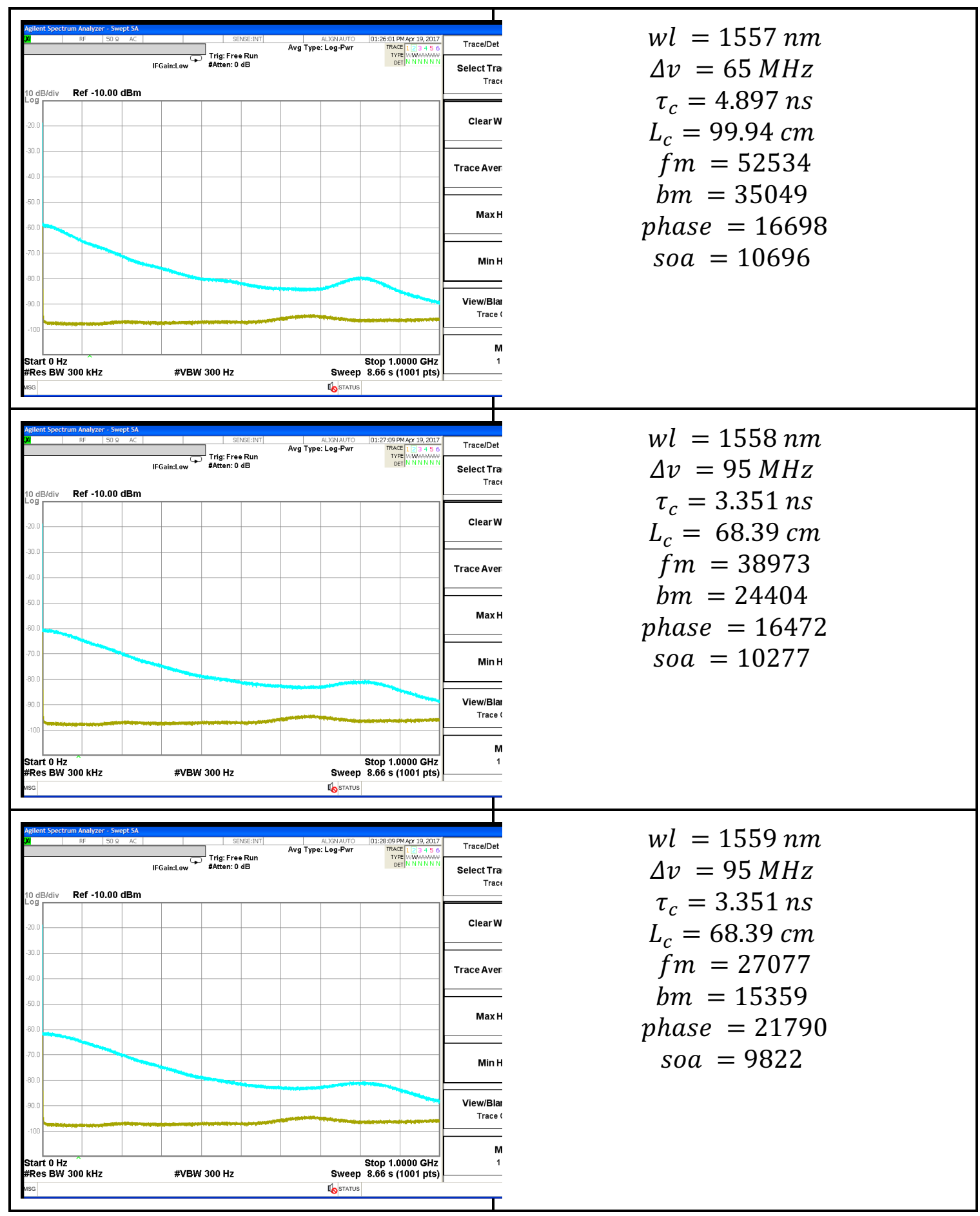




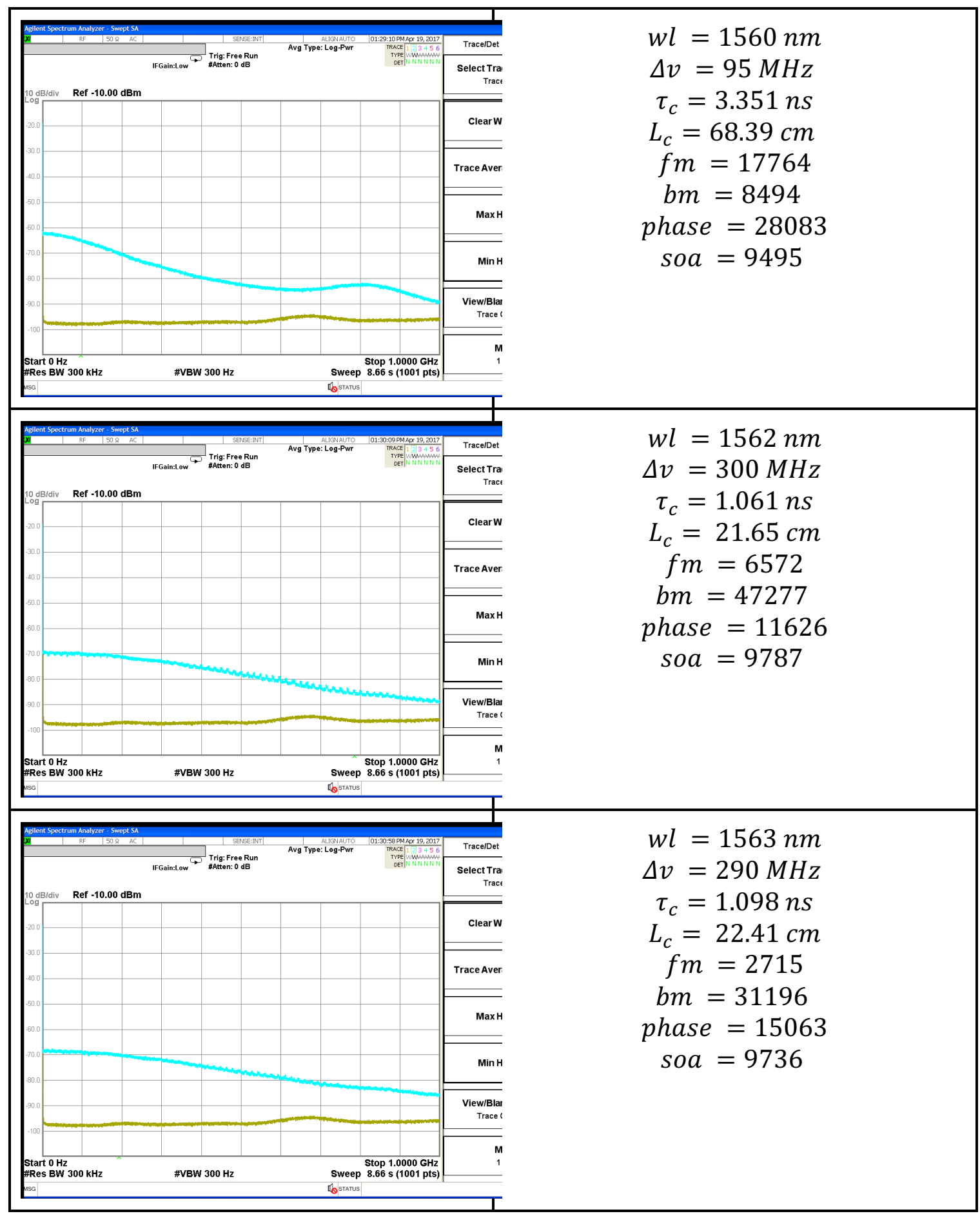



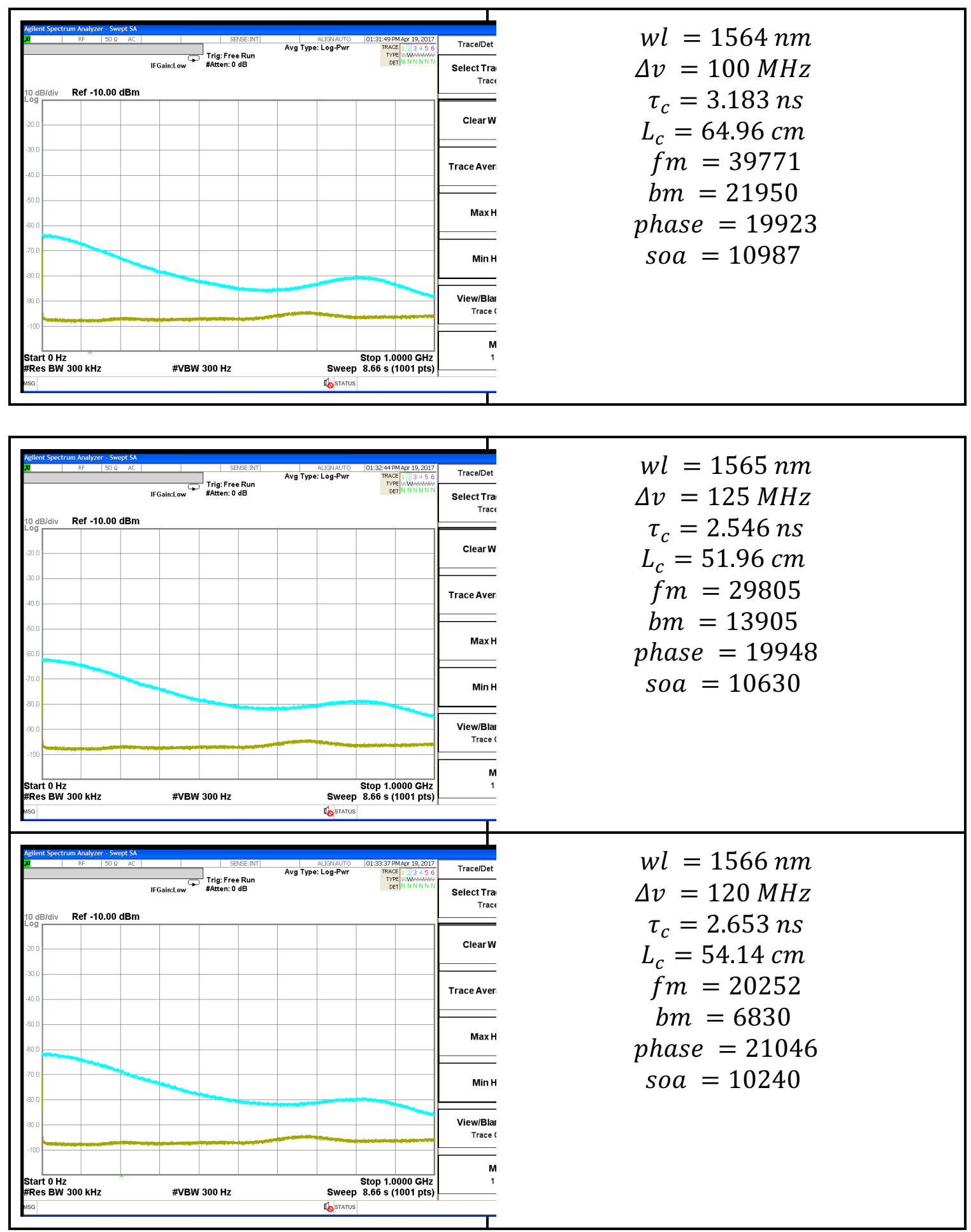
Appendix B. Laser Lineshapes

\begin{tabular}{|c|c|c|}
\hline $\begin{array}{l}\text { Laser } \\
\text { Wavelength }\end{array}$ & $\begin{array}{l}\text { Experimental Laser } \\
\text { Lineshape }\end{array}$ & $\begin{array}{l}\text { Lorentzian Laser } \\
\text { Lineshape }\end{array}$ \\
\hline $1523 \mathrm{~nm}$ & $\begin{array}{c}\Delta v=50 \mathrm{MHz} \\
\Delta v_{10 \mathrm{~dB}}=160 \mathrm{MHz} \\
\Delta v_{20 \mathrm{~dB}}=350 \mathrm{MHz} \\
\Delta v_{30 \mathrm{~dB}}=700 \mathrm{MHz}\end{array}$ & $\begin{array}{c}\Delta v=50 \mathrm{MHz} \\
\Delta v_{10 \mathrm{~dB}}=150 \mathrm{MHz} \\
\Delta v_{20 \mathrm{~dB}}=497.5 \mathrm{MHz} \\
\Delta v_{30 \mathrm{~dB}}=1580 \mathrm{MHz}\end{array}$ \\
\hline $1524 \mathrm{~nm}$ & $\begin{array}{c}\Delta v=85 \mathrm{MHz} \\
\Delta v_{10 \mathrm{~dB}}=200 \mathrm{MHz} \\
\Delta v_{20 \mathrm{~dB}}=400 \mathrm{MHz} \\
\Delta v_{30 \mathrm{~dB}}=850 \mathrm{MHz}\end{array}$ & $\begin{array}{c}\Delta v=85 \mathrm{MHz} \\
\Delta v_{10 \mathrm{~dB}}=255 \mathrm{MHz} \\
\Delta v_{20 \mathrm{~dB}}=845.7 \mathrm{MHz} \\
\Delta v_{30 \mathrm{~dB}}=2687 \mathrm{MHz}\end{array}$ \\
\hline $1525 \mathrm{~nm}$ & $\begin{array}{c}\Delta v=50 \mathrm{MHz} \\
\Delta v_{10 \mathrm{~dB}}=169 \mathrm{MHz} \\
\Delta v_{20 \mathrm{~dB}}=335 \mathrm{MHz} \\
\Delta v_{30 \mathrm{~dB}}=600 \mathrm{MHz}\end{array}$ & $\begin{array}{c}\Delta v=50 \mathrm{MHz} \\
\Delta v_{10 \mathrm{~dB}}=150 \mathrm{MHz} \\
\Delta v_{20 \mathrm{~dB}}=497.5 \mathrm{MHz} \\
\Delta v_{30 \mathrm{~dB}}=1580 \mathrm{MHz}\end{array}$ \\
\hline $1526 \mathrm{~nm}$ & $\begin{array}{c}\Delta v=100 \mathrm{MHz} \\
\Delta v_{10 \mathrm{~dB}}=230 \mathrm{MHz} \\
\Delta v_{20 \mathrm{~dB}}=470 \mathrm{MHz} \\
\Delta v_{30 \mathrm{~dB}}=875 \mathrm{MHz}\end{array}$ & $\begin{array}{c}\Delta v=100 \mathrm{MHz} \\
\Delta v_{10 \mathrm{~dB}}=300 \mathrm{MHz} \\
\Delta v_{20 \mathrm{~dB}}=995 \mathrm{MHz} \\
\Delta v_{30 \mathrm{~dB}}=3161 \mathrm{MHz}\end{array}$ \\
\hline $1527 \mathrm{~nm}$ & $\begin{array}{c}\Delta v=130 \mathrm{MHz} \\
\Delta v_{10 d B}=250 \mathrm{MHz} \\
\Delta v_{20 d B}=490 \mathrm{MHz} \\
\Delta v_{30 d B}=870 \mathrm{MHz}\end{array}$ & $\begin{array}{c}\Delta v=130 \mathrm{MHz} \\
\Delta v_{10 \mathrm{~dB}}=390 \mathrm{MHz} \\
\Delta v_{20 \mathrm{~dB}}=1293.5 \mathrm{MHz} \\
\Delta v_{30 \mathrm{~dB}}=4109 \mathrm{MHz}\end{array}$ \\
\hline $1528 \mathrm{~nm}$ & $\begin{array}{c}\Delta v=125 \mathrm{MHz} \\
\Delta v_{10 \mathrm{~dB}}=250 \mathrm{MHz} \\
\Delta v_{20 d B}=500 \mathrm{MHz} \\
\Delta v_{30 \mathrm{~dB}}=850 \mathrm{MHz}\end{array}$ & $\begin{array}{c}\Delta v=125 \mathrm{MHz} \\
\Delta v_{10 \mathrm{~dB}}=375 \mathrm{MHz} \\
\Delta v_{20 \mathrm{~dB}}=1243.7 \mathrm{MHz} \\
\Delta v_{30 \mathrm{~dB}}=3951 \mathrm{MHz}\end{array}$ \\
\hline $1529 \mathrm{~nm}$ & $\begin{array}{c}\Delta v=75 \mathrm{MHz} \\
\Delta v_{10 d B}=185 \mathrm{MHz} \\
\Delta v_{20 d B}=380 \mathrm{MHz} \\
\Delta v_{30 d B}=725 \mathrm{MHz}\end{array}$ & $\begin{array}{c}\Delta v=75 \mathrm{MHz} \\
\Delta v_{10 d B}=225 \mathrm{MHz} \\
\Delta v_{20 d B}=746 \mathrm{MHz} \\
\Delta v_{30 \mathrm{~dB}}=2370.5 \mathrm{MHz}\end{array}$ \\
\hline $1530 \mathrm{~nm}$ & $\begin{array}{c}\Delta v=100 \mathrm{MHz} \\
\Delta v_{10 d B}=200 \mathrm{MHz} \\
\Delta v_{20 d B}=459 \mathrm{MHz} \\
\Delta v_{30 d B}=825 \mathrm{MHz}\end{array}$ & $\begin{array}{c}\Delta v=100 \mathrm{MHz} \\
\Delta v_{10 d B}=300 \mathrm{MHz} \\
\Delta v_{20 d B}=995 \mathrm{MHz} \\
\Delta v_{30 \mathrm{~dB}}=3161 \mathrm{MHz}\end{array}$ \\
\hline $1531 \mathrm{~nm}$ & $\begin{array}{c}\Delta v=100 \mathrm{MHz} \\
\Delta v_{10 \mathrm{~dB}}=215 \mathrm{MHz}\end{array}$ & $\begin{array}{c}\Delta v=100 \mathrm{MHz} \\
\Delta v_{10 \mathrm{~dB}}=300 \mathrm{MHz}\end{array}$ \\
\hline
\end{tabular}




\begin{tabular}{|c|c|c|}
\hline & $\begin{array}{l}\Delta v_{20 d B}=440 \mathrm{MHz} \\
\Delta v_{30 d B}=750 \mathrm{MHz}\end{array}$ & $\begin{array}{c}\Delta v_{20 d B}=995 \mathrm{MHz} \\
\Delta v_{30 d B}=3161 \mathrm{MHz}\end{array}$ \\
\hline $1532 \mathrm{~nm}$ & $\begin{array}{c}\Delta v=140 \mathrm{MHz} \\
\Delta v_{10 d B}=300 \mathrm{MHz} \\
\Delta v_{20 d B}=530 \mathrm{MHz} \\
\Delta v_{30 d B}=900 \mathrm{MHz}\end{array}$ & $\begin{array}{c}\Delta v=140 \mathrm{MHz} \\
\Delta v_{10 d B}=420 \mathrm{MHz} \\
\Delta v_{20 d B}=1393 \mathrm{MHz} \\
\Delta v_{30 d B}=4425 \mathrm{MHz}\end{array}$ \\
\hline $1533 \mathrm{~nm}$ & $\begin{array}{c}\Delta v=115 \mathrm{MHz} \\
\Delta v_{10 d B}=230 \mathrm{MHz} \\
\Delta v_{20 d B}=465 \mathrm{MHz} \\
\Delta v_{30 d B}=865 \mathrm{MHz}\end{array}$ & $\begin{array}{c}\Delta v=115 \mathrm{MHz} \\
\Delta v_{10 d B}=345 \mathrm{MHz} \\
\Delta v_{20 d B}=1144 \mathrm{MHz} \\
\Delta v_{30 d B}=3635 \mathrm{MHz}\end{array}$ \\
\hline $1534 \mathrm{~nm}$ & $\begin{array}{c}\Delta v=75 \mathrm{MHz} \\
\Delta v_{10 d B}=160 \mathrm{MHz} \\
\Delta v_{20 d B}=325 \mathrm{MHz} \\
\Delta v_{30 d B}=640 \mathrm{MHz}\end{array}$ & $\begin{array}{c}\Delta v=75 \mathrm{MHz} \\
\Delta v_{10 d B}=225 \mathrm{MHz} \\
\Delta v_{20 d B}=746 \mathrm{MHz} \\
\Delta v_{30 d B}=2370.5 \mathrm{MHz}\end{array}$ \\
\hline $1535 \mathrm{~nm}$ & $\begin{array}{c}\Delta v=80 \mathrm{MHz} \\
\Delta v_{10 d B}=195 \mathrm{MHz} \\
\Delta v_{20 d B}=415 \mathrm{MHz} \\
\Delta v_{30 d B}=850 \mathrm{MHz}\end{array}$ & $\begin{array}{c}\Delta v=80 \mathrm{MHz} \\
\Delta v_{10 d B}=240 \mathrm{MHz} \\
\Delta v_{20 d B}=796 \mathrm{MHz} \\
\Delta v_{30 d B}=2528.6 \mathrm{MHz}\end{array}$ \\
\hline $1536 \mathrm{~nm}$ & $\begin{array}{c}\Delta v=80 \mathrm{MHz} \\
\Delta v_{10 d B}=190 \mathrm{MHz} \\
\Delta v_{20 d B}=400 \mathrm{MHz} \\
\Delta v_{30 d B}=820 \mathrm{MHz}\end{array}$ & $\begin{array}{c}\Delta v=80 \mathrm{MHz} \\
\Delta v_{10 d B}=240 \mathrm{MHz} \\
\Delta v_{20 d B}=796 \mathrm{MHz} \\
\Delta v_{30 d B}=2528.6 \mathrm{MHz}\end{array}$ \\
\hline $1537 \mathrm{~nm}$ & $\begin{array}{c}\Delta v=150 \mathrm{MHz} \\
\Delta v_{10 d B}=270 \mathrm{MHz} \\
\Delta v_{20 d B}=550 \mathrm{MHz} \\
\Delta v_{30 d B}=1000 \mathrm{MHz}\end{array}$ & $\begin{array}{c}\Delta v=150 \mathrm{MHz} \\
\Delta v_{10 d B}=450 \mathrm{MHz} \\
\Delta v_{20 d B}=1492.5 \mathrm{MHz} \\
\Delta v_{30 d B}=4741 \mathrm{MHz}\end{array}$ \\
\hline $1538 \mathrm{~nm}$ & $\begin{array}{c}\Delta v=125 \mathrm{MHz} \\
\Delta v_{10 \mathrm{~dB}}=250 \mathrm{MHz} \\
\Delta v_{20 \mathrm{~dB}}=515 \mathrm{MHz} \\
\Delta v_{30 \mathrm{~dB}}=940 \mathrm{MHz}\end{array}$ & $\begin{array}{c}\Delta v=125 \mathrm{MHz} \\
\Delta v_{10 d B}=375 \mathrm{MHz} \\
\Delta v_{20 d B}=1243.7 \mathrm{MHz} \\
\Delta v_{30 d B}=3951 \mathrm{MHz}\end{array}$ \\
\hline $1539 \mathrm{~nm}$ & $\begin{array}{c}\Delta v=50 \mathrm{MHz} \\
\Delta v_{10 d B}=135 \mathrm{MHz} \\
\Delta v_{20 d B}=310 \mathrm{MHz} \\
\Delta v_{30 d B}=620 \mathrm{MHz}\end{array}$ & $\begin{array}{c}\Delta v=50 \mathrm{MHz} \\
\Delta v_{10 d B}=150 \mathrm{MHz} \\
\Delta v_{20 d B}=497.5 \mathrm{MHz} \\
\Delta v_{30 d B}=1580 \mathrm{MHz}\end{array}$ \\
\hline $1540 \mathrm{~nm}$ & $\begin{array}{c}\Delta v=55 \mathrm{MHz} \\
\Delta v_{10 d B}=175 \mathrm{MHz} \\
\Delta v_{20 d B}=375 \mathrm{MHz} \\
\Delta v_{30 d B}=890 \mathrm{MHz}\end{array}$ & $\begin{array}{c}\Delta v=55 \mathrm{MHz} \\
\Delta v_{10 d B}=165 \mathrm{MHz} \\
\Delta v_{20 d B}=796 \mathrm{MHz} \\
\Delta v_{30 d B}=2528.6 \mathrm{MHz}\end{array}$ \\
\hline
\end{tabular}




\begin{tabular}{|c|c|c|}
\hline $1541 \mathrm{~nm}$ & $\begin{array}{c}\Delta v=80 \mathrm{MHz} \\
\Delta v_{10 \mathrm{~dB}}=190 \mathrm{MHz} \\
\Delta v_{20 \mathrm{~dB}}=400 \mathrm{MHz} \\
\Delta v_{30 \mathrm{~dB}}=895 \mathrm{MHz}\end{array}$ & $\begin{array}{c}\Delta v=80 \mathrm{MHz} \\
\Delta v_{10 d B}=240 \mathrm{MHz} \\
\Delta v_{20 d B}=796 \mathrm{MHz} \\
\Delta v_{30 d B}=2528.6 \mathrm{MHz}\end{array}$ \\
\hline $1542 \mathrm{~nm}$ & $\begin{array}{c}\Delta v=90 \mathrm{MHz} \\
\Delta v_{10 \mathrm{~dB}}=215 \mathrm{MHz} \\
\Delta v_{20 \mathrm{~dB}}=440 \mathrm{MHz} \\
\Delta v_{30 \mathrm{~dB}}=930 \mathrm{MHz}\end{array}$ & $\begin{array}{c}\Delta v=90 \mathrm{MHz} \\
\Delta v_{10 d B}=270 \mathrm{MHz} \\
\Delta v_{20 d B}=895.5 \mathrm{MHz} \\
\Delta v_{30 d B}=2844.6 \mathrm{MHz}\end{array}$ \\
\hline $1543 \mathrm{~nm}$ & $\begin{array}{c}\Delta v=115 \mathrm{MHz} \\
\Delta v_{10 d B}=250 \mathrm{MHz} \\
\Delta v_{20 d B}=510 \mathrm{MHz} \\
\Delta v_{30 \mathrm{~dB}}=>1000 \mathrm{MHz}\end{array}$ & $\begin{array}{c}\Delta v=115 \mathrm{MHz} \\
\Delta v_{10 d B}=345 \mathrm{MHz} \\
\Delta v_{20 d B}=1144 \mathrm{MHz} \\
\Delta v_{30 d B}=3635 \mathrm{MHz}\end{array}$ \\
\hline $1544 \mathrm{~nm}$ & $\begin{array}{c}\Delta v=140 \mathrm{MHz} \\
\Delta v_{10 \mathrm{~dB}}=315 \mathrm{MHz} \\
\Delta v_{20 \mathrm{~dB}}=590 \mathrm{MHz} \\
\Delta v_{30 \mathrm{~dB}}=>1000 \mathrm{MHz}\end{array}$ & $\begin{array}{c}\Delta v=140 \mathrm{MHz} \\
\Delta v_{10 d B}=420 \mathrm{MHz} \\
\Delta v_{20 d B}=1393 \mathrm{MHz} \\
\Delta v_{30 d B}=4425 \mathrm{MHz}\end{array}$ \\
\hline $1545 \mathrm{~nm}$ & $\begin{array}{c}\Delta v=50 \mathrm{MHz} \\
\Delta v_{10 \mathrm{~dB}}=130 \mathrm{MHz} \\
\Delta v_{20 \mathrm{~dB}}=325 \mathrm{MHz} \\
\Delta v_{30 \mathrm{~dB}}=880 \mathrm{MHz}\end{array}$ & $\begin{array}{c}\Delta v=50 \mathrm{MHz} \\
\Delta v_{10 d B}=150 \mathrm{MHz} \\
\Delta v_{20 d B}=497.5 \mathrm{MHz} \\
\Delta v_{30 d B}=1580 \mathrm{MHz}\end{array}$ \\
\hline $1546 \mathrm{~nm}$ & $\begin{array}{c}\Delta v=50 \mathrm{MHz} \\
\Delta v_{10 \mathrm{~dB}}=130 \mathrm{MHz} \\
\Delta v_{20 \mathrm{~dB}}=320 \mathrm{MHz} \\
\Delta v_{30 \mathrm{~dB}}=885 \mathrm{MHz}\end{array}$ & $\begin{array}{c}\Delta v=50 \mathrm{MHz} \\
\Delta v_{10 d B}=150 \mathrm{MHz} \\
\Delta v_{20 d B}=497.5 \mathrm{MHz} \\
\Delta v_{30 d B}=1580 \mathrm{MHz}\end{array}$ \\
\hline $1547 \mathrm{~nm}$ & $\begin{array}{c}\Delta v=80 \mathrm{MHz} \\
\Delta v_{10 \mathrm{~dB}}=215 \mathrm{MHz} \\
\Delta v_{20 \mathrm{~dB}}=450 \mathrm{MHz} \\
\Delta v_{30 \mathrm{~dB}}=1000 \mathrm{MHz}\end{array}$ & $\begin{array}{c}\Delta v=80 \mathrm{MHz} \\
\Delta v_{10 d B}=240 \mathrm{MHz} \\
\Delta v_{20 d B}=796 \mathrm{MHz} \\
\Delta v_{30 d B}=2528.6 \mathrm{MHz}\end{array}$ \\
\hline $1548 \mathrm{~nm}$ & $\begin{array}{c}\Delta v=100 \mathrm{MHz} \\
\Delta v_{10 \mathrm{~dB}}=215 \mathrm{MHz} \\
\Delta v_{20 \mathrm{~dB}}=450 \mathrm{MHz} \\
\Delta v_{30 \mathrm{~dB}}=1000 \mathrm{MHz}\end{array}$ & $\begin{array}{c}\Delta v=100 \mathrm{MHz} \\
\Delta v_{10 d B}=300 \mathrm{MHz} \\
\Delta v_{20 d B}=995 \mathrm{MHz} \\
\Delta v_{30 d B}=3160.7 \mathrm{MHz}\end{array}$ \\
\hline $1549 \mathrm{~nm}$ & $\begin{array}{c}\Delta v=145 \mathrm{MHz} \\
\Delta v_{10 \mathrm{~dB}}=250 \mathrm{MHz} \\
\Delta v_{20 \mathrm{~dB}}=515 \mathrm{MHz} \\
\Delta v_{30 \mathrm{~dB}}=>1000 \mathrm{MHz}\end{array}$ & $\begin{array}{c}\Delta v=145 \mathrm{MHz} \\
\Delta v_{10 d B}=435 \mathrm{MHz} \\
\Delta v_{20 d B}=1442.7 \mathrm{MHz} \\
\Delta v_{30 d B}=4583 \mathrm{MHz}\end{array}$ \\
\hline
\end{tabular}




\begin{tabular}{|c|c|c|}
\hline $1550 \mathrm{~nm}$ & $\begin{array}{c}\Delta v=145 \mathrm{MHz} \\
\Delta v_{10 \mathrm{~dB}}=310 \mathrm{MHz} \\
\Delta v_{20 \mathrm{~dB}}=610 \mathrm{MHz} \\
\Delta v_{30 \mathrm{~dB}}=>1000 \mathrm{MHz}\end{array}$ & $\begin{array}{c}\Delta v=145 \mathrm{MHz} \\
\Delta v_{10 \mathrm{~dB}}=435 \mathrm{MHz} \\
\Delta v_{20 d B}=1442.7 \mathrm{MHz} \\
\Delta v_{30 \mathrm{~dB}}=4583 \mathrm{MHz}\end{array}$ \\
\hline $1551 \mathrm{~nm}$ & $\begin{array}{c}\Delta v=60 \mathrm{MHz} \\
\Delta v_{10 d B}=150 \mathrm{MHz} \\
\Delta v_{20 d B}=340 \mathrm{MHz} \\
\Delta v_{30 d B}=920 \mathrm{MHz}\end{array}$ & $\begin{array}{c}\Delta v=60 \mathrm{MHz} \\
\Delta v_{10 d B}=180 \mathrm{MHz} \\
\Delta v_{20 d B}=597 \mathrm{MHz} \\
\Delta v_{30 d B}=1896.4 \mathrm{MHz}\end{array}$ \\
\hline $1552 \mathrm{~nm}$ & $\begin{array}{c}\Delta v=80 \mathrm{MHz} \\
\Delta v_{10 \mathrm{~dB}}=185 \mathrm{MHz} \\
\Delta v_{20 \mathrm{~dB}}=400 \mathrm{MHz} \\
\Delta v_{30 \mathrm{~dB}}=1000 \mathrm{MHz}\end{array}$ & $\begin{array}{c}\Delta v=80 \mathrm{MHz} \\
\Delta v_{10 d B}=240 \mathrm{MHz} \\
\Delta v_{20 d B}=796 \mathrm{MHz} \\
\Delta v_{30 d B}=2528.6 \mathrm{MHz}\end{array}$ \\
\hline $1553 \mathrm{~nm}$ & $\begin{array}{c}\Delta v=100 \mathrm{MHz} \\
\Delta v_{10 \mathrm{~dB}}=220 \mathrm{MHz} \\
\Delta v_{20 \mathrm{~dB}}=500 \mathrm{MHz} \\
\Delta v_{30 \mathrm{~dB}}=>1000 \mathrm{MHz}\end{array}$ & $\begin{array}{c}\Delta v=100 \mathrm{MHz} \\
\Delta v_{10 \mathrm{~dB}}=300 \mathrm{MHz} \\
\Delta v_{20 \mathrm{~dB}}=995 \mathrm{MHz} \\
\Delta v_{30 d B}=3160.7 \mathrm{MHz}\end{array}$ \\
\hline $1554 \mathrm{~nm}$ & $\begin{array}{c}\Delta v=115 \mathrm{MHz} \\
\Delta v_{10 d B}=250 \mathrm{MHz} \\
\Delta v_{20 \mathrm{~dB}}=540 \mathrm{MHz} \\
\Delta v_{30 \mathrm{~dB}}=>1000 \mathrm{MHz}\end{array}$ & $\begin{array}{c}\Delta v=115 \mathrm{MHz} \\
\Delta v_{10 d B}=345 \mathrm{MHz} \\
\Delta v_{20 d B}=1144 \mathrm{MHz} \\
\Delta v_{30 d B}=3635 \mathrm{MHz}\end{array}$ \\
\hline $1555 \mathrm{~nm}$ & $\begin{array}{c}\Delta v=110 \mathrm{MHz} \\
\Delta v_{10 \mathrm{~dB}}=225 \mathrm{MHz} \\
\Delta v_{20 \mathrm{~dB}}=450 \mathrm{MHz} \\
\Delta v_{30 \mathrm{~dB}}=>1000 \mathrm{MHz}\end{array}$ & $\begin{array}{c}\Delta v=110 \mathrm{MHz} \\
\Delta v_{10 \mathrm{~dB}}=330 \mathrm{MHz} \\
\Delta v_{20 d B}=1094.5 \mathrm{MHz} \\
\Delta v_{30 d B}=3476.8 \mathrm{MHz}\end{array}$ \\
\hline $1557 \mathrm{~nm}$ & $\begin{array}{c}\Delta v=65 \mathrm{MHz} \\
\Delta v_{10 d B}=165 \mathrm{MHz} \\
\Delta v_{20 d B}=365 \mathrm{MHz} \\
\Delta v_{30 d B}=990 \mathrm{MHz}\end{array}$ & $\begin{array}{c}\Delta v=65 \mathrm{MHz} \\
\Delta v_{10 d B}=195 \mathrm{MHz} \\
\Delta v_{20 d B}=646.7 \mathrm{MHz} \\
\Delta v_{30 d B}=2054.5 \mathrm{MHz}\end{array}$ \\
\hline $1558 \mathrm{~nm}$ & $\begin{array}{c}\Delta v=95 \mathrm{MHz} \\
\Delta v_{10 d B}=215 \mathrm{MHz} \\
\Delta v_{20 d B}=485 \mathrm{MHz} \\
\Delta v_{30 d B}=>1000 \mathrm{MHz}\end{array}$ & $\begin{array}{c}\Delta v=95 \mathrm{MHz} \\
\Delta v_{10 d B}=285 \mathrm{MHz} \\
\Delta v_{20 d B}=945 \mathrm{MHz} \\
\Delta v_{30 d B}=3002.7 \mathrm{MHz}\end{array}$ \\
\hline $1559 \mathrm{~nm}$ & $\begin{array}{c}\Delta v=95 \mathrm{MHz} \\
\Delta v_{10 d B}=225 \mathrm{MHz} \\
\Delta v_{20 d B}=510 \mathrm{MHz} \\
\Delta v_{30 d B}=>1000 \mathrm{MHz}\end{array}$ & $\begin{array}{c}\Delta v=95 \mathrm{MHz} \\
\Delta v_{10 d B}=285 \mathrm{MHz} \\
\Delta v_{20 d B}=945 \mathrm{MHz} \\
\Delta v_{30 d B}=3002.7 \mathrm{MHz}\end{array}$ \\
\hline $1560 \mathrm{~nm}$ & $\begin{array}{c}\Delta v=95 \mathrm{MHz} \\
\Delta v_{10 d B}=230 \mathrm{MHz}\end{array}$ & $\begin{array}{c}\Delta v=95 \mathrm{MHz} \\
\Delta v_{10 \mathrm{~dB}}=285 \mathrm{MHz}\end{array}$ \\
\hline
\end{tabular}




\begin{tabular}{|c|c|c|}
\hline & $\begin{array}{c}\Delta v_{20 d B}=490 \mathrm{MHz} \\
\Delta v_{30 d B}=>1000 \mathrm{MHz}\end{array}$ & $\begin{array}{c}\Delta v_{20 \mathrm{~dB}}=945 \mathrm{MHz} \\
\Delta v_{30 d B}=3002.7 \mathrm{MHz}\end{array}$ \\
\hline $1562 \mathrm{~mm}$ & $\Delta v=300 \mathrm{MHz}$ & $\Delta v=300 \mathrm{MHz}$ \\
& $\Delta v_{10 d B}=550 \mathrm{MHz}$ & $\Delta v_{10 d B}=900 \mathrm{MHz}$ \\
& $\Delta v_{20 d B}=>1000 \mathrm{MHz}$ & $\Delta v_{20 d B}=2985 \mathrm{MHz}$ \\
& $\Delta v_{30 d B}=>1000 \mathrm{MHz}$ & $\Delta v_{30 d B}=9482 \mathrm{MHz}$ \\
\hline \multirow{2}{*}{$1563 \mathrm{~nm}$} & $\Delta v=290 \mathrm{MHz}$ & $\Delta v=290 \mathrm{MHz}$ \\
& $\Delta v_{10 \mathrm{~dB}}=585 \mathrm{MHz}$ & $\Delta v_{10 \mathrm{~dB}}=870 \mathrm{MHz}$ \\
& $\Delta v_{20 d B}=>1000 \mathrm{MHz}$ & $\Delta v_{20 d B}=2885.5 \mathrm{MHz}$ \\
& $\Delta v_{30 d B}=>1000 \mathrm{MHz}$ & $\Delta v_{30 d B}=9166 \mathrm{MHz}$ \\
\hline
\end{tabular}

\begin{tabular}{|c|c|c|}
\hline $1564 \mathrm{~nm}$ & $\Delta v=100 \mathrm{MHz}$ & $\Delta v=100 \mathrm{MHz}$ \\
& $\Delta v_{10 d B}=230 \mathrm{MHz}$ & $\Delta v_{10 d B}=300 \mathrm{MHz}$ \\
& $\Delta v_{20 d B}=460 \mathrm{MHz}$ & $\Delta v_{20 d B}=995 \mathrm{MHz}$ \\
& $\Delta v_{30 d B}=>1000 \mathrm{MHz}$ & $\Delta v_{30 d B}=3160.7 \mathrm{MHz}$ \\
\hline $1565 \mathrm{~nm}$ & $\Delta v=125 \mathrm{MHz}$ & $\Delta v=125 \mathrm{MHz}$ \\
& $\Delta v_{10 d B}=260 \mathrm{MHz}$ & $\Delta v_{10 d B}=375 \mathrm{MHz}$ \\
& $\Delta v_{20 d B}=930 \mathrm{MHz}$ & $\Delta v_{20 d B}=1243.7 \mathrm{MHz}$ \\
& $\Delta v_{30 d B}=>1000 \mathrm{MHz}$ & $\Delta v_{30 d B}=3951 \mathrm{MHz}$ \\
\hline $1566 \mathrm{~nm}$ & $\Delta v=120 \mathrm{MHz}$ & $\Delta v=120 \mathrm{MHz}$ \\
& $\Delta v_{10 d B}=260 \mathrm{MHz}$ & $\Delta v_{10 d B}=360 \mathrm{MHz}$ \\
& $\Delta v_{20 d B}=600 \mathrm{MHz}$ & $\Delta v_{20 d B}=1194 \mathrm{MHz}$ \\
& $\Delta v_{30 d B}=>1000 \mathrm{MHz}$ & $\Delta v_{30 d B}=3793 \mathrm{MHz}$ \\
\hline
\end{tabular}




\section{Appendix C. Time Domain Interference Fringes}

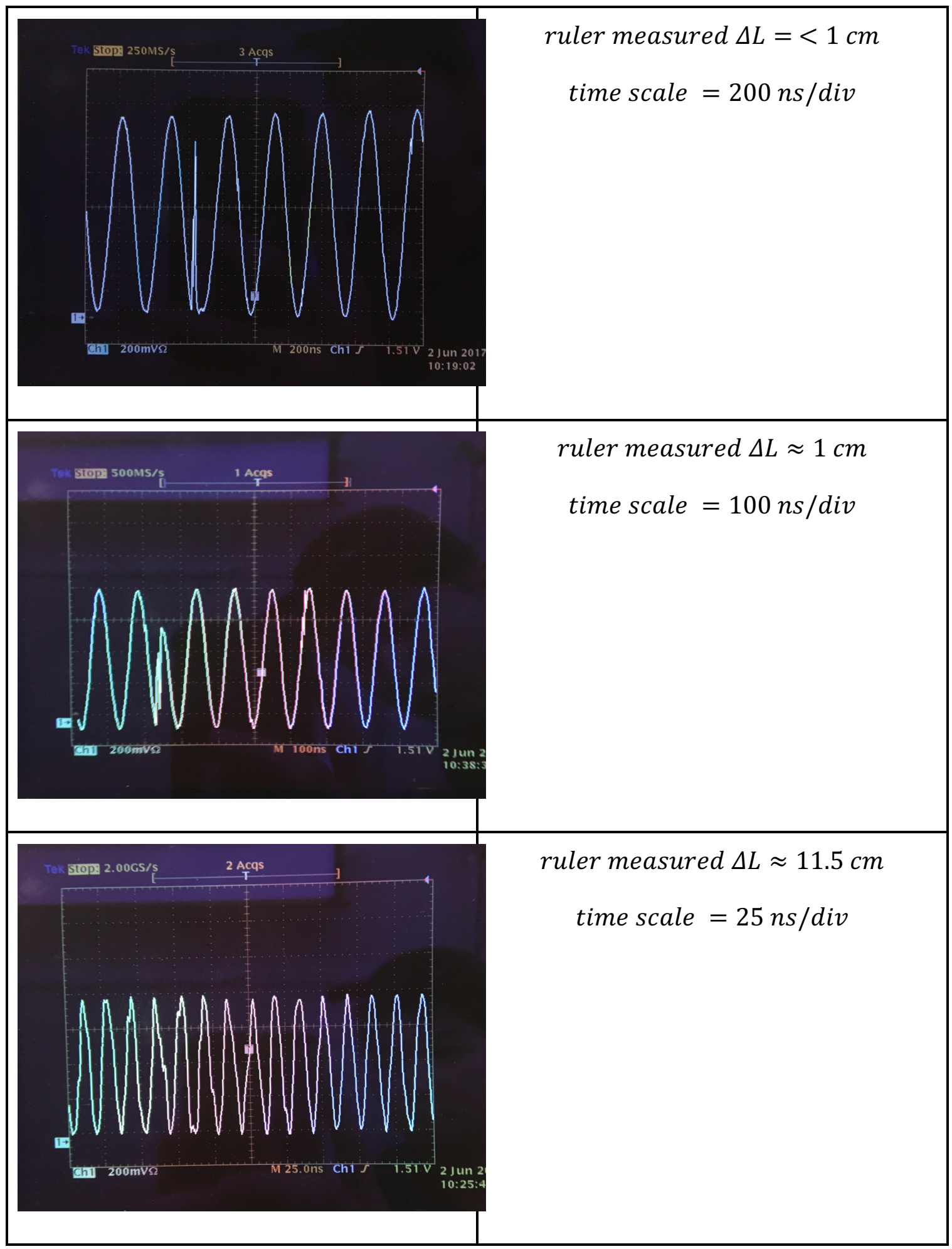




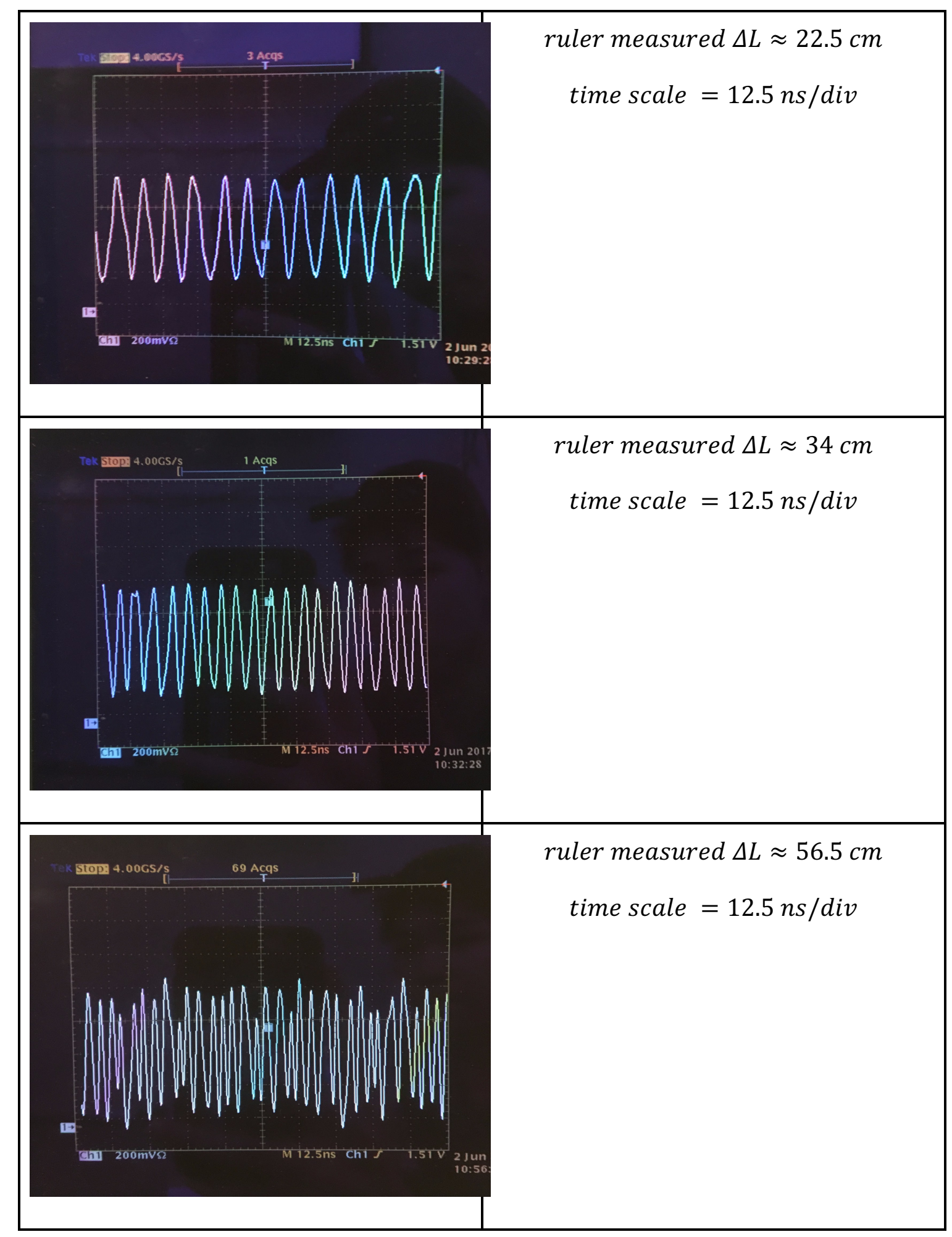




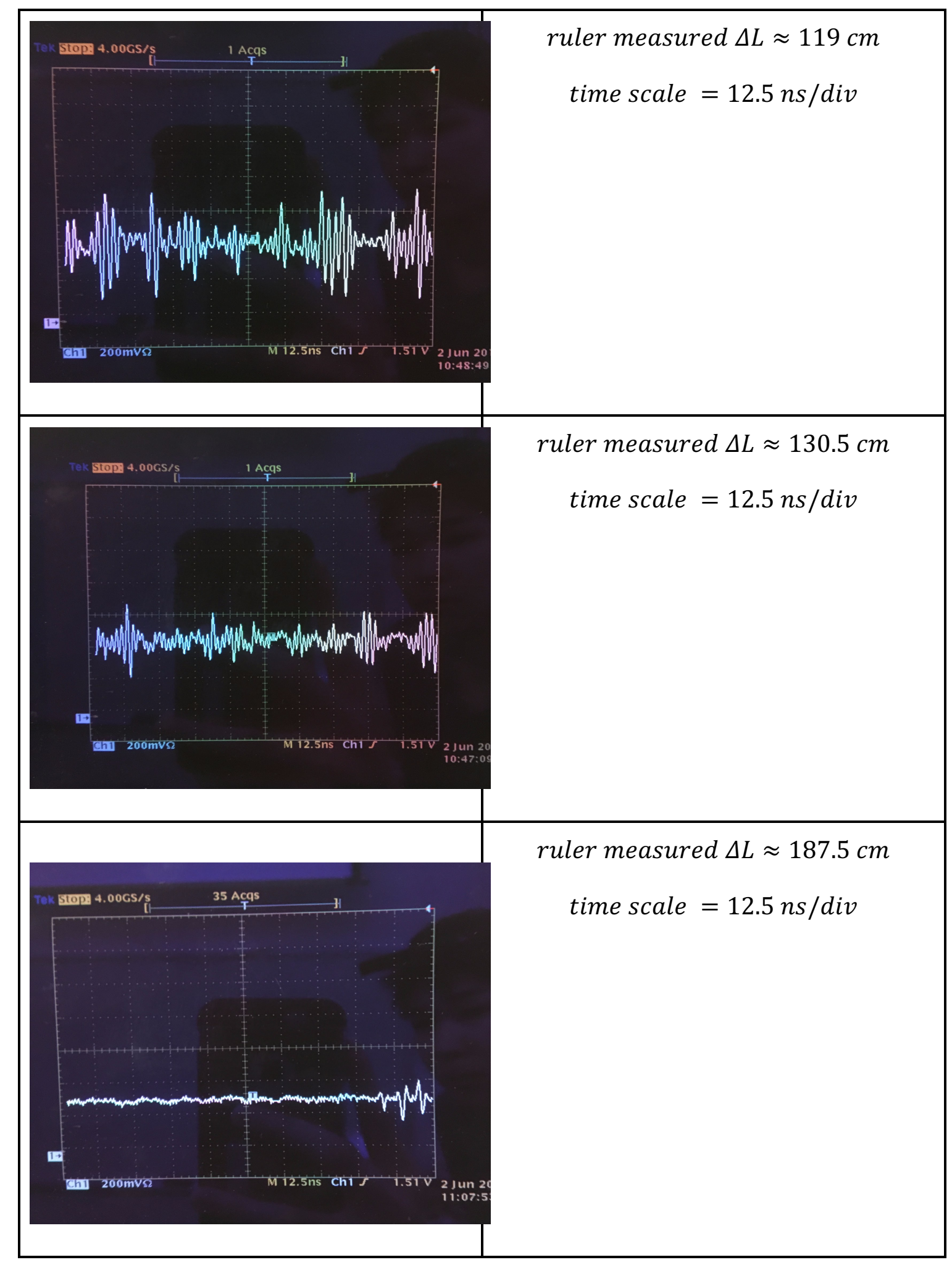




\section{Appendix D. Frequency Domain Interference Fringes}

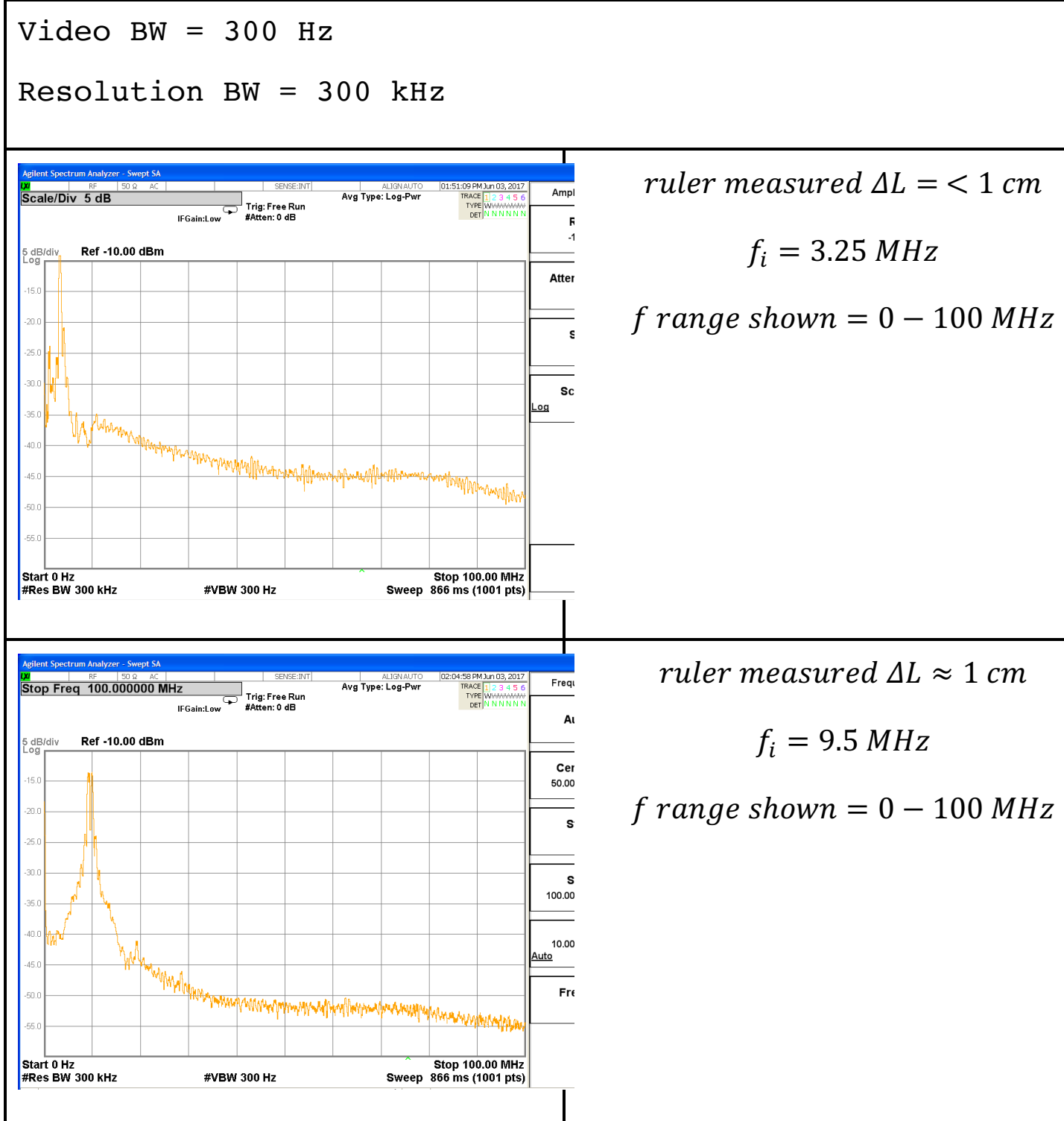




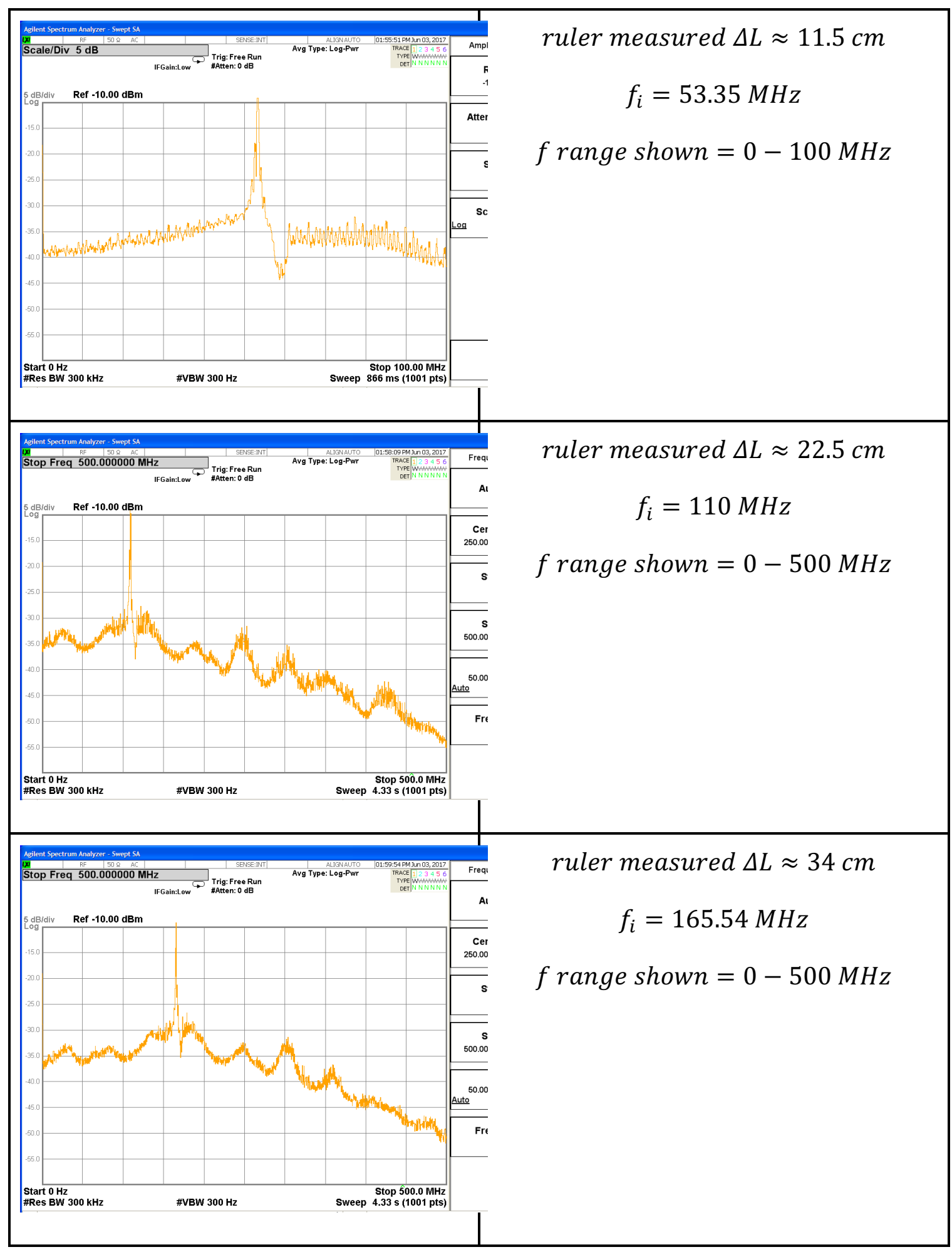




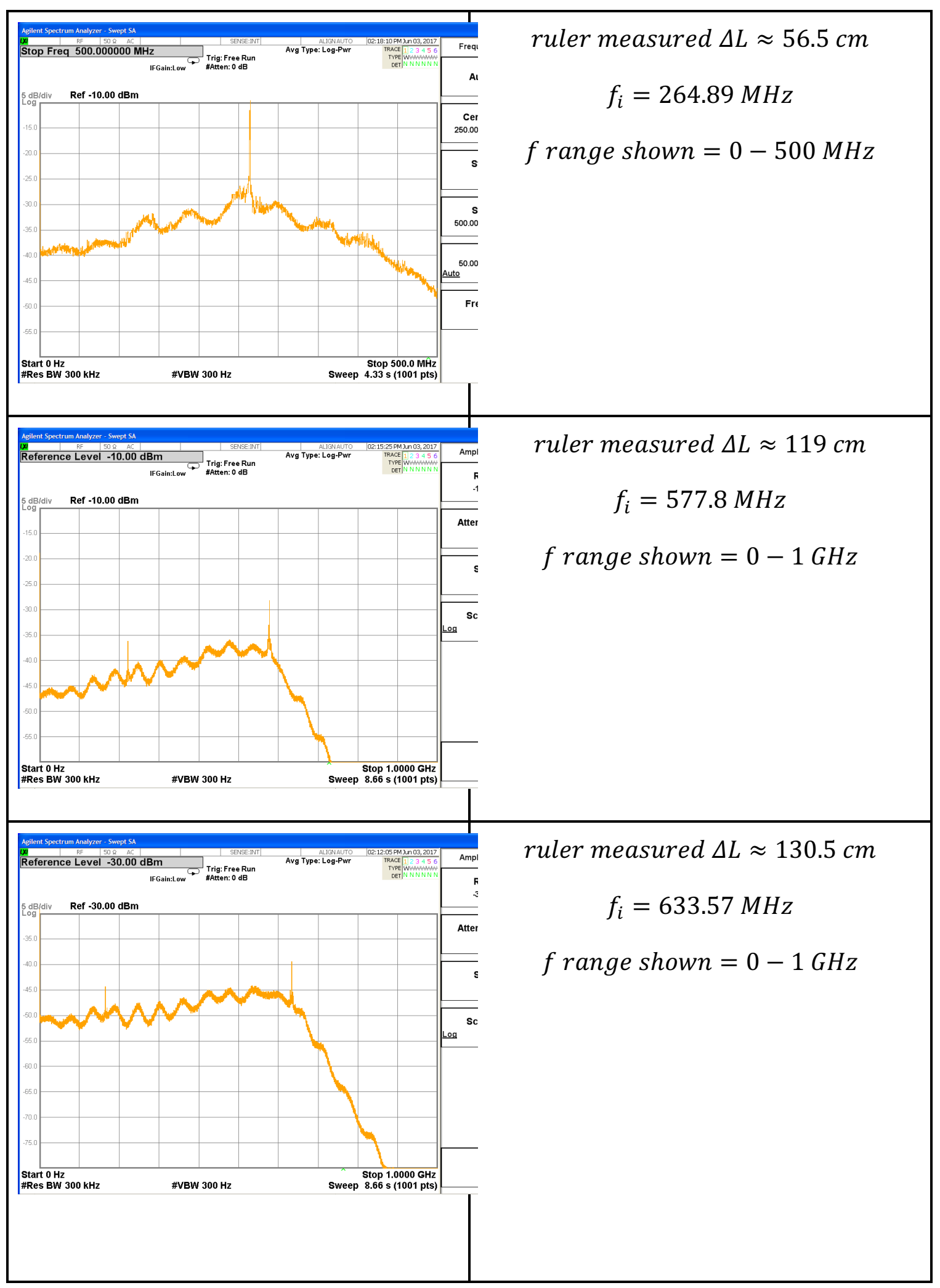




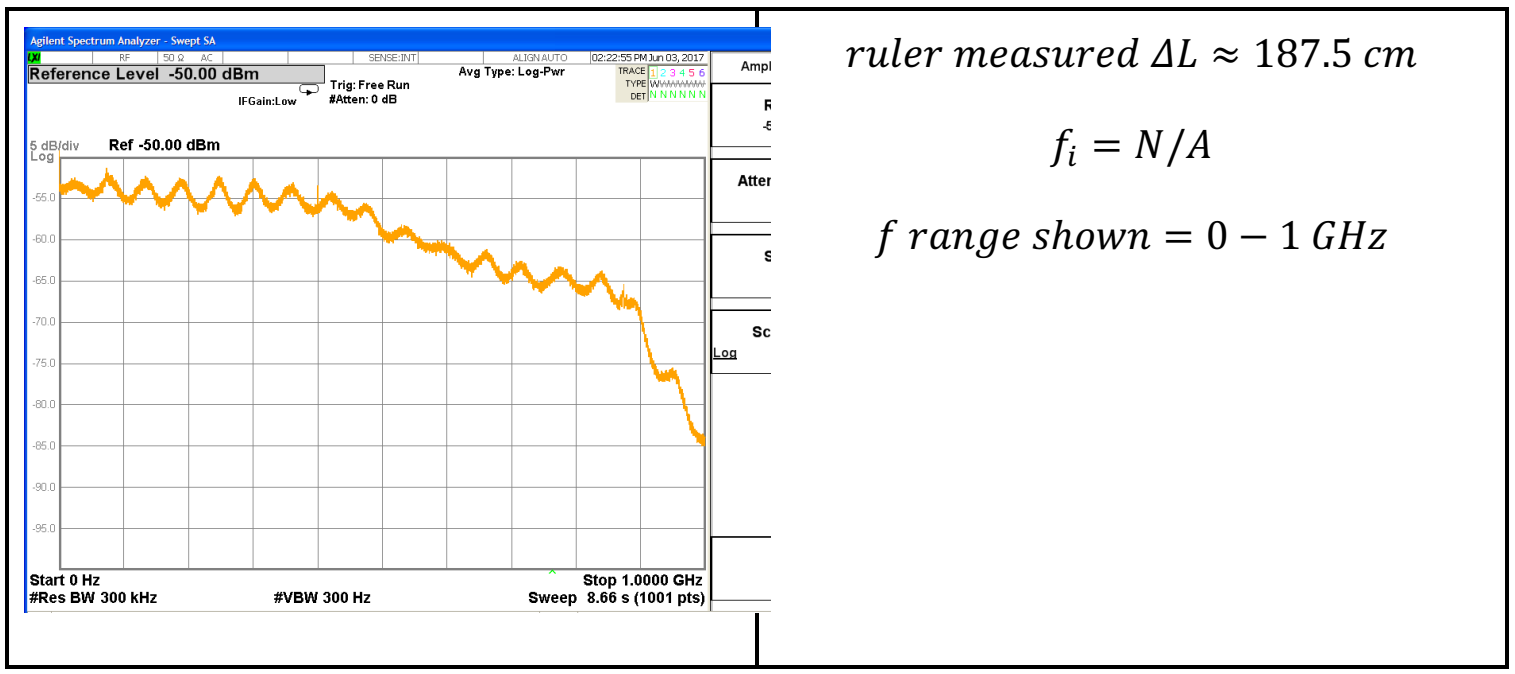

